\title{
Evaluating totipotency using criteria of increasing stringency
}

3 Eszter Posfai ${ }^{\mathrm{a}, \mathrm{b}, \#, \star}$ John Paul Schell ${ }^{\mathrm{c}, \mathrm{d}, \#}$, Adrian Janiszewski $^{\mathrm{f}, \#}$, Isidora Rovic $^{\mathrm{e}}$, Alexander Murray $^{\mathrm{a}}$,

4 Brian Bradshaw ${ }^{a}$, Tatsuya Yamakawa ${ }^{a}$, Tine Pardon ${ }^{f}$, Mouna El Bakkali $^{f}$, Irene Talon $^{f}$, Natalie

5 De Geest ${ }^{\mathrm{f}}$, Pankaj Kumar ${ }^{\mathrm{c}, \mathrm{d}}$, San Kit To ${ }^{\mathrm{f}}$, Sophie Petropoulos ${ }^{\mathrm{c}, \mathrm{d}, \mathrm{g}}$, Andrea Jurisicova ${ }^{\mathrm{e}, \mathrm{h}}$, Vincent

6 Pasque ${ }^{\mathrm{f}, *}$, Fredrik Lanner ${ }^{\mathrm{c}, \mathrm{d}, \mathrm{i} *}$, Janet Rossant ${ }^{\mathrm{a}, *}$

8 aprogram in Developmental and Stem Cell Biology, Hospital for Sick Children, Toronto, ON,

9 Canada

10 bCurrent: Department of Molecular Biology, Princeton University, Princeton, NJ, USA

11 'Department of Clinical Sciences, Intervention and Technology, Karolinska Institutet, Stockholm,

12 Sweden

13 Division of Obstetrics and Gynecology, Karolinska Universitetssjukhuset, Stockholm, Sweden.

14 eLunenfeld Tanenbaum Research Institute, Sinai Health System, Toronto, ON, Canada

15 fKU Leuven-University of Leuven, Department of Development and Regeneration, Leuven Stem

16 Cell Institute, Leuven, Belgium

$17{ }^{9}$ Centre de recherche du centre hospitalier de l'université de montréal (CRCHUM), University of

18 Montreal, Department of Medicine, Montreal, QC, Canada

19 hDepartments of Obstetrics and Gynecology and Department of Physiology, Toronto, ON,

20 Canada

21 'Ming Wai Lau Center for Reparative Medicine, Stockholm node, Karolinska Institutet,

22 Stockholm, Sweden

24 \#authors contributed equally

25 *corresponding, co-last authors. 


\section{Abstract}

2 Totipotency is the ability of a single cell to give rise to all the differentiated cell types that build the

3 conceptus, yet how to capture this property in vitro remains incompletely understood. Defining

4 totipotency relies upon a variety of assays of variable stringency. Here we describe criteria to

5 define totipotency. We illustrate how distinct criteria of increasing stringency can be used to judge

6 totipotency by evaluating candidate totipotent cell types in the mouse, including early blastomeres

7 and expanded or extended pluripotent stem cells. Our data challenge the notion that expanded

8 or extended pluripotent states harbor increased totipotent potential relative to conventional

9 embryonic stem cells under in vivo conditions.

\section{Introduction}

12 Totipotency in mammals is limited to early-stage blastomeres. In its most stringent definition,

13 totipotency is the ability of a single cell to give rise to an entire embryo and its extra-embryonic

14 components - which in mouse is limited to the 1 - and the 2 -cell stages of development ${ }^{1}$. By a

15 less-stringent definition, a cell is considered totipotent if it has an unbiased ability to contribute to

16 both embryonic and extra-embryonic lineages, and is lost as cells gradually restrict their

17 developmental potential and commit to distinct cell lineages by the blastocyst stage ${ }^{2,3,4}$. In mouse,

18 the first cell fate decision starting at embryonic day (E)2.5 sets aside the trophectoderm (TE), the

19 precursors of the placenta, from the inner cell mass (ICM). A second cell fate decision starting

20 around E3.5 within the ICM gives rise to the pluripotent epiblast (EPI) and the primitive endoderm

21 (PE), precursors of all embryonic germ layers and extra-embryonic yolk sac, respectively ${ }^{5}$. For

22 the most part, EPI, PE and TE cells maintain lineage assignments throughout subsequent

23 development, with a notable exception for the PE, which was shown to also contribute to EPI-

24 derived definitive endodermal lineages during post-implantation ${ }^{6,7}$. 
1 While EPI, PE and TE cells exist only transiently in the developing embryo, distinct self-renewing

2 stem cell types can be derived from each cell type using a combination of appropriate growth

3 factors and/or inhibitors which capture and preserve their developmental potential in culture $8,9,10,11$.

4 Murine embryonic stem cells (ESCs) derived from the early EPI lineage were originally

5 established using fetal bovine serum and leukemia inhibitory factor (Lif) ${ }^{8,9}$. They can, however,

6 also be cultured under naïve conditions, using inhibitors against mitogen-activated protein kinase

7 and glycogen synthase kinase-3, termed $2 \mathrm{i}$, in combination with Lif ${ }^{12}$. Trophoblast stem cells

8 (TSCs) can be derived from the TE lineage using fibroblast growth factor $4^{10}$ and extra-embryonic

9 endoderm (XEN) stem cells can be established from the PE using various methods ${ }^{11,13,14}$.

10 Importantly, while each of these stem cell types are able to re-enter the normal course of

11 embryonic development and differentiate into the downstream cell types similar to their in vivo

12 counterparts, they are also lineage-restricted in that they do not readily cross lineage boundaries

13 that have been set during blastocyst formation ${ }^{15,16}$.

15 Strict lineage restriction differs between the three stem cell types. The closer relationship between

16 EPI and PE lineages is underscored by the observation that ESCs can spontaneously give rise

17 to XEN cells in culture or using soluble factors ${ }^{14,17,18}$. In vivo, ESCs have only been reported to 18 rarely contribute to trophectoderm-derived lineages ${ }^{19}$. Interestingly, 2-cell like cells, which

19 resemble 2-cell blastomeres, transiently emerge from ESC cultures, yet cannot be isolated as

20 stable stem cell lines ${ }^{20,21}$. Work by several laboratories has also shown that stem cell types with

21 properties of $\mathrm{TE}, \mathrm{EPI}$, or $\mathrm{PE}$ can be obtained by reprogramming lineage restriction using

22 transcription factor (TF) expression. Long-term TF overexpression was shown to reprogram ESCs

23 into TSC-like cells in vitro $22,23,24,25$. Induced pluripotent stem cells (iPSCs), as well as induced

24 TSCs and induced XEN stem cells, have also been derived by TF overexpression followed by

25 culture with the appropriate growth media ${ }^{26,27}$. Additionally, mouse primed epiblast stem cells

26 (EpiSCs), isolated from the post-implantation epiblast ${ }^{28,29}$, can be reverted into ESCs $^{30,31}$. 
1 Collectively, these studies suggested that it might be possible to induce totipotent stem cells by

2 conversion from pluripotent stem cells (PSCs).

4 Several conditions have been reported to derive mouse stem cells types with the ability to produce

5 descendants contributing to all three blastocyst-defined lineages $20,32,33,34,35,36,37$. In particular,

6 methods were described to derive extended or expanded PSCs (EPSCs) by conversion from pre-

7 existing ESCs or directly from 8-cell stage blastomeres ${ }^{36,35,38}$. Specifically, Liu lab EPSCs (L-

8 EPSCs) and Deng lab EPSCs (D-EPSCs) were derived in Lif and either 6 or 3 small molecules,

9 respectivelly ${ }^{35,36}$. Both cell types showed molecular and functional features that suggested

10 expanded pluripotency, such as totipotency-associated marker gene expression and contribution

11 to the EPI, PE as well as TE lineages using chimeric assays. Additionally, recent studies reported

12 the ability of EPSCs, alone or in combination with TSCs, to self-assemble into blastocyst-like

13 structures, termed blastoids, that contain cells with features of all three embryonic lineages ${ }^{39,40}$.

14 These studies suggested that stem cells, with the potential to give rise to both ICM and TE

15 lineages - properties that define totipotent stem cells - can be isolated and expanded in vitro.

17 Many criteria of variable stringency can be used to assess totipotency. One criterion is to assess

18 gene expression in search of totipotency-associated marker genes. This can either be performed

19 in bulk for a set of genes or through a more stringent approach taking advantage of transcriptome-

20 wide single-cell correlation analysis with totipotent cells of early embryos. More demanding is

21 providing evidence of the potential to enter both the embryonic and extra-embryonic pathways

22 using in vitro differentiation assays. Finally, a more stringent requirement for evaluating the

23 potential of different stem cell types is to perform in vivo integration experiments, by combining

24 candidate cells with a host embryo and analyzing lineage contributions in the resulting chimera.

25 It is essential to analyze chimeric contributions not only based on localization, but also by 26 assessing lineage integration using functional marker analysis. 
2 Here we subject candidate totipotent stem cells to these assays of increasing stringency to assess

3 their developmental potential. We analyze the transcriptome and gene regulatory networks of

4 ESCs, EPSCs and pre-implantation embryos using bulk and single-cell RNA-sequencing (RNA-

5 seq), and provide a resource for the community enabling interactive online data exploration. We

6 investigate the ability of EPSCs to give rise to TSCs in both a conversion and a reprogramming

7 setting. We analyze the transcriptome and gene regulatory networks of blastoids derived from

8 EPSCs. Finally, we examine how EPSCs and blastomeres perform in chimeric experiments. We

9 present a gold standard for analyzing contribution to different lineages, with a focus on

10 contribution to the trophoblast lineage at different stages combined with molecular analyses. We

11 emphasize the importance of a thorough analysis of cell potential using high stringency assays

12 and highlight ongoing challenges in unlocking the totipotent state.

\section{Results}

17 Transcriptional signatures of pre-implantation embryos and different stem cell states

19 Transcriptomic analyses can serve as effective means to monitor cellular states and analyze

20 marker gene expression. Therefore, we compared the transcriptome of EPSCs, ESCs and 21 embryos. First, we converted ESCs (2iLif) to L-EPSCs and D-EPSCs using published 22 protocols ${ }^{36,35}$. We observed similar morphological changes after conversion as previously 23 reported $^{36,35}$ and were able to stably maintain EPSC lines (Extended Data Fig. 1a). We first used

24 bulk RNA sequencing and set out to explore the dynamics with which a transcriptome shift is 25 induced after switching ESCs into L-EPSC conditions (Fig. 1a). Most changes in gene expression 26 take place within the first 5 days of induction, especially between day 3 and 5 of conversion (Fig. 27 1b, Extended data 2a, Supplementary Tables 1-3). Intriguingly, despite these differences between 
L-EPSCs and ESCs, the L-EPSC transcriptome resembled the ESC transcriptome more than any early mouse embryo stages (Fig. 1c and Extended Data 2b,c) and 4-cell stage embryo marker genes were expressed in a similar pattern in EPSCs and ESCs (Fig. 1d, Supplementary Table 4). Moreover, we did not find a significant enrichment of a 4-cell stage signature in EPSCs compared with ESCs (Extended Data 2d, Supplementary Table 5). We also observed that D-EPSCs could 6 be readily converted back to 2 iLif.

8 Single-cell RNA-seq (scRNA-seq) is particularly suited to resolve cellular heterogeneity and 9 identify subpopulations with distinct transcriptional features. To examine whether totipotent 10 features can be detected in individual cells, we applied SMART-seq2 scRNA-seq to ESCs and 11 EPSCs derived from them. As a reference, we transcriptionally tracked mouse pre- and post12 implantation development from zygote to E6.75, and included naïve ESCs and primed EpiSCs as

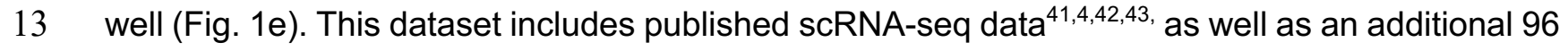
14 cells from E2.5 and E4.5 embryos, and 551 cells from three PSC conditions: ESCs (2iLif), L15 EPSCs and D-EPSCs, sequenced in this study. This integrated dataset provided suitable sampling for establishing a comparison of embryonic development with the different stem cell

17 culture conditions (Extended Data 2e and Methods). Based on clustering, we resolved clear 18 segregation of ICM/TE and EPI/PE lineages and annotated them based on the expression of well19 established lineage markers (Extended Data 2f). As expected, ESCs cultured in 2iLif occupied 20 the space between E3.5 ICM and E4.5 EPI, while primed EpiSCs clustered with E5.5 and E6.75 21 EPI cells. We found that along the developmental trajectory, L-EPSCs and ESCs clustered 22 between the E3.5 ICM and E4.5 EPI stages, while the majority of D-EPSCs clustered together 23 with E5.5 stage EPI cells (Fig. 1e). Although the majority of D-EPSCs clustered with E5.5 EPI, 24 they also expressed several naïve-associated transcripts suggesting a combined expression of 25 naïve and more primed signatures (Extended Data Fig. 3a). Using scRNA-seq we detected 26 enrichment of 4-cell stage genes in EPSCs over 2iLif for both L-EPSCs and D-EPSCs, 
1 corroborating previous findings (Extended Data $2 \mathrm{~g})^{35}$. The differences in detection of the 4-cell

2 signature between bulk and scRNA-seq may be attributed to technical differences between the

3 methods.

4 We also observed that top differentially expressed genes and gene modules A and B previously

5 reported to be upregulated in D-EPSCs compared to Lif/serum-cultured ESCs were also

6 upregulated in D-EPSCs compared to 2iLif-cultured ESCs (Extended Data 2h,i). We also

7 compared each stem cell condition independently with each developmental stage (Fig. 1f). While

8 ESCs showed significant correlation with all preimplantation stages and highest similarity with

9 E4.5 EPI ( $r=.72, p<.001)$, L-EPSCs (from all studies) exhibited the most resemblance to E4.5 EPI

$10 \quad(r=.64, p<.001)$, while lacking significant correlation with other developmental stages $(p>.05)$.

11 Consistent with the UMAP, D-EPSCs correlated the most with E5.5 EPI $(r=.89, p<.001)$, but also

12 showed close similarity with primed EpiSCs $(r=.71, p<.001)$, and E6.75 EPI $(r=.51, p<.001)$. The

13 position occupied by L-EPSCs in the UMAP space is consistent with the original report ${ }^{35}$ in the

14 sense that the cells do not cluster closer with the totipotent cells of the morula. We could detect

15 a small fraction of D-EPSC and 2iLif cultured cells positioned with the embryonic TE cluster (3

16 and 6 cells respectively) and PE cluster ( 5 and 14 cells respectively) (Fig. 1 e and Extended Data

17 3a). Importantly, this appeared to be an effect of the integration, scattering the transcriptional

18 profiles rather than representing true heterogeneity as these cells did not show distinct profiles

19 prior to integration (Extended Data 3a). In conclusion, L- and D-EPSCs single-cell transcriptomes

20 are distinct from each other and align with pluripotent rather than totipotent states.

22 Embryo development is under the control of transcription factors that bind to cis-regulatory

23 regions, forming gene regulatory networks. We reconstructed gene regulatory networks active in

24 early development, ESCs and EPSCs, from scRNA-seq data, using single-cell gene regulatory

25 inference and clustering (pySCENIC ${ }^{44,45}$ ). SCENIC predicts TFs that may control cellular states

26 present in the dataset, together with candidate TF target genes. A TF and its candidate targets 
1 are called a regulon, and by quantifying the activity of regulons in each single cell, SCENIC can

2 be used to cluster cells based on the activity of regulatory programs. In contrast to identifying the

3 global transcriptional state of cells, here we project a UMAP visualization based on regulon activity

4 and reveal that 2iLif ESCs localize closest to E3.5 ICM, and both L- and D-EPSCs clustered

5 between E4.5 EPI and E5.5 EPI (Extended Data 3b). These results show that the regulatory state

6 of EPSCs resembles that of late pluripotent EPI rather than earlier, totipotent developmental

7 stages.

9 Capacity of EPSCs to enter the trophectoderm program and generate TSC-like cells in vitro

11 Another test to judge totipotency is to evaluate the capacity of cells to enter the trophoblast

12 lineage. This can be assessed in vitro by switching cells to TSC culture conditions and assaying

13 whether cells give rise to TSC-like cells, a transition that pluripotent ESCs cannot make. When

14 bulk L-EPSCs cultures were switched to TSC conditions followed by RT-PCR to assay trophoblast

15 marker gene expression (Fig. 2a), no substantial activation of such genes was seen (Fig. 2b).

17 To examine whether a small subpopulation of EPSCs may harbor the potential to directly convert

18 into TSCs, as suggested by a previous study $\left(\mathrm{Yang}^{32}\right)$, we analyzed the expression of key TSC

19 markers on a single cell level using flow cytometry (Fig. 2c, Extended Data 4). Additionally, we

20 tested whether EPSCs can be reprogrammed into TSC-like cells more efficiently than ESCs.

21 While ESCs do not readily convert into TSCs, they can be reprogrammed with low efficiency into

22 TSCs by induced overexpression of TSC-associated TFs ${ }^{22}$. To assay TSC reprogramming, we

23 used a tetracycline-inducible $\mathrm{Cd} \times 2(\mathrm{iCdx} 2)$ and Oct4 heterozygous ESC background, used in the

24 original ESC-to-TSC reprogramming experiments ${ }^{22}$. We also established an Elf5-2A-mCherry

25 reporter ESC line by targeting $2 \mathrm{~A}$-mCherry to the C-terminus of the endogenous Elf5 locus s, $^{46,47,23,25}$

26 (Fig. 2c). We then switched EPSCs and ESCs to TSC medium (with Fgf4) with or without 
1 tetracycline $(+/-4 \mathrm{OH})$ and cultured the cells for 6 days $(6 \mathrm{~d})$ before analyzing TSC-marker

2 expression by immunostaining for CD40 and Plet1 followed by flow cytometry. We found that

3 Cdx2 induction did not increase reprogramming efficiency of EPSCs compred with ESCs (Fig.

4 2d). In contrast, a control TSC line in which we also targeted the Elf5 gene with the mCherry

5 reporter showed 80\% CD40/PLET1/ELF5 triple-positive cells (Fig. 2e). Collectively, these results

6 indicate that the EPSC states do not facilitate more efficient reprogramming into TSCs in vitro, in

7 contrast with a previous study that suggested an increased ability of EPSC to give rise to TSC-

8 like cells compared to $\mathrm{ESCs}^{36,35}$.

10 In vitro blastoid-forming ability of D-EPSCs based on Li et al. 2019 and Sozen et al. 2019

12 The ultimate proof of totipotency is the ability of a single cell type, or more stringently a single cell,

13 to give rise to an entire blastocyst and, subsequently, a viable and fertile animal. Recently,

14 blastocyst-like structures, termed blastoids, have been generated in vitro from different stem cell

15 types ${ }^{48,49,39,40}$. These protocols use different combinations of growth factors and inhibitors to

16 generate blastoids, which in multiple aspects resemble real blastocyst stage embryos, although

17 until now, none have been able to generate viable animals. Most notably, two recent reports used

18 D-EPSCs, either as a sole stem cell source (Belmonte group, B-blastoid) ${ }^{39}$ or in combination with

19 TSCs (Zernicka-Goetz group, ZG-blastoid) ${ }^{40}$ to generate blastoids. Importantly, a large proportion

20 of the blastoid cells generated with only D-EPSCs showed expression of genes previously

21 associated with post-implantation stage lineages and not cells of the blastocyst. We, therefore,

22 re-analyzed the scRNA-seq data provided in these reports and aligned them to our existing

23 sampled pre-implantation cells, along with an additional dataset containing cells up to E7.50 (Fig.

24 3a, Extended Data 5a). Resource data are also available for visualization in SCope ${ }^{51}$. ZG-

25 blastoids were generated by combining either D-EPSCs or ESCs with TSCs, using a modified

26 version of the protocol established by Rivron et al. for making blastoids ${ }^{48}$ with ESCs and TSCs. 
1 Corroborating previous findings, we found that both ESCs and D-EPSCs are able to give rise to

2 similar cell types in these blastoids (Extended Data $5 b, c)$ : to cells resembling the E4.5 blastocyst

3 EPI and to cells most similar to E4.5 PE or post-implantation parietal endoderm (Fig. 3a, Extended

4 Data 5c). However, using the ZG-blastoid forming method, the authors saw no detectable

5 contribution of D-EPSCs towards the TE lineage and blastoid formation was not observed when

6 TSCs were omitted ${ }^{40}$. B-blastoids, made from only D-EPSCs, also contained cells (B-blastoid EPI)

7 that grouped with E4.5 blastocyst EPI cells and cells (B-blastoid PE) that grouped towards E4.5

8 blastocyst PE or post-implantation parietal endoderm cells (Fig. 3a, Extended Data 5d). However,

9 only $6.7 \%$ of B-blastoid cells (B-blastoid-TE) clustered close to the TE lineage, between blastocyst

10 TE cells and post-implantation ExE (Extended Data $5 \mathrm{e}$ ). The remaining $60 \%$ of B-blastoid cells

11 consisted of two intermediate clusters (B-blastoid-intermediate-1 and 2) that did not align with any

12 blastocyst cells but instead resembled most closely certain post-implantation stage embryo cells.

13 In an in vitro blastoid culture, these cells could be mistaken for TE cells as they express Cdx2,

14 Krt8 and Krt18. However, they also co-expresse T (Brachyury), suggesting an embryonic or extra-

15 embryonic mesoderm identity (Fig. 3b). Indeed, B-blastoid-intermediate-1 cells showed

16 significant correlation with postimplantation E5.5-7.5 EPI and ExE mesoderm $(r=.33, p<.001)$ and

17 B-blastoid-intermediate-2 cells with ExE mesoderm ( $r=.67, p=.001)$ and other mesodermal

18 lineages: mixed mesoderm ( $r=.64, p=.001)$, intermediate mesoderm ( $r=.62, p=.001)$, nascent

19 mesoderm ( $r=.48, p=.001)$, as well as a strong resemblance to mesenchyme $(r=.42, p=.001)$ (Fig.

20 3c). Nevertheless, the small subpopulation of B-blastoid-TE cells resembling blastocyst TE is

21 intriguing, and leaves the door open to the possibility that D-EPSCs can contribute to the

22 trophoblast lineage under B-blastoid forming conditions. However, it should be noted that only a

23 fraction of these cells expressed key TE TFs such as Cdx2, Elf5 and Gata3 and at levels that

24 were very low compared to embryo TE cells. These cells also exhibited a higher similarity to ExE

25 ectoderm ( $r=.74, p=.001)$, than genuine TE (r=.62, $p=.001)$ (Fig. 3b.c). 
1 At the gene regulatory level, both B- and ZG-blastoid cells aligned well with embryo cells,

2 indicating that the gene regulatory programs of developing blastocysts are recapitulated to a large

3 extent in blastoids derived from D-EPSCs (Extended Data 6a, Supplementary Figure 1). For

4 example, the activity of selected regulons for lineage-specific TFs showed that blastoid-generated

5 PE and EPI cells shared regulatory activity with their respective cell types in E4.5 blastocysts

6 (Extended Data 6b). Furthermore, in-depth analysis of target genes of NANOG and GATA4, EPI

7 and PE TFs, respectively, showed similar target gene expression patterns between blastoids and

8 embryos (Extended Data 6c). However, the TE regulatory state did not seem to be well

9 recapitulated in D-EPSC-derived blastoids. Regulons associated with TE, such as GATA3, CDX2,

10 PITX1 and SOX6 are less expressed in blastoid TE compared to embryo TE (Extended Data 6b).

11 Indeed, GATA3 target genes are less expressed in blastoid TE cells compared with embryos

12 (Extended Data 6c), indicating that the misregulation of specific parts of the regulatory program

13 underlying embryogenesis may limit blastoid development. We also investigated the regulatory

14 activity of intermediate blastoid subpopulations to determine what may prevent these cells from

15 becoming appropriate lineages present in the blastocyst. In line with gene expression analysis,

16 we found that the intermediate blastoid populations (B-blastoid-intermediate-1 and 2) activated

17 regulons of post-implantation EPI and mesodermal lineages (Extended Data 6a,b) such as T and

$18 \mathrm{MIXL} 1$. We, therefore, analyzed gene expression of $\mathrm{T}$ target genes and found that B-blastoid-

19 Intermediate-2 cells activate most, but not all, $T$ targets (Extended Data 6c). At the same time,

20 however, these cells, as well as mesodermal cells, also activate many targets of CDX2 (Extended

21 Data 6c). This suggests that B-blastoid-intermediate-2 cells may have failed to activate the TE

22 regulatory program and instead arrested between overlapping mesodermal and TE states.

23 Altogether, these results demonstrate that the gene regulatory programs active in embryos are,

24 to a large extent, engaged in EPSC-derived blastoids, but not fully, which might contribute to the

25 developmental arrest of these structures. 
In vivo lineage contributions of totipotent blastomeres and EPSCs at E4.5

3 The capacity to enter trophoblast lineage can also be assessed in vivo by creating chimeras with

4 a host embryo and analyzing lineage contributions at later developmental time points. To test

5 lineage contributions of truly totipotent cells, we aggregated a morula-stage (E2.5) embryo (host)

6 with a single blastomere from an 8-cell stage embryo (donor), as most, if not all blastomeres at

7 the 8-cell stage are considered totipotent ${ }^{52,53}$. We allowed chimeras to develop for 48 hours before

8 analyzing lineage contributions at the late blastocyst stage (E4.5). At E4.5 the three blastocyst

9 lineages are clearly segregated (Fig. 4a) and express well-characterized lineage-specific

10 markers, such as Sox2 (EPI), Sox17 (PE) and Cdx2, Gata3, Krt8, and Krt18 (TE) ${ }^{54,55,56,57,17,58}$. To

11 visualize progeny of the donor cell, we isolated single blastomeres from embryos expressing

12 either H2B-Gfp (nuclear-localized marker) or DsRed (no nuclear localization, marker appears in

13 both cytoplasm and nucleus) and used wild-type embryos as hosts. We found that in $60-70 \%$ of

14 chimeras the donor blastomere contributed to both the inner cell mass (EPI + PE) and the TE

15 (Fig. 4b), which was verified by co-immunostaining with lineage-specific markers (Fig. 4c). These

16 data serve as a benchmark for lineage contributions of truly totipotent cells in a chimera.

18 We then aggregated EPSCs or control parental ESCs to wild-type host embryos and analyzed

19 chimeras at E4.5. In contrast to blastomeres which often contributed to both TE and ICM cells,

20 the great majority of stem cell progeny contributed exclusively to the EPI (Fig. 4d). Interestingly,

21 we observed that progeny of both L-EPSCs and D-EPSCs localized to trophectodermal positions

22 in 13 and $14 \%$ of chimeras, respectively, while progeny of the parental ESC line cultured in 2iLif

23 conditions localized mainly to the epiblast, corroborating previous studies ${ }^{36,35}$ (Fig. 4d). However,

24 when we immuno-stained chimeras for epiblast (Sox2) and trophectoderm markers (Cdx2), none

25 of the L-EPSC or D-EPSC derived cells, or the occasional 2iLif ESCs in the TE position showed

26 expression of either marker (Fig. 4e and Extended Data 7). Therefore, we conclude that EPSCs 
1 can contribute cells that localize to the TE but do not express a key TE marker. We noted that

2 even D-EPSCs had high chimeric potential in the epiblast, likely due to these cells not only 3 expressing primed, but also naïve markers (Extended Data 3).

$5 \quad$ In vivo lineage contributions of totipotent blastomeres and EPSCs at E6.25

7 To confirm that the observed lineage contributions at E4.5 persist later in development, we 8 examined chimeras post-implantation, at E6.25. Shortly after implantation, the EPI forms a cup9 shaped epithelium, the PE forms the two layers of the visceral and parietal endoderm, and the TE 10 cells overlying the EPI proliferate to form the extra-embryonic ectoderm (ExE) and the 11 ectoplacental cone (Fig. 5a). First, we generated chimeras with H2B-Gfp expressing blastomeres

12 and found that progeny of the blastomeres can contribute to both the Oct4-positive EPI, the 13 Tfap2c and Elf5-positive ExE and the Tfap2c-positive ectoplacental cone (Fig. 5b). Next, we 14 generated chimeras with H2B-Gfp expressing EPSCs or ESCs and performed similar lineage 15 analysis at E6.25. We found that all cell types readily contributed to the EPI lineage of the host 16 embryo (Fig. 5c). Interestingly, while ESC and D-EPSC chimeras occasionally contained donor 17 cells in the trophoblast compartment ( $5 \%$ of chimeras), we also found that around $25 \%$ of L18 EPSC chimeras contained a few cells in the ExE. However, when we performed immuno-staining 19 for lineage-specific markers, cells localized to trophoblast regions did not express trophoblast 20 markers such as Elf5 and Tfap2c but did express EPI marker Oct4 (Fig. 5d and Extended Data 21 7). These data emphasize that donor cell localization alone does not necessarily indicate 22 appropriate lineage-specific marker allocation. We also show an increased frequency of 23 mislocalized L-EPSCs in chimeras, which may potentially explain the previously reported behavior 24 of these cells. 
In vivo lineage contributions of totipotent blastomeres, L-EPSCs and ESCs in E12.5 placentas

4 To test whether ExE-localized donor cells in chimeras give rise to differentiated trophoblast cell

5 types, we analyzed chimeric placentas at E12.5. The placenta has a complex structure and

6 contains both trophoblast as well as embryo-derived cell types ${ }^{59}$ (Fig. 6a). Additionally, due to its

7 high metabolite content, the placenta exhibits elevated levels of autofluorescence ${ }^{60-63}$. These

8 properties make immuno-fluorescent lineage analysis in the placenta a tricky task, requiring

9 thorough evaluation aided by appropriate positive and negative controls. First, we identified

10 antibodies and immuno-fluorescent staining conditions to label the different cell types of the

11 placenta. We used Mct1 and Mct4 to label syncytiotrophoblast I and II, respectively, Tpbpa to

12 label spongiotrophoblast, and Krt8, Cdh3 and Tfap2c to label all trophoblast cell types in both the

13 spongio and the labyrinth zones. Tfap2c is a nuclear-localized TF, making it an ideal marker to

14 detect co-localization with nuclear-localized lineage tracers (e.g., H2B-Gfp). Finally, we used

15 CD31 to label embryo-derived endothelial cells in the placenta. We then used this marker panel

16 to show that in chimeric placentas generated with a single H2B-Gfp or DsRed expressing

17 totipotent blastomere and a wild-type host embryo, blastomere progeny contribute to all analyzed

18 lineages (Extended Data 8). To unambiguously distinguish between trophoblast and embryo-

19 derived cells in the placenta, we took advantage of a technique termed tetraploid

20 complementation, which involves generating a chimera using a tetraploid host embryo and diploid

$21 \mathrm{ESCs}^{64,65}$ (Fig. 6b). Tetraploid cells are not tolerated in the embryonic compartment and ESCs do

22 not contribute to the trophoblast compartment. Therefore, any surviving conceptus at E12.5

23 consists of trophoblast originating from tetraploid cells and embryonic tissues originating from

24 ESCs. We generated chimeras in which either the tetraploid cells or ESCs (Fig. 6c) carried H2B-

25 eGfp and immuno-stained placental sections at E12.5 for the markers described above (Extended

26 Data 9). As expected, we saw that in chimeras with H2B-eGfp-posititve tetraploid cells, 
1 trophoblast markers (Tfap2c, Cdh3, Tpbpa, and Mct4) always overlapped with the Gfp signal,

2 while the embryonic marker CD31 did not. In contrast, in chimeras with H2B-eGfp-posititve ESCs

3 only CD31 overlapped with the Gfp signal, and trophoblast markers were excluded from Gfp-

4 positive cells. This panel highlights the difficulty in distinguishing different cell types in the

5 placenta, especially in the labyrinth zone, without a detailed analysis of markers and also

6 emphasizes the challenge of matching a nuclear label with a membrane-localized signal in

7 individual cells. Co-localization can be interpreted more clearly when the fluorescent lineage

8 tracer and the cell-type specific marker are in the same subcellular compartment, as exemplified

9 in our staining panel by the co-localization of H2B-eGfp and Tfap2c. Next, we generated chimeric

10 placentas using diploid host embryos and L-EPSCs and analyzed them using the same marker

11 panel (Fig. 6d and Extended Data 8). We could only detect Gfp-positive cells in the embryonic,

12 but not in the trophoblast compartment, suggesting that L-EPSCs do not readily give rise to

13 differentiated trophoblast cell types.

\section{Discussion}

18 Here we present different criteria to evaluate the differentiation potential of early ESCs. We 19 provide a large compiled dataset of single cell transcriptomes covering different in vivo cell types 20 from fertilization to gastrulation, as well as several PSC types, which can be used to map 21 previously unknown cell types based on transcriptional similarities. As a resource for the 22 community, the data presented here are made available through a user-friendly .loom file that can 23 be explored online (https://github.com/pasquelab/totipotency).

24 We demonstrate assays to test the differentiation potential of cells by conversion or 25 reprogramming to the TE fate and by chimera and lineage contribution analyses in vivo. We 26 focused on the TE, hence cannot draw conclusions about PE contribution. We highlight the 
1 importance of evaluating chimeric contributions not only based on the localization of donor cells,

2 but also on the expression on lineage-specific marker analysis.

3 Using these criteria, we examine the potential of two reported EPSCs. Surprisingly, we fail to find

4 convincing evidence that these cell types harbor extensive expanded or extended potential.

5 Instead, based on transcriptomic comparisons L-EPSCs most closely resemble E4.5 EPI cells or

6 the parental ESCs cultured in 2iLif and D-EPSCs the E5.5 EPI or EpiSCs. In TSC conversion or

7 reprogramming settings, neither L-EPSCs or D-EPSCs show enhanced potential compared to

8 parental ESCs. Finally, in chimeric experiments, EPSCs only show a convincing contribution to

9 the EPI lineage. Interestingly, we found that in chimeras analyzed at the late blastocyst stage

10 generated with both L-EPSCs or D-EPSCs, but not ESCs, cells occasionally localized to TE

11 positions but did not express either EPI nor TE markers. Occasional mislocalized L-EPSCs in

12 post-implantation chimeras also lacked trophoblast marker expression. These results suggest

13 that cell adhesion differences might contribute mislocalized cells, but this remains speculative.

15 Our results suggesting that EPSCs are unable to enter the trophectoderm lineage are seemingly

16 in contrast with a recent study reporting the formation of blastocyst-like structures, termed

17 blastoids, using only EPSCs ${ }^{39}$. Re-examining blastoid scRNA-seq data corroborates the idea that

18 EPSCs are able to engage the gene regulatory programs utilized in distinct cellular lineages of

19 blastocysts of natural embryos. However, we also found differences between the gene regulatory

20 programs of blastocyst embryos and EPSC-derived blastoids, which were most apparent in the

21 TE lineage. Only $6.7 \%$ of cells from D-EPSCs-derived blastoids were categorized as TE and

22 even these showed an ExE-like profile. These TE-like cells also lacked robust expression of

23 several classical TE TFs. Problematically, the most abundant cell types in B-blastoids (B-blastoid

24 intermediate 1 and 2) seem to most closely resemble mesoderm, expressing markers such as $T$,

25 yet also share several common markers with the trophoblast lineage. With such cell composition,

26 it is not surprising that B-blastoids are not able to generate a live conceptus. Although not 
1 abundant, the presence of TE or ExE-like cells in B-blastoids is intriguing and leaves the door

2 open for the possibility that some EPSCs may indeed harbor the potential to differentiate into

3 trophoblast. Of note, EpiSCs undergoing reprogramming to the naïve ESC state have also been

4 reported to form blastoids and seem to at least partially recapitulate the gene regulatory programs

5 of blastocysts ${ }^{66}$. Moreover, perturbations of Sox 2 expression in naïve PSCs may lead to increased

6 trophoblast differentiation potential ${ }^{67}$.

7 Why is TE potential only revealed in the blastoid-forming assays and not in the context of

8 chimeras? Differences in the behaviour of the cells in embryo aggregation and blastoid assays

9 may explain why TE potential is only revealed in blastoid assays and not in the context of a

10 chimera. Forcing cells to the surface of a forming sphere in the blastoid method may mimic TE-

11 inducing cues better than aggregation assays. In aggregation assays donor cells have positional

12 freedom within the host embryo, which permits donor cells to group with the developing TE or

13 ICM compartments ${ }^{4}$. Therefore if only the potential exists for TE fate, this may not be realized in

14 an aggregation setting. Additionally, B-blastoids are formed under specific culture conditions,

15 which may direct differentiation more robustly than the environment of the embryo. Supporting

16 this notion, D-EPSCs were not able to give rise to a TE-like layer when combined with TSC under

17 a different blastoid forming protocol (ZG-blastoid) ${ }^{40}$. It should, however, still be considered that

18 the TE or ExE-like blastoid cells fail to express Cdx2, Gata3 and Elf5 transcripts at similar levels

19 to endogenous TE or ExE of the embryo suggesting that their transcriptional profile still is distinct

20 from in vivo cells.

21 Notably, the B-blastoid method employs Bmp4 and inhibits Activin/Nodal signaling ${ }^{39}$, conditions

22 which were also used in another blastoid protocol by the Tomoda lab ${ }^{49}$. The Tomoda group used

23 EpiSCs as starting cells and were also able to produce blastoids with certain TE-like marker

24 expression in the surface layer. Additionally, high Bmp4 and low Activin/Nodal was previously

25 computationally predicted and shown in vitro to activate TE-marker gene expression in ESCs in 
1 which Jak/Stat signaling was inhibited ${ }^{68}$. These data suggest that high Bmp4 and low

2 Activin/Nodal signaling may be key to TE-like cell induction.

3 Importantly, these signaling conditions are also involved in inducing proximal mesoderm fates

4 during gastrulation and Bmp induces mixed mesoderm and trophoblast differentiation in EpiSCs

5 and $\mathrm{hESCs}{ }^{69}$, consistent with the appearance of abundant mesoderm-like cells in B-blastoids.

6 Could the starting stem cell state be crucial for facilitating mesoderm versus trophoblast

7 differentiation? Indeed, it was shown that Cdx2 overexpression in ESCs induces reprogramming

8 into TSCs, while Cdx2 overexpression in EpiSCs results in mesodermal gene expression ${ }^{22,70}$,

9 highlighting the importance of the starting state for different differentiation outcomes. Notably, as

10 our analysis placed D-EPSCs closest to primed EpiSCs, the widespread induction of mesodermal

11 profiles is not surprising.

12 Altogether, we propose that to truly unlock a cell's differentiation potential into all extra-embryonic

13 and embryonic lineages, a starting state more resembling earlier cleavage stages of development,

14 is needed. Our study highlights this challenge and sets gold standards for evaluating the

15 differentiation potential of any type of early stem cell including $2 \mathrm{C}$-like cells in the future.

\section{Acknowledgments}

18 The authors wish to acknowledge the contribution of the Model Production Core staff at the Center

19 for Phenogenomics for technical support. Single-cell sequencing was performed at National

20 Genomics Infrastructure in Stockholm at Science for Life Laboratory (funded by the Knut and

21 Alice Wallenberg Foundation and the Swedish Research Council) with assistance from

22 SNIC/Uppsala Multidisciplinary Center for Advanced Computational Science with massively

23 parallel sequencing and access to the UPPMAX computational infrastructure. Bulk RNA

24 sequencing was performed in KU Leuven Genomics Core. We would like to thank Stein Aerts,

25 Sara Aibar, Chris Davie and Christopher Flerin for discussions and support. We thank Dr. Jun Wu

26 for the C1-12 tdTomato-expressing D-EPSCs. This work was funded by CIHR (FDN-143334), 
1 Genome Canada and Ontario Genomics (OGI-099), Programme de bourses de chercheur-

2 boursier FRQS Junior 1 (FRQS 268829, 280187), the Swedish Research Council, Ragnar

3 Söderberg Foundation, Ming Wai Lau Center for Reparative Medicine, Center for Innovative

4 Medicine, Wallenberg Academy Fellow, NSERC (2014-04497). Research in the Pasque lab is

5 supported by The Research Foundation-Flanders (FWO) (Odysseus Return Grant G0F7716N to

6 VP, FWO grants G0C9320N and G0B4420N to VP), the KU Leuven Research Fund (BOFZAP

7 starting grant StG/15/021BF to VP, C1 grant C14/16/077 to VP and project financing), and FWO

8 Ph.D. fellowships to A.Ja (1158318N), IT (1S72719N) and SKT (1S75720N).

10 Author contributions

11 J.P.S., A.M., A.Ja., T.P., M.E.B., N.D.G., S.K.T. performed in vitro stem cell experiments. E.P.,

12 I.R., B.B. performed in vivo chimera experiments. J.P.S., A.Ja., P.K., S.P., M.E.B., I.T., F.L.,

13 V.P., performed transcriptional analyses. E.P., V.P., F.L., J.P.S., A.Ju. and J.R., planned

14 experiments, analyzed data and wrote the manuscript. V.P., F.L., E.P., and J.R., conceived the 15 study.

\section{Additional Information}

18 Supplementary material is available for this paper.

19 Correspondence and requests for materials should be addressed to Janet Rossant

20 (janet.rossant@sickkids.ca), Eszter Posfai (eposfai@princeton.edu), Fredrik Lanner

21 (fredrik.lanner@ki.se), Vincent Pasque (vincent.pasque@kuleuven.be).

22 Reprints and permissions information is available at www.nature.com/reprints.

\section{Competing Financial Interest}

25 Authors declare no competing financial interests. 
2 Figures

3 Fig. 1: Gene expression analysis of candidate totipotent stem cells using RNA-seq

4 a) Experimental design of L-EPSC conversion. Mouse ESCs grown in 2iLif were switched to L-

5 EPSC medium and subjected to bulk RNA-seq at day (D) 0, D3, D5, D7, D9, D11, D13 and

6 D15.

7 b) The L-EPSC transcriptome was rapidly induced during conversion from 2iLif ESCs. Principal

8 Component Analysis (PCA) analysis of bulk RNA-seq data of samples from different timepoints

9 of L-EPSC conversion and 2iLif ESCs.

10 c) PCA analysis of bulk L-EPSC transcriptomes after integrating the dataset from this study with

11 published bulk RNA-seq data (Yang et al. 2017 ${ }^{35}$ ) and scRNA-seq data (Deng et al. 2014 ${ }^{41}$;

12 Posfai et al. 20174; Yang et al. 2017 35 ).

13 d) 4-cell stage embryo marker genes are expressed in a similar pattern in EPSCs and ESCs.

14 Expression of 4-cell signature genes in ESCs undergoing conversion to L-EPSCs, published

15 bulk RNA-seq data (Yang et al. 2017 ${ }^{35}$, Yang et al. $2017^{36}$ ) and scRNA-seq data (Deng et al.

$162014^{41}$; Yang et al. $2017^{35}$ ).

17 e) Single-cell UMAP comparing developmental progression from zygote to E6.75 EPI, including 18 pre-implantation extra-embryonic lineages, with L-EPSCs, previously published L-EPSCs ${ }^{35}$, D-

19 EPSCs, 2iLif ESCs and primed EpiSCs.

20 f) Correlation matrix based on top 2000 expressed genes averaged tracking zygote to E6.75

21 EPI, including pre-implantation extra-embryonic lineages, with L-EPSCs, D-EPSCs, 2iLif ESCs

22 and primed EpiSCs.

24 Fig. 2: In vitro capacity and potential of candidate totipotent stem cells to give rise to 25 trophoblast stem cells 
1 a) Differentiation of L-EPSCs to TSCs. 2iLif ESCs or L-EPSCs were subjected to TSC

2 differentiation conditions. RNA was collected at D0, D3, D6, D9, D12 and qPCR was used to

3 measure the expression of TE marker genes.

4 b) Silencing of extra-embryonic gene expression was maintained in L-EPSCs exposed to TSC

5 differentiation conditions. Expression of TSC markers: Elf5, Eomes, Tfap2c, Cdx2, Asc/2, Esx1

6 and Nanog as control at D3, D6, D9 and D12 of differentiation by RT-qPCR. $n=2$ independent

7 experiments.

8 c) Experimental design of ESC, L-EPSC or D-EPSC to TSC conversion $(-4 \mathrm{OH})$ or

9 reprogramming experiments. ESC, L-EPSC or D-EPSC lines harbored a tamoxifen(4OH)-

10 inducible Cdx2 transgene, were heterozygous for Oct4 (Pou5f1) and contained an Elf5-2a-

11 mCherry reporter. Cells switched to TSC media with or without $4 \mathrm{OH}$ were analyzed by flow

12 cytometry on indicated days for TSC markers CD40, ELF5 and PLET1.

13 d) Flow cytometry analysis of ESC, L-EPSC or D-EPSC cells switched to TSC media with or

14 without $4 \mathrm{OH}$ on indicated days. Percentages of cells positive of each TSC marker, or cells

15 positive for all three markers are shown. $n=2$ (D-EPSCs) or $n=3$ (2iLif and L-EPSCs) biological

16 replicates were performed, error bars indicate standard deviation of the mean. For each marker

17 (singles or triple) pairwise two-sided t-tests were performed between cell types at each

18 timepoint and $4 \mathrm{OH}$ condition - none were found to be statistically significant.

19 e) Flow cytometry analysis of TSCs containing an Elf5-2s-mCherry reporter. Percentages of

20 cells positive of each TSC marker, or cells positive for all three markers are shown. $n=3$

21 biological replicates were performed, error bars indicate standard deviation of the mean.

23 Fig. 3: Single cell transcriptional comparison of blastoid cells to in vivo and different

\section{4 stem cell states}

25 a) Single-cell UMAP tracking morula to E7.5 gastrulation in embryonic and extra-embryonic

26 lineages, comparing all stem cells, B-blastoid cells and ZG-blastoid cells. 
1 b) Dot plot representing the frequency of expression and average expression for select lineage

2 marker genes.

3 c) Matrices showing correlation coefficients between B-blastoids (top) or ZG-blastoids (bottom)

4 and selected lineages.

6 Fig. 4: In vivo potential of candidate totipotent stem cells to give rise to the

7 trophectoderm lineage at E4.5

8 a) Cartoon showing cell types of the E4.5 blastocyst embryo.

9 b) Stacked bar chart showing percent of E4.5 chimeric embryos with different lineage

10 contributions. Chimeras were generated by aggregating a wild-type host embryo and a single

11 blastomere of an 8-cell stage embryo that expressed either H2B-Gfp (number of chimeras

12 analyzed: 45) or DsRed (number of chimeras analyzed: 43). The number of chimeric embryos

13 showing different lineage contributions (inner cell mass - ICM, trophectoderm -TE, inner cell

14 mass and trophectoderm - ICM + TE) is indicated within the bar chart. c) Immunofluorescent

15 stainings of E4.5 chimeric embryos with lineage contributions to both ICM and TE generated

16 with a single blastomere of an 8-cell stage embryo that expressed either H2B-Gfp (top panel) or

17 DsRed (lower panel). Stained for Sox2+Sox17 (ICM), Nanog (EPI), Cdx2, Gata3, Krt8 and Krt18

18 (TE) and Gfp or DsRed. Left panel shows maximum intensity projection of whole chimera, scale

19 bar: $40 \mu \mathrm{m}$. Right panels show magnified single plane images of ICM and TE (bottom)

20 contributions, scale bar: $10 \mu \mathrm{m}$. For each antibody combination, at least 6 chimeric embryos

21 were analyzed, yielding similar results.

22 d) Stacked bar chart showing percent of E4.5 chimeric embryos with different lineage

23 contributions. Chimeras were generated by aggregating a wild-type host embryo with H2B-Gfp

24 expressing ESCs (number of chimeras analyzed: 32), L-EPSCs (number of chimeras analyzed:

2534 ) or D-EPSCs (number of chimeras analyzed: 40). The number of chimeric embryos showing

26 different lineage contributions (epiblast - EPI; trophectoderm -TE; epiblast and trophectoderm 
1 position - EPI + TE position) is indicated within the bar chart. e) Example of E4.5 chimeric

2 embryos generated with H2B-Gfp expressing D-EPSCs stained for Sox2 (EPI), Cdx2 (TE) and

3 Gfp. Left panel shows maximum intensity projection of whole chimera, scale bar: $40 \mu \mathrm{m}$. Right

4 panel shows magnified single plane image of Gfp positive D-EPSC contributions, scale bar: 10

$5 \mu \mathrm{m} .40$ chimeric embryos were analyzed, of which 5 showed cells in TE positions (numbers in

6 panel d).

8 Fig. 5: In vivo potential of candidate totipotent stem cells to give rise to the trophoblast

9 lineage at $\mathrm{E} 6.25$

10 a) Cartoon showing cell types of the E6.25 embryo.

11 b) Immunofluorescent stainings of E6.25 chimeric embryos with lineage contributions to both

$12 \mathrm{EPI}$ and trophoblast lineages generated with a single blastomere of an 8-cell stage embryo that

13 expressed H2B-Gfp. Stained for Oct4 (EPI), Tfap2c and Elf5 (trophoblast compartment) and

14 Gfp. Left panel shows maximum intensity projection of whole chimera, and right panels show

15 single plane images, scale bar: $100 \mu \mathrm{m} .18$ embryos were analyzed.

16 c) Stacked bar chart showing percent of E6.25 chimeric embryos with different lineage

17 contributions. Chimeras were generated by aggregating a wild-type host embryo with H2B-Gfp

18 expressing ESCs (number of chimeras analyzed: 14), L-EPSCs (number of chimeras analyzed:

19 26) or D-EPSCs (number of chimeras analyzed: 40). The nuber of chimeric embryos showing

20 different lineage contributions (epiblast - EPI, trophoblast (extraembryonic ectoderm and

21 ectoplacental cone) - TB, epiblast and trophoblast position - EPI + TB position) is indicated

22 within the bar chart. d) Examples of E6.25 chimeric embryos generated with H2B-Gfp

23 expressing L-EPSCs stained for Oct4 (EPI), Tfap2c and Elf5 (trophoblast compartment) and

24 Gfp. Left panel shows maximum intensity projection of whole chimera, scale bar: $100 \mu \mathrm{m}$. Right

25 panel shows magnified single plane image of Gfp positive L-EPSC contributions, scale bar: 50 
$1 \mu \mathrm{m} .26$ cimeric embryos were analyzed, of which 7 showed cells in trophoblast positions (4 were

2 immunostained for Tfap2c and Elf5 and 3 were immunostained forTfap2c and Oct4).

5 Fig. 6: In vivo potential of candidate totipotent stem cells to give rise to the trophoblast

$6 \quad$ lineage of the E12.5 placenta

7 a) Cartoon indicating cell types and organization of the E12.5 placenta. Markers of different cell

8 types used in this study are indicated.

9 b) Experimental design of tetraploid complementation assay. Tetraploid host embryos are

10 generated by electro-fusing embryos at the 2-cell stage. A small clump of ESCs are aggregated

11 with tetraploid host embryos to generate a chimera. Chimeric placentas are analyzed at E12.5.

12 c) Examples of chimeric E12.5 placentas generated with H2B-Gfp expressing tetraploid host

13 embryo and wild-type ESCs (left panel) and wild-type tetraploid host embryo and H2B-Gfp

14 expressing ESCs (right panel). Placenta sections are immuno-stained for Gfp and Tfap2c, which

15 labels all trophoblast cell types or CD31, which labels fetal (embryo-derived) endothelial cells.

16 Images shown at different magnifications. Scale bars: $500 \mu \mathrm{m}(4 \mathrm{x}), 200 \mu \mathrm{m}(7 \mathrm{x}), 50 \mu \mathrm{m}(40 \mathrm{x})$.

17 Sections from 4 chimeric placentas were analyzed.

18 d) Example of chimeric E12.5 placentas generated with wild-type host embryo (diploid) and

19 H2B-Gfp expressing L-EPSCs. Placenta sections are immuno-stained for Gfp and Tfap2c or

20 CD31. Images shown at different magnifications. Scale bars: $500 \mu \mathrm{m}(4 \mathrm{x}), 200 \mu \mathrm{m}(7 \mathrm{x}), 50 \mu \mathrm{m}$

21 (40x). Sections from 4 chimeric placentas were analyzed.

\section{References} embryonic stem cell research. Nat Rev Genet 7, 319-327, doi:10.1038/nrg1827 (2006). 
2 Rossant, J. Investigation of the determinative state of the mouse inner cell mass. II. The 2 fate of isolated inner cell masses transferred to the oviduct. J Embryol Exp Morphol 33, 3 991-1001 (1975).

43 Tarkowski, A. K., Suwinska, A., Czolowska, R. \& Ozdzenski, W. Individual blastomeres of 16- and 32-cell mouse embryos are able to develop into foetuses and mice. Dev Biol 348, 190-198, doi:10.1016/j.ydbio.2010.09.022 (2010).

4 Posfai, E. et al. Position- and Hippo signaling-dependent plasticity during lineage segregation in the early mouse embryo. Elife 6, doi:10.7554/eLife.22906 (2017).

5 Cockburn, K. \& Rossant, J. Making the blastocyst: lessons from the mouse. J Clin Invest 120, 995-1003, doi:10.1172/JCI41229 (2010).

6 Kwon, G. S., Viotti, M. \& Hadjantonakis, A. K. The endoderm of the mouse embryo arises by dynamic widespread intercalation of embryonic and extraembryonic lineages. Dev Cell 15, 509-520, doi:10.1016/j.devcel.2008.07.017 (2008).

7 Nowotschin, S. et al. The emergent landscape of the mouse gut endoderm at single-cell resolution. Nature 569, 361-367, doi:10.1038/s41586-019-1127-1 (2019).

8 Evans, M. J. \& Kaufman, M. H. Establishment in culture of pluripotential cells from mouse embryos. Nature 292, 154-156, doi:10.1038/292154a0 (1981).

9 Martin, G. R. Isolation of a pluripotent cell line from early mouse embryos cultured in medium conditioned by teratocarcinoma stem cells. Proc Natl Acad Sci U S A 78, 76347638, doi:10.1073/pnas.78.12.7634 (1981).

10 Tanaka, S., Kunath, T., Hadjantonakis, A. K., Nagy, A. \& Rossant, J. Promotion of trophoblast stem cell proliferation by FGF4. Science 282, 2072-2075, doi:10.1126/science.282.5396.2072 (1998).

11 Kunath, T. et al. Imprinted X-inactivation in extra-embryonic endoderm cell lines from mouse blastocysts. Development 132, 1649-1661, doi:10.1242/dev.01715 (2005).

12 Ying, Q. L. et al. The ground state of embryonic stem cell self-renewal. Nature 453, 519523, doi:10.1038/nature06968 (2008).

13 Niakan, K. K., Schrode, N., Cho, L. T. \& Hadjantonakis, A. K. Derivation of extraembryonic endoderm stem (XEN) cells from mouse embryos and embryonic stem cells. Nat Protoc 8, 1028-1041, doi:10.1038/nprot.2013.049 (2013).

14 Cho, L. T. et al. Conversion from mouse embryonic to extra-embryonic endoderm stem cells reveals distinct differentiation capacities of pluripotent stem cell states. Development 139, 2866-2877, doi:10.1242/dev.078519 (2012).

15 Papaioannou, V. E., McBurney, M. W., Gardner, R. L. \& Evans, M. J. Fate of teratocarcinoma cells injected into early mouse embryos. Nature 258, 70-73, doi:10.1038/258070a0 (1975).

16 Gardner, R. L. \& Rossant, J. Investigation of the fate of 4-5 day post-coitum mouse inner cell mass cells by blastocyst injection. J Embryol Exp Morphol 52, 141-152 (1979).

17 Niakan, K. K. et al. Sox17 promotes differentiation in mouse embryonic stem cells by directly regulating extraembryonic gene expression and indirectly antagonizing selfrenewal. Genes Dev 24, 312-326, doi:10.1101/gad.1833510 (2010).

1 Canham, M. A., Sharov, A. A., Ko, M. S. \& Brickman, J. M. Functional heterogeneity of embryonic stem cells revealed through translational amplification of an early endodermal transcript. PLoS Biol 8, e1000379, doi:10.1371/journal.pbio.1000379 (2010). 
19 Beddington, R. S. \& Robertson, E. J. An assessment of the developmental potential of

20 Macfarlan, T. S. et al. Embryonic stem cell potency fluctuates with endogenous retrovirus activity. Nature 487, 57-63, doi:10.1038/nature11244 (2012).

21 Rodriguez-Terrones, D. et al. A molecular roadmap for the emergence of early-embryoniclike cells in culture. Nat Genet 50, 106-119, doi:10.1038/s41588-017-0016-5 (2018).

22 Niwa, H. et al. Interaction between Oct3/4 and $\mathrm{Cdx} 2$ determines trophectoderm differentiation. Cell 123, 917-929, doi:10.1016/j.cell.2005.08.040 (2005).

$23 \mathrm{Ng}$, J. H., Heng, J. C., Loh, Y. H. \& Ng, H. H. Transcriptional and epigenetic regulations of embryonic stem cells. Mutat Res 647, 52-58, doi:10.1016/j.mrfmmm.2008.08.009 (2008).

24 Kuckenberg, P. et al. The transcription factor TCFAP2C/AP-2gamma cooperates with CDX2 to maintain trophectoderm formation. Mol Cell Biol 30, 3310-3320, doi:10.1128/MCB.01215-09 (2010).

25 Cambuli, F. et al. Epigenetic memory of the first cell fate decision prevents complete ES cell reprogramming into trophoblast. Nat Commun 5, 5538, doi:10.1038/ncomms6538 (2014).

26 Yamanaka, S. \& Takahashi, K. [Induction of pluripotent stem cells from mouse fibroblast cultures]. Tanpakushitsu Kakusan Koso 51, 2346-2351 (2006).

27 Benchetrit, H. et al. Direct Induction of the Three Pre-implantation Blastocyst Cell Types from Fibroblasts. Cell Stem Cell 24, 983-994 e987, doi:10.1016/j.stem.2019.03.018 (2019).

28 Tesar, P. J. et al. New cell lines from mouse epiblast share defining features with human embryonic stem cells. Nature 448, 196-199, doi:10.1038/nature05972 (2007).

29 Brons, I. G. et al. Derivation of pluripotent epiblast stem cells from mammalian embryos. Nature 448, 191-195, doi:10.1038/nature05950 (2007).

30 Hall, J. et al. Oct4 and LIF/Stat3 additively induce Kruppel factors to sustain embryonic stem cell self-renewal. Cell Stem Cell 5, 597-609, doi:10.1016/j.stem.2009.11.003 (2009).

31 Guo, G. et al. Klf4 reverts developmentally programmed restriction of ground state pluripotency. Development 136, 1063-1069, doi:10.1242/dev.030957 (2009).

32 Morgani, S. M. et al. Totipotent embryonic stem cells arise in ground-state culture conditions. Cell Rep 3, 1945-1957, doi:10.1016/j.celrep.2013.04.034 (2013).

33 Abad, M. et al. Reprogramming in vivo produces teratomas and iPS cells with totipotency features. Nature 502, 340-345, doi:10.1038/nature12586 (2013).

34 Choi, Y. J. et al. Deficiency of microRNA miR-34a expands cell fate potential in pluripotent stem cells. Science 355, doi:10.1126/science.aag1927 (2017).

35 Yang, J. et al. Establishment of mouse expanded potential stem cells. Nature 550, 393-397, doi:10.1038/nature24052 (2017).

Yang, Y. et al. Derivation of Pluripotent Stem Cells with In Vivo Embryonic and

41 Extraembryonic Potency. Cell 169, 243-257 e225, doi:10.1016/j.cell.2017.02.005 (2017). stem cells. Nat Cell Biol 19, 177-188, doi:10.1038/ncb3474 (2017).

38 Gao, X. et al. Establishment of porcine and human expanded potential stem cells. Nat Cell Biol 21, 687-699, doi:10.1038/s41556-019-0333-2 (2019). 
39 Li, R. et al. Generation of Blastocyst-like Structures from Mouse Embryonic and Adult Cell Cultures. Cell 179, 687-702 e618, doi:10.1016/j.cell.2019.09.029 (2019).

40 Sozen, B. et al. Self-Organization of Mouse Stem Cells into an Extended Potential Blastoid. Dev Cell 51, 698-712 e698, doi:10.1016/j.devcel.2019.11.014 (2019).

41 Deng, Q., Ramskold, D., Reinius, B. \& Sandberg, R. Single-cell RNA-seq reveals dynamic, random monoallelic gene expression in mammalian cells. Science 343, 193-196, doi:10.1126/science.1245316 (2014).

42 Mohammed, H. et al. Single-Cell Landscape of Transcriptional Heterogeneity and Cell Fate Decisions during Mouse Early Gastrulation. Cell Rep 20, 1215-1228, doi:10.1016/j.celrep.2017.07.009 (2017).

43 Chen, G. et al. Single-cell analyses of X Chromosome inactivation dynamics and pluripotency during differentiation. Genome Res 26, 1342-1354, doi:10.1101/gr.201954.115 (2016).

44 Aibar, S. et al. SCENIC: single-cell regulatory network inference and clustering. Nat Methods 14, 1083-1086, doi:10.1038/nmeth.4463 (2017).

45 Van de Sande, B. et al. A scalable SCENIC workflow for single-cell gene regulatory network analysis. Nat Protoc 15, 2247-2276, doi:10.1038/s41596-020-0336-2 (2020).

46 Rugg-Gunn, P. J., Cox, B. J., Ralston, A. \& Rossant, J. Distinct histone modifications in stem cell lines and tissue lineages from the early mouse embryo. Proc Natl Acad Sci US A 107, 10783-10790, doi:10.1073/pnas.0914507107 (2010).

47 Murray, A., Sienerth, A. R. \& Hemberger, M. Plet1 is an epigenetically regulated cell surface protein that provides essential cues to direct trophoblast stem cell differentiation. Sci Rep 6, 25112, doi:10.1038/srep25112 (2016).

Rivron, N. C. et al. Blastocyst-like structures generated solely from stem cells. Nature 557, 106-111, doi:10.1038/s41586-018-0051-0 (2018).

49 Kime, C. et al. Induced 2C Expression and Implantation-Competent Blastocyst-like Cysts from Primed Pluripotent Stem Cells. Stem Cell Reports 13, 485-498, doi:10.1016/j.stemcr.2019.07.011 (2019).

Pijuan-Sala, B. et al. A single-cell molecular map of mouse gastrulation and early organogenesis. Nature 566, 490-495, doi:10.1038/s41586-019-0933-9 (2019).

Davie, K. et al. A Single-Cell Transcriptome Atlas of the Aging Drosophila Brain. Cell 174, 982-998 e920, doi:10.1016/j.cell.2018.05.057 (2018).

52 Suwinska, A., Czolowska, R., Ozdzenski, W. \& Tarkowski, A. K. Blastomeres of the mouse embryo lose totipotency after the fifth cleavage division: expression of $\mathrm{Cdx} 2$ and Oct4 and developmental potential of inner and outer blastomeres of 16- and 32-cell embryos. Dev Biol 322, 133-144, doi:10.1016/j.ydbio.2008.07.019 (2008).

53 Strnad, P. et al. Inverted light-sheet microscope for imaging mouse pre-implantation development. Nat Methods 13, 139-142, doi:10.1038/nmeth.3690 (2016).

54 Strumpf, D. et al. Cdx2 is required for correct cell fate specification and differentiation of trophectoderm in the mouse blastocyst. Development 132, 2093-2102, doi:10.1242/dev.01801 (2005).

55 Home, P. et al. GATA3 is selectively expressed in the trophectoderm of peri-implantation embryo and directly regulates Cdx2 gene expression. J Biol Chem 284, 28729-28737, doi:10.1074/jbc.M109.016840 (2009).

56 Ralston, A. et al. Gata3 regulates trophoblast development downstream of Tead4 and in parallel to Cdx2. Development 137, 395-403, doi:10.1242/dev.038828 (2010). 
57 Brulet, P., Babinet, C., Kemler, R. \& Jacob, F. Monoclonal antibodies against trophectoderm-specific markers during mouse blastocyst formation. Proc Natl Acad Sci U $S$ A 77, 4113-4117, doi:10.1073/pnas.77.7.4113 (1980).

58 Avilion, A. A. et al. Multipotent cell lineages in early mouse development depend on SOX2 function. Genes Dev 17, 126-140, doi:10.1101/gad.224503 (2003).

59 Watson, E. D. \& Cross, J. C. Development of structures and transport functions in the mouse placenta. Physiology (Bethesda) 20, 180-193, doi:10.1152/physiol.00001.2005 (2005).

60 Dumollard, R., Carroll, J., Duchen, M. R., Campbell, K. \& Swann, K. Mitochondrial function and redox state in mammalian embryos. Semin Cell Dev Biol 20, 346-353, doi:10.1016/j.semcdb.2008.12.013 (2009).

61 Whittington, N. C. \& Wray, S. Suppression of Red Blood Cell Autofluorescence for Immunocytochemistry on Fixed Embryonic Mouse Tissue. Curr Protoc Neurosci 81, 228 21-22 28 12, doi:10.1002/cpns.35 (2017).

62 Sferruzzi-Perri, A. N., Higgins, J. S., Vaughan, O. R., Murray, A. J. \& Fowden, A. L. Placental mitochondria adapt developmentally and in response to hypoxia to support fetal growth. Proc Natl Acad Sci U S A 116, 1621-1626, doi:10.1073/pnas.1816056116 (2019).

63 Albers, R. E. et al. Gestational differences in murine placenta: Glycolytic metabolism and $\begin{array}{llll}\text { pregnancy } & \text { parameters. } & \text { 107, }\end{array}$ doi:10.1016/j.theriogenology.2017.10.049 (2018).

64 Tanaka, M., Hadjantonakis, A. K. \& Nagy, A. Aggregation chimeras. Combining ES cells, diploid and tetraploid embryos. Methods Mol Biol 158, 135-154, doi:10.1385/1-59259220-1:135 (2001).

65 Nagy, A., Gertsenstein, M., Vintersten, K. \& Behringer, R. Assembling Aggregates between Embryonic Stem (ES) Cells and Tetraploid Embryos. CSH Protoc 2006, doi:10.1101/pdb.prot4426 (2006).

66 Tomoda, K. et al. Reprogramming Epiblast Stem Cells into Pre-Implantation Blastocyst Cell-like Cells. bioRxiv, 2020.2009.2029.318279, doi:10.1101/2020.09.29.318279 (2020).

67 Tremble, K. et al. Sox2 modulation increases naïve pluripotency plasticity. bioRxiv, 2020.2001.2014.906933, doi:10.1101/2020.01.14.906933 (2020).

68 Yachie-Kinoshita, A. et al. Modeling signaling-dependent pluripotency with Boolean logic to predict cell fate transitions. Mol Syst Biol 14, e7952, doi:10.15252/msb.20177952 (2018).

69 Kurek, D. et al. Endogenous WNT signals mediate BMP-induced and spontaneous differentiation of epiblast stem cells and human embryonic stem cells. Stem Cell Reports 4, 114-128, doi:10.1016/j.stemcr.2014.11.007 (2015). 


\section{Methods}

\section{ESC and EPSC culture}

Naïve mouse ESCs and D-EPSCs were cultured in a base medium of N2B27 prepared as follows: 1:1 ratio of DMEM/F12 (ThermoFisher Scientific 21331020) and Neurobasal (ThermoFisher Scientific 21103049); 1x N2 supplement (ThermoFisher Scientific 17502001) or 1xNDiff Neuro2 supplement (Gibco, 17502048); 1x B27 supplement minus vitamin A (ThermoFisher Scientific 12587-010 or 17504044); 1x Glutamax (ThermoFisher Scientific 35050061); $0.1 \mathrm{mM} \beta$-mercaptoethanol (ThermoFisher Scientific 21985023). For best results, this base media was further supplemented with $1-5 \%$ knockout serum replacement (KSR; ThermoFisher Scientific 10828028) as described ${ }^{71,36}$. KSR was not used during culture of tdTomato D-EPSCs, as it was indicated as optional in the original publication ${ }^{36}$. Both naïve ESCs and D-EPSCs were cultured at $20 \% \mathrm{O} 2$ and $5 \% \mathrm{CO} 2$ at $37^{\circ} \mathrm{C}$ on mitomycin $\mathrm{C}(\mathrm{MMC}$; Sigma-Aldrich M0503) inactivated mouse embryonic fibroblast (MEF) feeder cells (approx 30,000 cells/cm2). E12.5 DR4 MEFs were routinely plated on $0.2 \%$ gelatin-coated plates in ESC base media prepared as follows: DMEM (ThermoFisher Scientific 11960069); 15\% foetal bovine serum (FBS; Wiscent); 1x Glutamax (ThermoFisher Scientific 35050061); 1x nonessential amino acids (NEAA; ThermoFisher Scientific 11140-050); 1 mM sodium pyruvate (ThermoFisher Scientific 11360070); $0.1 \mathrm{mM} \beta$-mercaptoethanol (ThermoFisher Scientific 21985023 or Merck, M3148 SIGMA). MEF plates were used within 1 week and washed with DPBS (ThermoFisher Scientific 14190250) prior to plating of ESC/D-EPSCs in appropriate media.

For naïve ESC culture, N2B27 (1-5\% KSR) base media was supplemented with $1 \mu \mathrm{M}$ PD0325901 (Tocris 4192); $3 \mu \mathrm{M} \mathrm{CHIR99021} \mathrm{(Tocris} \mathrm{4423);} \mathrm{and} 1000 \mathrm{U} / \mathrm{mL}$ mouse LIF (generated in-house). For D-EPSC culture, N2B27 1-5\% KSR base media was supplemented with 1x NEAA (ThermoFisher Scientific 11140-050); $10 \mathrm{ng} / \mathrm{mL}$ recombinant human LIF (hLIF; Peprotech 300-05); $3 \mu \mathrm{M} \mathrm{CHIR99021} \mathrm{(Tocris} \mathrm{4423);} 2 \mu \mathrm{M}$ Dimethindene maleate (Tocris \#1425); and 2 M Minocycline Hydrochloride (Santa Cruz \#sc-203339). Media was changed 
daily for both ESCs and D-EPSCs, with single-cell passaging every 2-3 days using accutase (ThermoFisher Scientific A1110501) at split ratios between 1:5 and 1:12.

L-EPSCs were cultured in a base media prepared as follows: DMEM/F12 (ThermoFisher Scientific 21331020); 20\% KSR (ThermoFisher Scientific 10828028); 1x Glutamax (ThermoFisher Scientific 35050061) (or DMEM/F12 (Gibco, 13320074), 20\% KnockOut Serum Replacement (KSR, Gibco, 10828028), $2.25 \mathrm{mM}$ L-glutamine); 1x NEAA (ThermoFisher Scientific 11140-050); $0.1 \mathrm{mM} \beta$-mercaptoethanol (ThermoFisher Scientific 21985023 or Merck, M3148 SIGMA). This base media was supplemented with $10 \mathrm{ng} / \mathrm{mL} \mathrm{hLIF}$ (Peprotech 300-05) or $1000 \mathrm{U} / \mathrm{ml}$ homemade mouse Lif; $1 \mu \mathrm{M}$ PD0325901 (Tocris 4192 or Axon 1408); $3 \mu \mathrm{M}$ CHIR99021 (Tocris 4423, or Axon 1386); 4 M TCS JNK 60 (Tocris 3222);

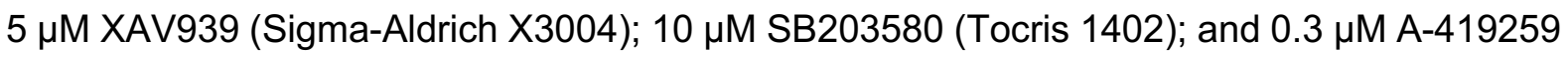
(Santa Cruz sc-36109; or Tocris 39142). L-EPSCs were cultured at 20\% O2 and 5\% CO2 at $37^{\circ} \mathrm{C}$ on MMC-inactivated SNL76/7 feeder cells or MMC-inactivated MEF feeders $(\sim 50-80,000$ cells $/ \mathrm{cm}^{2}$ ). SNL feeders were plated on $0.2 \%$ gelatin-coated plates in ES base media (described above). SNL plates were used within 3-4 days and washed with DPBS prior to plating L-EPSCs. Media was changed daily, with single-cell passaging every 3 days using accutase at split ratios between 1:3 and 1:12.

\section{Cell line generation}

H2B-eGfp ESCs (ICR) were derived directly from E3.5 blastocysts (JAX 006069 backcrossed to ICR in-house ${ }^{72}$ ). Briefly, single E3.5 blastocysts were transferred into a well containing 100 $\mu \mathrm{L}$ 2iLif media (96-well plate pre-coated with E12.5 DR4 MEFs). Embryos were left undisturbed for approx 48 hours, at which point additional $100 \mu \mathrm{L}$ 2iLif media was added per well. The top $100 \mu \mathrm{L}$ of media was then changed every two days until the outgrowth reached passaging size ( $~ 5-7$ days). Outgrowths were passaged using $50 \mu \mathrm{L}$ accutase per well and plated into a new 96-well MEF plate. Cells were then passaged as described above until expanded for morphological assessment and cryopreservation of selected clones. 
R1-mScarlet-NLS ESCs were generated from R1-ESCs $\left(129 \times 1 \times 129 S 1 ;^{73}\right)$ by transfection (Lipofectamine 2000; ThermoFisher Scientific 11668027) with PiggyBac CAG-mScarlet-NLS construct and isolation of single clones.

Wild-type TSCs were TS-F4 (ICR) described previously ${ }^{74}$. Elf5-2A-mCherry TSCs were generated by transfection (JetPRIME; Polyplus 114-07) of TS-F4 cells with a vector expressing Cas9 and gRNA targeting proximal to Elf5 stop codon. Donor construct containing desired insert (GSG-P2A-SV40-NLS) and selection cassette (SV40::NeoR) flanked by homology arms was co-transfected. Cells were selected with 100-200 $\mu \mathrm{g} / \mathrm{mL}$ G418 (ThermoFisher Scientific 10131027) and correctly targeted clones identified by PCR genotyping and Sanger sequencing. Floxed selection cassette was subsequently removed by transfection with Cre and desired clones identified by PCR genotyping and Sanger sequencing. Heterozygous Elf5-2A-mCherry TSC clones were used in this study and faithful overlap between Elf5 and mCherry protein validated by immunostaining.

5ECER4G20 eGfp-Cdx2-ER (iCdx2) ESCs (129/Ola background) were a kind gift from $\mathrm{H}$. Niwa $^{22}$. These cells constitutively express eGfp and are heterozygous for Oct4 (Pou5F1). Heterozygous Elf5-2A-mCherry iCdx2 ESCs were generated as above, except for the use of SV40::HygroR selection cassette and Lipofectamine based transfection. These cells were used at passage $x+24$, where $x$ is the unknown passage number upon receipt of the cells.

\section{Conversion to EPSC conditions}

To convert naïve ESCs into L-EPSC conditions: (i) ESC lines derived from E4 blastocysts of C57BLx6J x Cast/Eij mouse crosses (Chen et al. $\left.(2016)^{43}(10), 1342-1354\right)$ at passage P14 were used for L-EPSC conversion in Figure 1A ; (ii) H2B-eGfp ESCs (ICR) used for chimera assays were converted at passage 12; and (iii) R1-mScarlet-NLS ESCs (129X1 x 129S1) used for chimera assays were converted at passage 25. tdTomato expressing C1-12 D-EPSCs (C57 x 129 F1 hybrid) were a kind gift from Dr. Jun Wu (UT Southwestern). These cells were orginally derived from blastocysts (B6.Tg(Sox2-cre)1Amc/J $\quad x \quad$ B6.Cg- 
Gt(ROSA)26Sortm14(CAG-Tdtomato)Hze/J) in D-EPSC conditions in the laboratory of Dr. Hongkui Deng and transferred to the Wu laboratory, where they underwent minimal expansion before being shipped to us at passage $26^{36}$. Experiments with E4.5 chimeras were performed at $26+7$ and E6.25 chimeras at $26+12$ passages using these cells. Cells were passaged and plated at a range of low cell densities (1-3,000 cells/cm2) on either MMC-inactivated E12.5 WT or DR4 MEFs or SNL feeders in their original media. The following day the media was changed to D-EPSC or L-EPSC conditions, respectively. The cells were passaged when approaching confluence (4-5 days) at a range of split ratios (e.g. 1:3, 1:5, 1:8). D-EPSCs and L-EPSCs converted in our laboratory were cultured for at least 5 passages ( 2 weeks) prior to use in chimaera or in vitro potency experiments.

\section{TSC culture}

TSCs were cultured in standard conditions: RPMI-1640 (Sigma-Aldrich R0883); 20\% FBS (Wiscent); 1x Glutamax (ThermoFisher Scientific 35050061); $1 \mathrm{mM}$ sodium pyruvate (ThermoFisher Scientific 11360070); $0.1 \mathrm{mM} \beta$-mercaptoethanol (ThermoFisher Scientific 21985023). This TS base media was supplemented with 25 ng/mL FGF4 (R\&D Systems 235F4) and $1 \mu \mathrm{g} / \mathrm{ml}$ heparin (Sigma-Aldrich H3149). TSCs were routinely cultured on MEFs plated on $0.2 \%$ gelatin-coated plates (approx 30,000 cells $/ \mathrm{cm}^{2}$ ). Medium was changed every 1-2 days and cells passaged before reaching confluency (every 3-4 days) at split ratios between $1: 5$ and $1: 12$.

\section{ESC-to-TSC reprogramming}

For in vitro testing of EPSC potency, iCdx2 Elf5-2A-mCherry ESCs were converted to either D-EPSC or L-EPSC conditions as described above. To initiate ESC-to-TSC reprogramming, 50-200 cells $/ \mathrm{cm}^{2}$ were plated onto low density E12.5 DR4 MEFs $\left(\sim 10,000\right.$ cells $/ \mathrm{cm}^{2}$ on gelatincoated plate) in their original media. The following day the media was changed to TSC media (+Fgf4/Heparin) with or without $1 \mu \mathrm{g} / \mathrm{mL}$ 4-hydroxytamoxifen (Sigma-Aldrich H7904) to induce 
Cdx2. Media was changed daily, and the degree of TSC reprogramming assessed by flow cytometry on day 6.

For ESC-to-TSCs differentiation followed by RT-qPCR, L-EPSCs and ESCs were gradually feeder-depleted by passaging every two days with $0.1 \%$ gelatin (porcine skin, $0.1 \% \mathrm{~g} / \mathrm{v}$ final, Sigma, G2500) and a feeder percentage of $100 \%, 75 \%, 50 \%$ to $0 \%$ at every passage in LEPSCM and 2iLif, respectively. After complete feeder removal, the cells were cultured at a density of $1 \times 10^{5}$ cells on gelatin-coated culture 6 well plates in TSC medium (as above). The cell culture medium was refreshed every two days and cells were collected every three days from D0 to D12 for RT-qPCR analysis of TSC marker genes expression using the primers listed in Supplementary Table 6.

\section{Flow cytometry}

Flow cytometry assay was designed to assess differential protein levels of TSC surface markers $\mathrm{CD}^{4} \mathrm{O}^{74}$ and Plet $1^{47}$, as well as TSC transcription factor Elf5 $5^{23}$ using a reporter cell line. Titration experiments were carried out to optimize staining against CD40 (1:20; BD Biosciences 740700) as well as Plet1 (1:200; Nordic MUbio MUB1512P conjugated to BioRad ReadiLink 633/655 1351005). Single-cell suspensions for ESCs and EPSCs undergoing ES-to-TSC reprogramming as well as control cell lines (ESC/EPSC day 0 samples; wild-type TSCs and Elf5-2A-mCherry TSCs) were generated using $0.25 \%$ trypsin (ThermoFisher Scientific 25200072). Cells were incubated with conjugated antibody mix in PBS/2\% FBS for 60 min on ice. Post-staining, cells were washed twice with PBS/2\% FBS prior to resuspension in PBS/2\% FBS supplemented with $0.5 \mu \mathrm{m}$ Sytox blue (ThermoFisher Scientific S11348) to assess cell viability. Cells were transferred to flow tubes with $40 \mu \mathrm{m}$ cell strainer lid and analyzed using BD LSR II at the SickKids Flow Cytometry facility. For ES-to-TSC reprogramming samples, at least 100,000 events were recorded and analyzed using FlowJo software (version 10). Gates were drawn based on fluorescence minus one (FMO) controls.

\section{Bulk RNA sequencing library preparation}


Total RNA was isolated from 2iLif ESCs and L-EPSCs reprogramming intermediates at indicated days using TRIzol reagent according to the manufacturer's protocol. $4 \mu \mathrm{g}$ of total RNA was used to generate libraries using of stranded poly(A) mRNA-Seq library with the KAPA stranded mRNA Library prep kit (KAPA Biosystems, KK8421). Libraries concentration was quantified with Qubit dsDNA HS (High Sensitivity) Assay Kit (Invitrogen, Q32854), and the final pool was generated by combining individual libraries in equimolar ratio. Libraries were sequenced on an Illumina HiSeq 4000 instrument (Illumina) with 51 bp reads and read depth ranging $\sim 33 \mathrm{mln}$ reads. Sequencing reads were mapped to $\mathrm{mm} 10$ reference genome using STAR $2.5 .3 a^{75}$. On average, $77,16 \%$ of reads were uniquely mapped and only those were kept for further analyses. Subsequently, the featureCounts function from the R Bioconductor package "Rsubread" (version 1.5.2) ${ }^{76}$ was used to assign mapped reads to genomic features. DEG analysis was done using DESeq2 and filtered by adjusted $p$ value $<0.05$. Pathway enrichment analysis was performed using panther with multiple comparisons correction.

\section{Bulk RNA sequencing analysis}

Processing raw read counts was performed as described $\mathrm{in}^{77}$. Briefly, the DESeq2 package and the associated protocol $^{78}$ was used. Only genes that express at least 10 reads in total across all libraries were retained. PCA was performed using plotPCA function from the DESeq2 package with input of top 500 most variable genes after rlog transformation. Unless mentioned otherwise, gene expression was presented as log2 values after size-factor normalization for the differences in library size (DESeq2).

\section{Integration of bulk RNA-seq data with published single-cell RNA-seq data}

Principal component analysis of top 500 most variable genes. Single-cell data from published datasets (Deng et al. 2014 ${ }^{41}$; Posfai et al. 20174; Yang et al. $2017^{36}$ ) were processed together with samples from this study. The reads from single-cell samples were averaged within the same embryonic timepoint. Subsequently, all samples were normalized together for the library size using size-factor normalization in the DESeq2 package. 
For the comparison of 4-cell stage markers between different L-EPSC conversion timepoints, published bulk RNA-seq data (Yang et al. 201735, Yang et al. 2017 ${ }^{36}$ ) and scRNA-seq data (Deng et al. 2014 ${ }^{41}$; Yang et al. $2017^{35}$ ) were used. 4-cell stage markers were defined by performing differential gene expression analysis between cells from each stage of embryonic development using single-cell data from Deng et al. $2014^{41}$ and FindAllMarkers function in Seurat package. Top 500 significantly different $(F D R<0,05)$ were retained. Subsequently, these markers were used for the comparison with the averaged single-cell data.

\section{Library preparation for single-cell RNA sequencing}

Single-cells from stem cells were sorted by FACS into 384 well plates containing lysis and RT, whereas embryo cells were manually picked and directly dispensed into lysis buffer containing RT. The current study generated cDNA libraries using the Smart-seq2 protocol, as previously described ${ }^{79,80}$.

\section{Single-cell RNA sequencing data pre-processing and quality control}

Smart-Seq2 read files (E2.5 and E4.5 embryos, and three pluripotent stem cell conditions: ESCs cultured in 2iLif, L-EPSCs, and D-EPSCs) were mapped to the mouse reference genome (mm10) using STAR aligner ${ }^{75}$ and only uniquely mapped reads were used for expression level estimation as reads per kilobase of gene model and million mappable reads (RPKMs) using RefSeq annotation and previously established pipeline ${ }^{81}$. Cells were qualityfiltered with minimum cut-off of 500 expressed genes per cell and Spearman's correlation greater than $60 \%$ between cells. Pre-processed gene expression matrices were downloaded as provided by Deng et al., $2014^{41}$ (GSE45719), Posfai et al., 20174 (GSE84892), Mohammed et al., $2017^{42}$ (GSE100597), Chen et al., 2016 ${ }^{43}$ (GSE74155), Li et al., 201939 (GSE135289, GSE135701), Yang et al., 2017 35 (ERP005641), Pijuan-Sala et al., $2019^{50}$ (https://github.com/MarioniLab/EmbryoTimecourse2018), Sozen et al., 201940 (GSE134240). 


\section{Single-cell gene expression analysis of merged datasets}

Analysis of the filtered data was conducted in $\mathrm{R}$ version 3.6.1 using Seurat suite version 3.1.0. ${ }^{82}$. Integration of single-cell data was performed using Seurat's canonical correlation analysis (CCA) integration tool; datasets were scaled and log-transformed before selection of 2000 most variable genes which were used to compute principal component analysis (PCA). Manifold approximation and projection (UMAP) coordinates were calculated using the top 14 PCs. Seurat's FeaturePlot function was used to demonstrate individual gene expression on UMAP embedding. Merged datasets were clustered, annotated, then downsampled prior to CCA integration. Correlation matrixes were constructed using corrplot v0.84. Clustering of pre-implantation lineages was performed using Seurat's shared nearest neighbor (SNN) algorithm implemented in FindClusters function. Differential expression analysis was performed using Wilcoxon's rank sum test using a minimum cutoff of 0.45 average log fold change.

\section{Gene Regulatory Network inference}

Gene regulatory networks were inferred using pySCENIC (0.9.15; python implementation of SCENIC) ${ }^{44,45}$ in Python version 3.6.9. First, raw expression data were normalized by dividing feature counts of each cell by the total counts for that cell and multiplying by a factor of 10000 followed by $\log 1 p$ transformation. Subsequently, normalized counts were used to generate coexpression modules using GRNboost2 algorithm ${ }^{83}$ implemented in arboreto package $(v 0.1 .3)^{84}$. Next, gene regulatory networks were inferred using pySCENIC (with default parameters and $\mathrm{mm} 10$ _refseq-r80_10kb_up_and_down_tss.mc9nr and mm10_refseqr80_500bp_up_and_100bp_down_tss.mc9nr motif collections) resulting in the matrix of AUCell values that represent the activity of each regulon in each cell. The resulting gene regulatory networks contained 451 regulons for merged datasets in Figure 1 and Supplementary Figure 2 and 333 regulons for datasets in Figure 3, Supplementary Figures 4 and 5 and have been added to the loom files which can be downloaded from: https://github.com/pasquelab/totipotency and browsed interactively by uploading the loom file 
on the SCope platform, www.scope.aertslab.org. The loom files contain non-integrated gene expression and regulon data.

For generating UMAP plots based on gene regulatory information in Supplementary Figure 3B and Supplementary Figure 4A, the AUCell matrix was split by the dataset of origin and integrated using Seurat's canonical correlation analysis (CCA) integration tool. Anchors for integration were found using FindlntegrationAnchors function with default parameters and dims $=1: 20$, and data was integrated across all features. SCENIC-based UMAP were constructed using runUMAP function with default parameters except for dims $=25$ and min.dist $=0.35$ in Supplementary Figure 3B and dims $=25$ and min dist $=0.25$ in Supplementary Figure $4 \mathrm{~A}$.

The list of target genes were downloaded from the loom file through the SCope platform.

\section{Gene Set Enrichment Analysis}

Gene Set Enrichment Analysis was performed using the java-based GSEA (4.0.3; http://www.broadinstitute.org/gsea/downloads.jsp) application, using 'weighted' enrichment statistic. Signatures of mouse pre-implantation embryo stages were defined using single-cell RNA-seq data from Deng et al., $2014^{41}$. For each signature, after size-factor normalization in DESeq2, the top 500 genes that were more expressed on average than in other stages were selected (Supplemental file 5). GSEA was performed using count matrices normalized for the library size.

For signature used in Figure 1D, Deng et al., $2014{ }^{41}$ single-cell RNA-seq data was used after processing in Seurat package. After normalizing and scaling the data, differentially expressed genes between all stages were identified using FindAllMarkers() function with logfc.threshold parameter set to 0.25 and top 500 markers were retained in the signature (Supplemental file 4).

\section{Mouse lines and embryos}


ICR (breeding stock from Charles River, Montreal, Canada), DsRed ${ }^{85}, H 2 B-G f p^{72}$ mouse lines were used in this study. Embryos were collected at appropriate time points from 5-8 week old hormone-primed (5 IU each, pregnant mare serum gonadotropin (Sigma) and human chorionic gonadotropin (Sigma), 48 hours apart) and mated females. Males used for mating were 8-20 week old ICRs. If not immediately used, embryos were cultured in small drops of KSOM supplemented with amino acids (EMD Milipore) under mineral oil (Zenith Biotech, Guilford, CT) at $37^{\circ} \mathrm{C}$, with $5 \% \mathrm{CO} 2$ for specified times. Animal work was carried out following Canadian Council on Animal Care Guidelines for Use of Animals in Research and Laboratory Animal Care under protocols approved by The Centre for Phenogenomics Animal Care Committee $(20-0026 \mathrm{H})$ or after approval by the Southern Stockholm Animal Experimental Ethics Committee (DNR S-144-12) or after approval by the Animal Research Ethics Committee at KU Leuven (ECD_P003-2016). The study is compliant with all relevant ethical regulations regarding animal research. Laboratory mice were housed at $65-75^{\circ} \mathrm{F}$ with $40-$ $60 \%$ humidity, on a 12 light/12 dark cycle (6am/6pm).

\section{Generation, culture and isolation of chimeras using diploid or tetraploid hosts}

To isolate 8-cell stage blastomeres embryos from H2B-Gfp or DsRed mouse lines were harvested on the morning of day E2.5 by flushing the oviduct. The zona pellucida was removed using acid Tyrode's solution (Sigma, Oakville, Canada) and embryos were washed in M2. Dissociation was performed by incubating embryos in TrypLE Select (Gibco ${ }^{\mathrm{TM}}$, Thermo Fisher Scientific, Waltham, MA) for $3-6$ minutes at $37^{\circ} \mathrm{C}$ followed by pipetting through fine pulled glass capillaries. Individual cells were picked, washed in warm M2 and used as donor cells. For host embryos, E2.5 embryos were isolated and zona was removed in a similar way. To generate aggregation chimeras a single donor cell and a single host embryo were then brought together in a micro-well generated by pressing a blunt end needle into the bottom of a plastic tissue culture dish (Falcon ${ }^{\mathrm{TM}}$, Thermo Fisher Scientific) in drops of KSOM under oil. Such 
aggregation chimeras were either cultured for 48 hours (E4.5) or transferred into oviducts of pseudopregnant females on the following day and isolated either on day E6.25 or E12.5.

For generating chimeras with different stem cells (ESC, L-EPSC, D-EPSC), host embryos were generated as described above. A small clump (6-8 cells) of stem cells expressing H2BGfp were aggregated to each host embryo in a micro-well. Stem cell clumps were made by briefly trypsinizing the cells, inactivating or diluting trypsin with media and manually picking appropriate-sized clumps using a fine glass capillary. Chimeras were cultured or transferred into pseudopregnant females as above.

For tetraploid complementation experiments, embryos from either wild-type or H2B-Gfp mouse lines were isolated at the 2-cell stage (E1.5). Cells were electro-fused into one tetraploid cell using a Cell-fusion instrument, CF-150B pulse generator with $250 \mu \mathrm{m}$ electrode chamber (BLS Ltd, Hungary). The procedure is described in detail $\mathrm{in}^{86}$. Such tetraploid embryos were cultured for one day before using them as host embryos. In tetraploid complementation experiments, a small clump (6-8 cells) of ESCs either from a wild-type line or an H2B-Gfp expressing cell line was used. Aggregation, chimera culture and transfer were performed as before.

\section{Immunofluorescent staining of E4.5 and E6.25 chimeras}

Whole mount immunofluorescence staining of E4.5 embryos was performed as previously described ${ }^{4}$. Briefly, embryos were fixed in $4 \%$ paraformaldehyde at room temperature for 15 minutes, washed once in PBS containing 0.1\% Tween 20 (PBS-T) and permeabilized for 15 minutes in PBS $0.5 \%$ Triton X-100. E4.5 embryos were blocked in PBS-T with $2 \%$ BSA (Sigma) and 5\% normal donkey serum (Jackson ImmunoResearch Laboratories) at room temperature for 2 hours and E6.25 embryos were blocked in PBS-T with 10\% BSA (Sigma) and 5\% normal donkey serum (Jackson ImmunoResearch Laboratories) at room temperature for 8 hours or overnight at $4^{\circ} \mathrm{C}$. Primary and secondary antibodies were diluted in blocking solution. Staining was performed at room temperature for $\sim 2-5$ hours or overnight at $4^{\circ} \mathrm{C}$. Washes after primary and secondary antibodies were done three times in PBS-T. In the last 
washing step (15 minutes) 1:500 Hoechst 33258 (Invitrogen) was used to stain nuclei. E4.5 embryos were mounted in PBS in wells made with Secure Seal spacers (Molecular Probes ${ }^{\mathrm{TM}}$, Thermo Fisher Scientific) and placed between two cover glasses for imaging. E6.25 embryos were mounted in agarose plugs using $1 \%$ low melting agar (Sigma).

\section{Immunofluorescent staining of E12.5 placentas}

Placentas were dissected from pregnant females at E12.5, washed briefly in ice-cold PBS and fixed in $4 \%$ PFA overnight. Depending on the experiment, embryos and placentas were prescreened for contribution to individual compartments using a fluorescent stereomicroscope. The following day, placentas were embedded, frozen and sectioned $(10 \mu \mathrm{m})$ starting at the sagittal plane. Sections were blocked with $10 \%$ horse serum in PBS for 1-hr at room temperature and incubated overnight at $4^{\circ} \mathrm{C}$ with primary antibodies diluted with $5 \%$ horse serum in PBS. DsRed chimeric placentas were stained with anti-mCherry/Rfp antibody and H2B-Gfp chimeric placentas with anti-GFP. For detection of fetal endothelial cells, placentas were first subjected to antigen retrieval using $10 \mathrm{mM}$ citrate buffer, $\mathrm{pH} 6.0$ at $100^{\circ} \mathrm{C}$ for 10 minutes, then cooled, blocked, and co-stained with anti-CD34. The following day, sections were stained with the appropriate secondary antibodies for 1 -hr at room temperature, washed in PBS, and eventually counterstained with DAPI (Sigma). Sections exposed to secondary antibody alone were used as negative controls. Antibody specificity for mCherry/Rfp and Gfp were confirmed on non-chimeric placentas.

\section{Antibodies for immunofluorescence stainings}

Antibody information provided in Supplementary Table 7.

\section{Imaging}

Images of E4.5 embryos were acquired using a Zeiss Axiovert 200 inverted microscope equipped with a Hamamatsu C9100-13 EM-CCD camera, a Quorum spinning disk confocal scan head and Volocity acquisition software (PerkinElmer, version 6.3.0). Z-stacks were taken 
at $1 \mu \mathrm{m}$ intervals with a $20 \mathrm{x}$ air objective $(\mathrm{NA}=0.75)$. Images of $\mathrm{E} 6.25$ embryos were acquired using a Ziess Ligh Sheet Z1, with a pCO Edge 5.5 x 2 camera and Zeiss Zen Lightsheet 2014 acquisition software (version 9.1.0.268). Images were acquired using a 20x water immersion detection objective $(\mathrm{NA}=1.0)$. Z-stacks were taken at $1 \mu \mathrm{m}$ intervals. Images of E12.5 placentas were acquired using a Pannoramic 250 Flash II from 3DHistech.

\section{Statistics and reproducibility for imaging data}

For chimeric embryos analyzed by imaging, aggregations with each cell line, or totipotent embryo cell was performed once. For 2iLif, L-EPSC and D-EPSC chimeras at E4.5 and E6.25 two independent cell lines were used. For 8cell blastomere chimeras at E4.5 and E12.5 two independent mouse lines were used. For chimeras analyzed at E4.5 at least 10 chimeric embryos were analyzed, for chimeras analyzed at E6.25 at least 5 embryos were analyzed, and for E12.5 chimeric placentas at least 4 were analyzed. These numbers were chosen based on previous standards in the field. Numbers for each experiment are provided in the Source Data Fig. 4-6.

\section{Code Availability}

Codes pertaining to important analyses in this study are available from the Pasque lab Github webpage (https://github.com/pasquelab/totipotency).

\section{Data Availability}

All raw and processed sequencing data, as well as .loom files for visualization in SCope, generated in this study, have been submitted to the NCBI Gene Expression Omnibus (GEO; http://www.ncbi.nlm.nih.gov/geo/) under accession number GSE145609 and also with further instructions under: https://github.com/pasquelab/totipotency. In addition, published datasets were downloaded as provided by Deng et al., $2014^{41}$ (GSE45719), Posfai et al., 20174 (GSE84892), Mohammed et al., $2017^{42}$ (GSE100597), Chen et al., 2016 ${ }^{43}$ (GSE74155), Li et 
al., 201939 (GSE135289, GSE135701), Yang et al., $2017^{35}$ (ERP005641), Yang et al., 2017 ${ }^{36}$

$\begin{array}{lllll}\text { (GSE89303) } \quad \text { Pijuan-Sala } & \text { al., } & 2019^{49}\end{array}$

(https://github.com/MarioniLab/EmbryoTimecourse2018), Janiszewski et al., $2019^{77}$

(GSE126229), Sozen et al., 20190 (GSE134240).

All other data supporting the findings of this study are available from the corresponding authors on reasonable request.

\section{References cited in Methods and Extended Data}

70 Blij, S., Parenti, A., Tabatabai-Yazdi, N. \& Ralston, A. Cdx2 efficiently induces trophoblast stem-like cells in naive, but not primed, pluripotent stem cells. Stem Cells Dev 24, 1352-1365, doi:10.1089/scd.2014.0395 (2015).

71 Hackett, J. A. et al. Tracing the transitions from pluripotency to germ cell fate with CRISPR screening. Nat Commun 9, 4292, doi:10.1038/s41467-018-06230-0 (2018).

72 Hadjantonakis, A. K. \& Papaioannou, V. E. Dynamic in vivo imaging and cell tracking using a histone fluorescent protein fusion in mice. BMC Biotechnol 4, 33, doi:10.1186/1472-6750-4-33 (2004).

73 Nagy, A., Rossant, J., Nagy, R., Abramow-Newerly, W. \& Roder, J. C. Derivation of completely cell culture-derived mice from early-passage embryonic stem cells. Proc Natl Acad Sci U S A 90, 8424-8428, doi:10.1073/pnas.90.18.8424 (1993).

74 Rugg-Gunn, P. J. et al. Cell-surface proteomics identifies lineage-specific markers of embryo-derived stem cells. Dev Cell 22, 887-901, doi:10.1016/j.devcel.2012.01.005 (2012).

75 Dobin, A. et al. STAR: ultrafast universal RNA-seq aligner. Bioinformatics 29, 15-21, doi:10.1093/bioinformatics/bts635 (2013).

76 Liao, Y., Smyth, G. K. \& Shi, W. The R package Rsubread is easier, faster, cheaper and better for alignment and quantification of RNA sequencing reads. Nucleic Acids Res 47, e47, doi:10.1093/nar/gkz114 (2019).

77 Janiszewski, A. et al. Dynamic reversal of random X-Chromosome inactivation during iPSC reprogramming. Genome Res 29, 1659-1672, doi:10.1101/gr.249706.119 (2019).

78 Love, M. I., Huber, W. \& Anders, S. Moderated estimation of fold change and dispersion for RNA-seq data with DESeq2. Genome Biol 15, 550, doi:10.1186/s13059014-0550-8 (2014).

79 Petropoulos, S. et al. Single-Cell RNA-Seq Reveals Lineage and X Chromosome Dynamics in Human Preimplantation Embryos. Cell 167, 285, doi:10.1016/j.cell.2016.08.009 (2016).

80 Picelli, S. et al. Tn5 transposase and tagmentation procedures for massively scaled sequencing projects. Genome Res 24, 2033-2040, doi:10.1101/gr.177881.114 (2014).

81 Ramskold, D., Wang, E. T., Burge, C. B. \& Sandberg, R. An abundance of ubiquitously expressed genes revealed by tissue transcriptome sequence data. PLoS Comput Biol 5, e1000598, doi:10.1371/journal.pcbi.1000598 (2009). 
82 Butler, A., Hoffman, P., Smibert, P., Papalexi, E. \& Satija, R. Integrating single-cell transcriptomic data across different conditions, technologies, and species. Nat Biotechnol 36, 411-420, doi:10.1038/nbt.4096 (2018).

83 Huynh-Thu, V. A., Irrthum, A., Wehenkel, L. \& Geurts, P. Inferring regulatory networks from expression data using tree-based methods. PLoS One 5, doi:10.1371/journal.pone.0012776 (2010).

84 Moerman, T. et al. GRNBoost2 and Arboreto: efficient and scalable inference of gene regulatory networks. Bioinformatics 35, 2159-2161, doi:10.1093/bioinformatics/bty916 (2019).

85 Vintersten, K. et al. Mouse in red: red fluorescent protein expression in mouse ES cells, embryos, and adult animals. Genesis 40, 241-246, doi:10.1002/gene.20095 (2004).

86 Gertsenstein, M. Mouse embryos' fusion for the tetraploid complementation assay. Methods Mol Biol 1313, 41-59, doi:10.1007/978-1-4939-2703-6_3 (2015). 

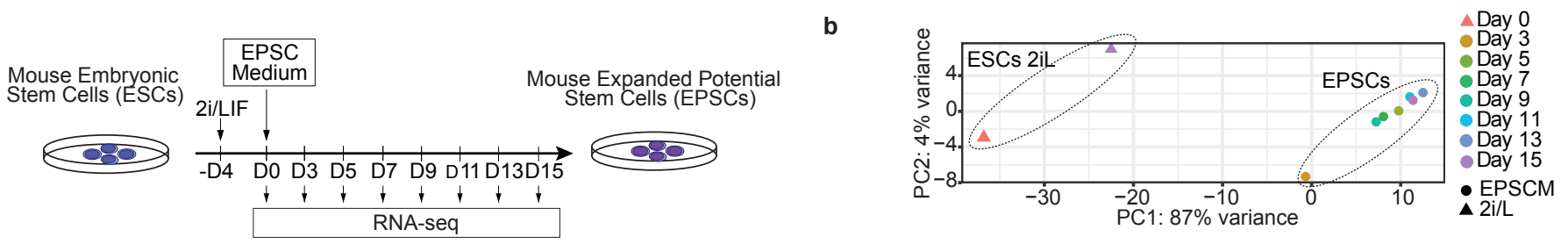
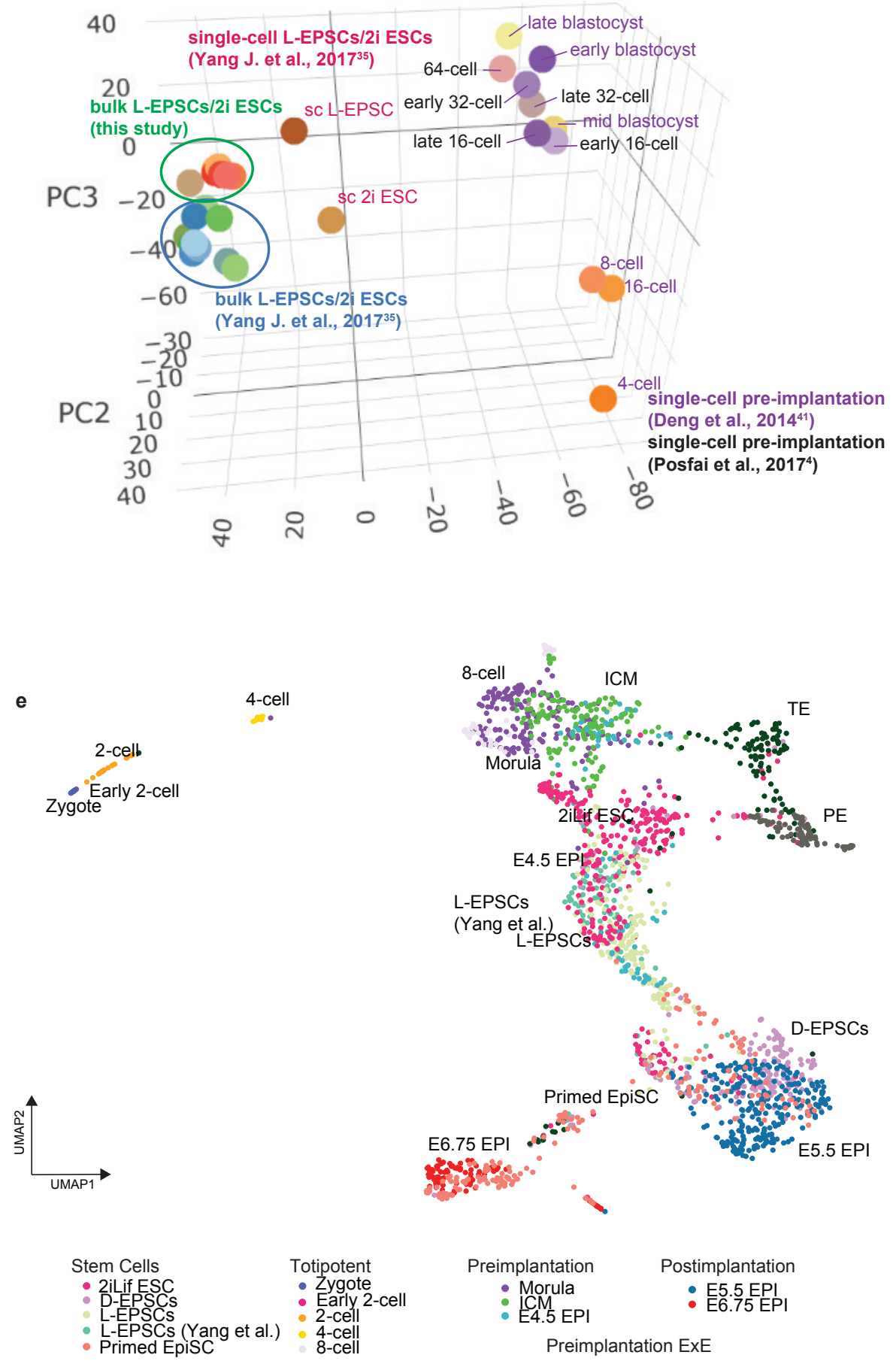

$\begin{array}{cc}\text { Preimplantation } & \text { Postimplantation } \\ \text { Morula } & \text { E5.5 EPI }\end{array}$

: MCM : E5.5 EPI

Preimplantation ExE d

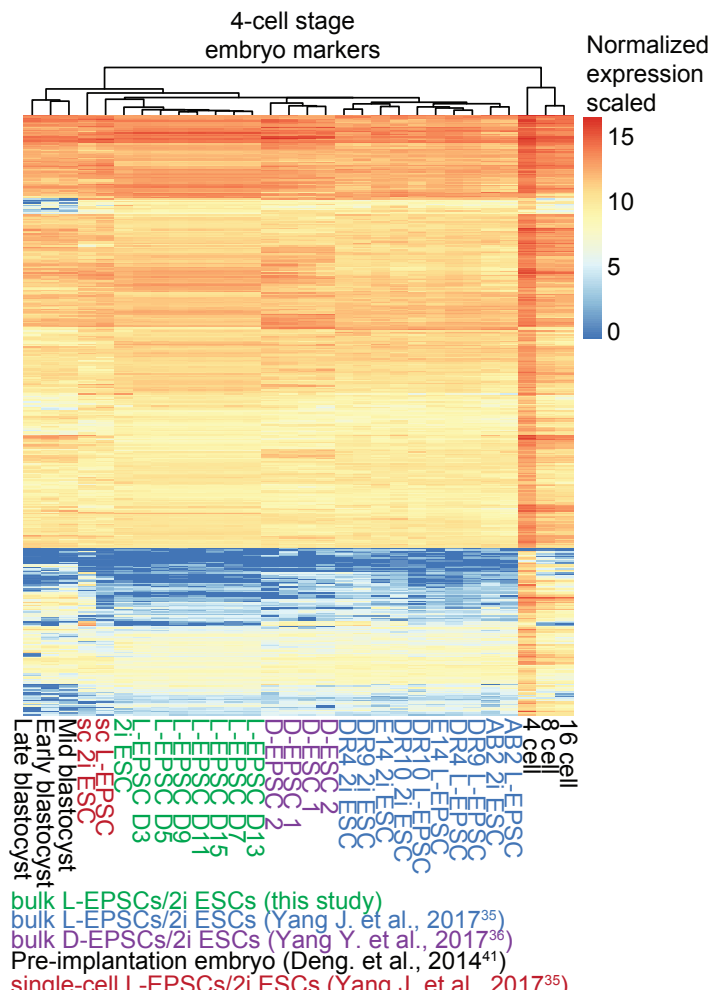

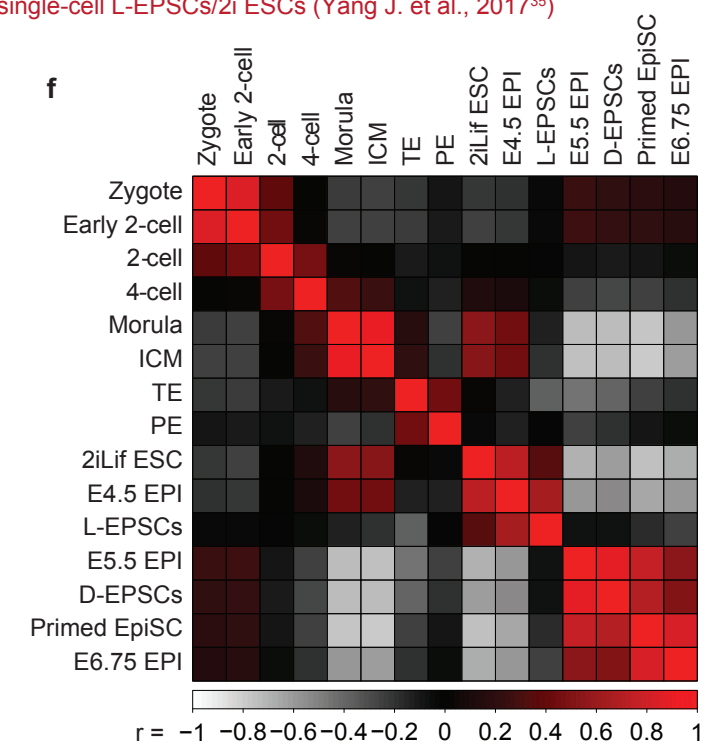

- $\mathrm{TE}$ 
a

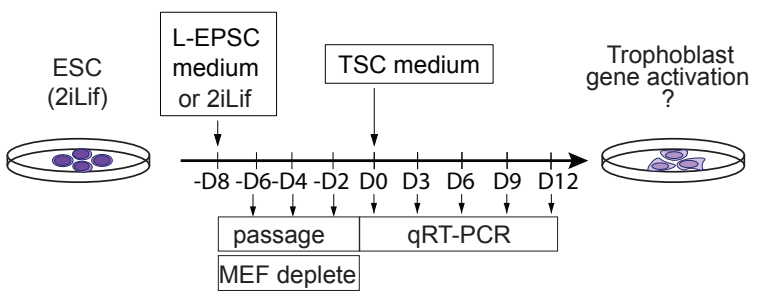

b

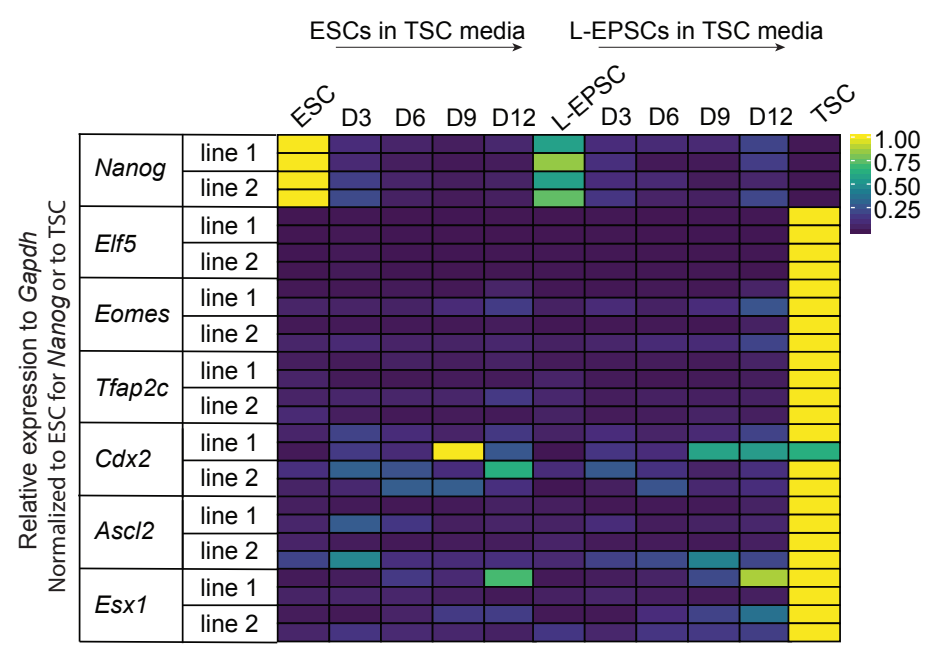

c

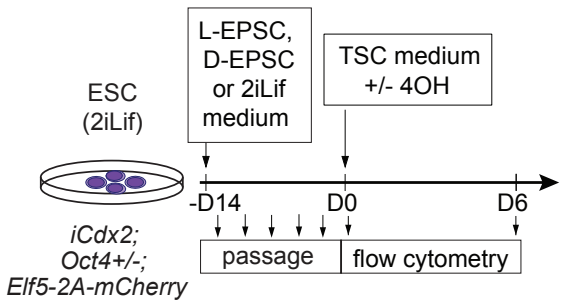

CD40/Elf5/Plet1 activation

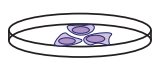

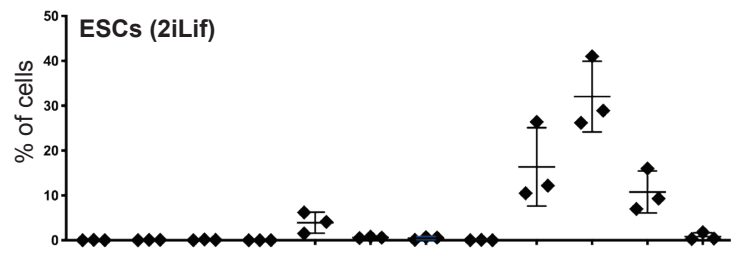
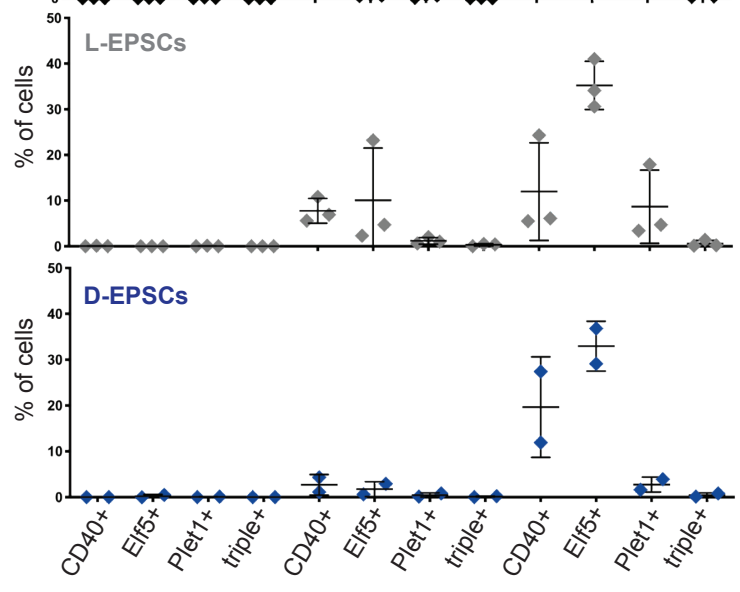

day 0

day $6-4 \mathrm{OH}$

day $6+4 \mathrm{OH}$

TSCs

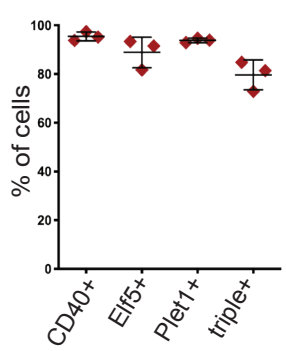


L-EPSCs (Yang et al., 201735)

L-EPSCs

- D-EPSCs

- EpisCs

Blastoids

B-Blastoid-EPI

- B-Blastoid-PE

- B-Blastoid-TE

B-Blastoid-Int-1

- B-Blastoid-Int-2

- ZG-Blastoid-EPI

ZG-Blastoid-Int-

ZG-Blastoid-PE

- ZG-Blastoid-TE
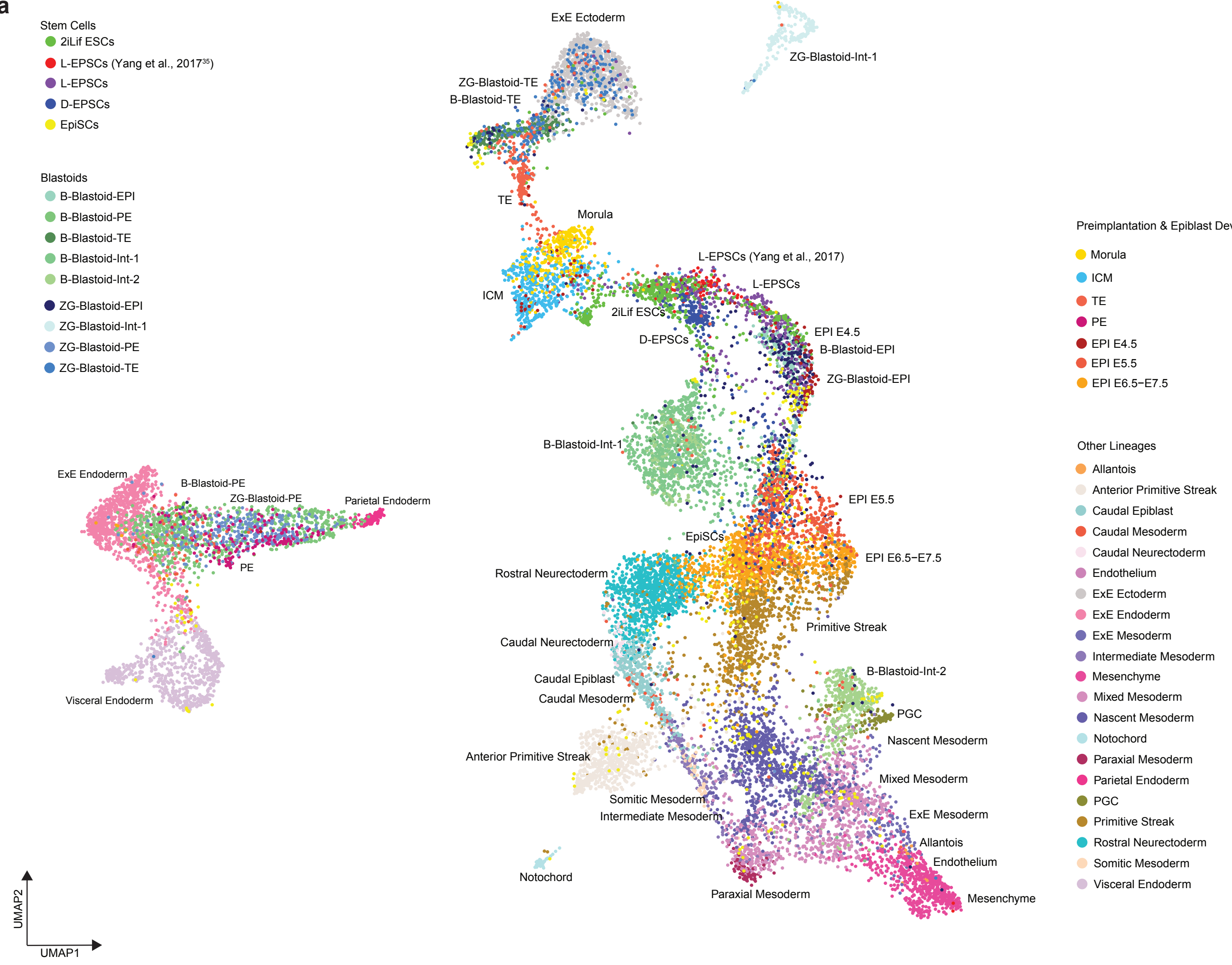

C

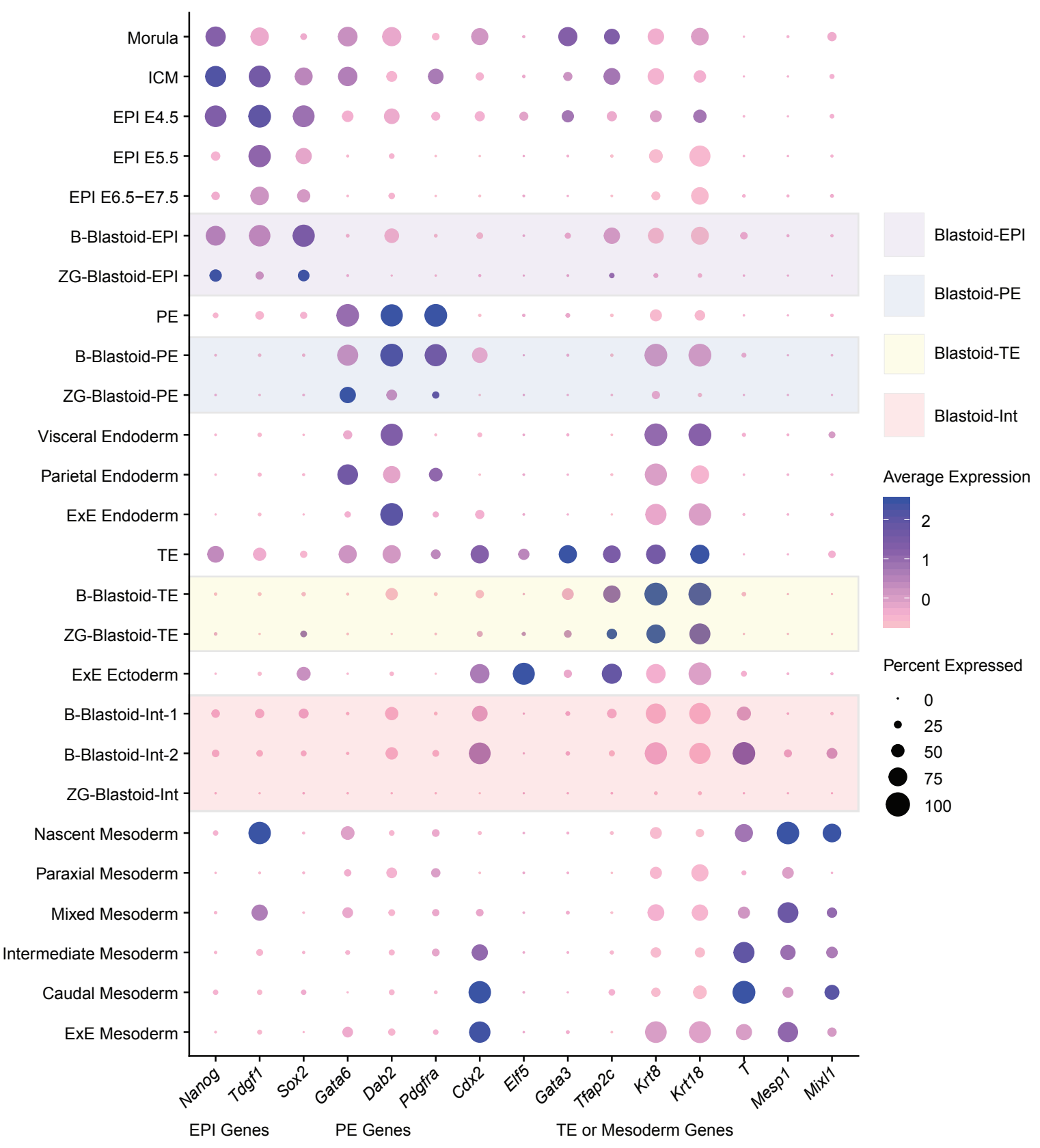

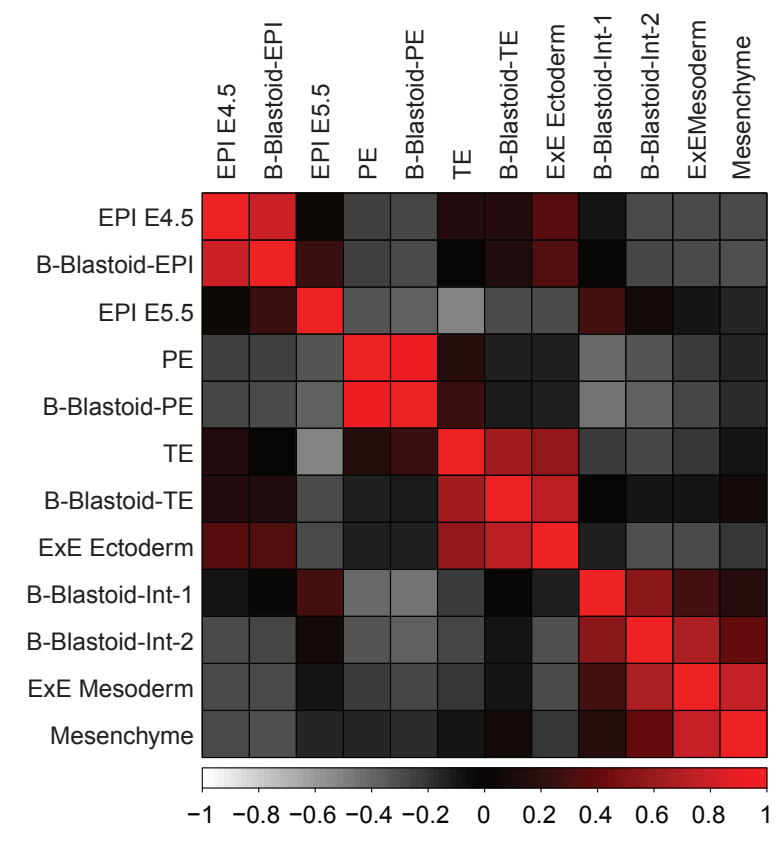

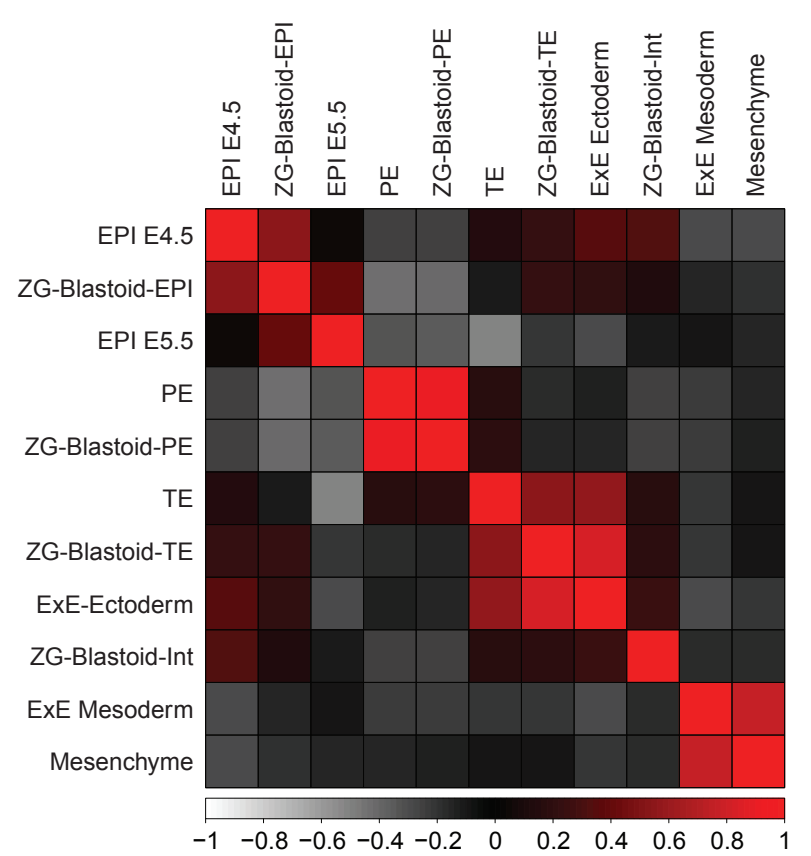


a

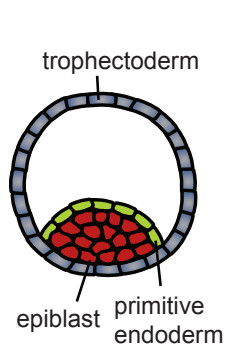

d

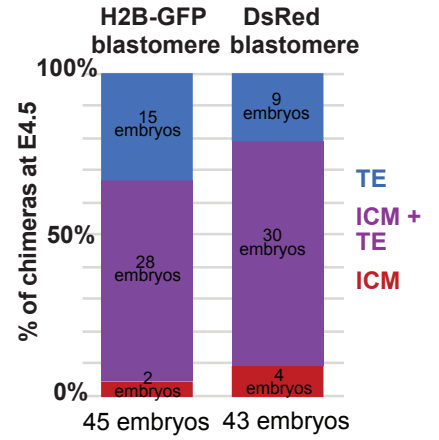

E4.5 chimeras with different H2B-GFP stem cells

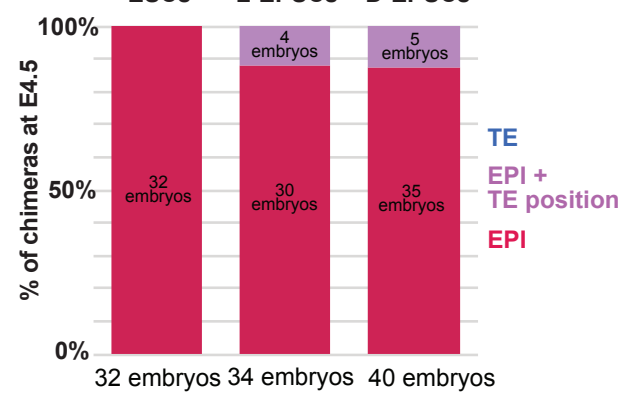
ESCs L-EPSCs D-EPSCs

e E4.5 chimeras with H2B-Gfp D-EPSCs

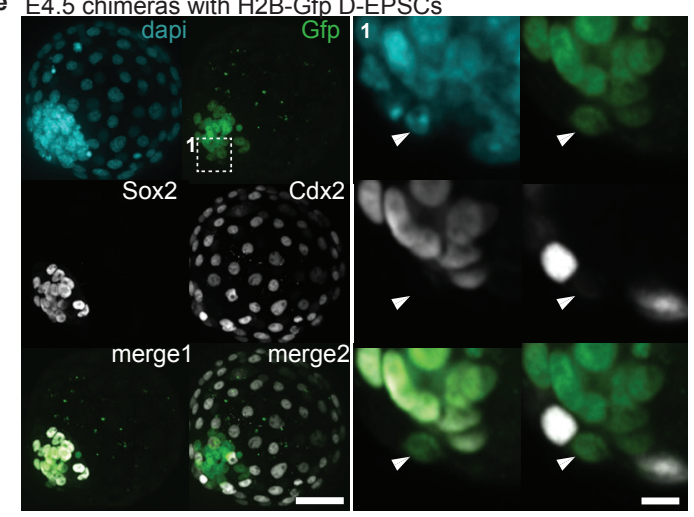

E4.5 chimeras with totipotent H2B-Gfp blastomeres
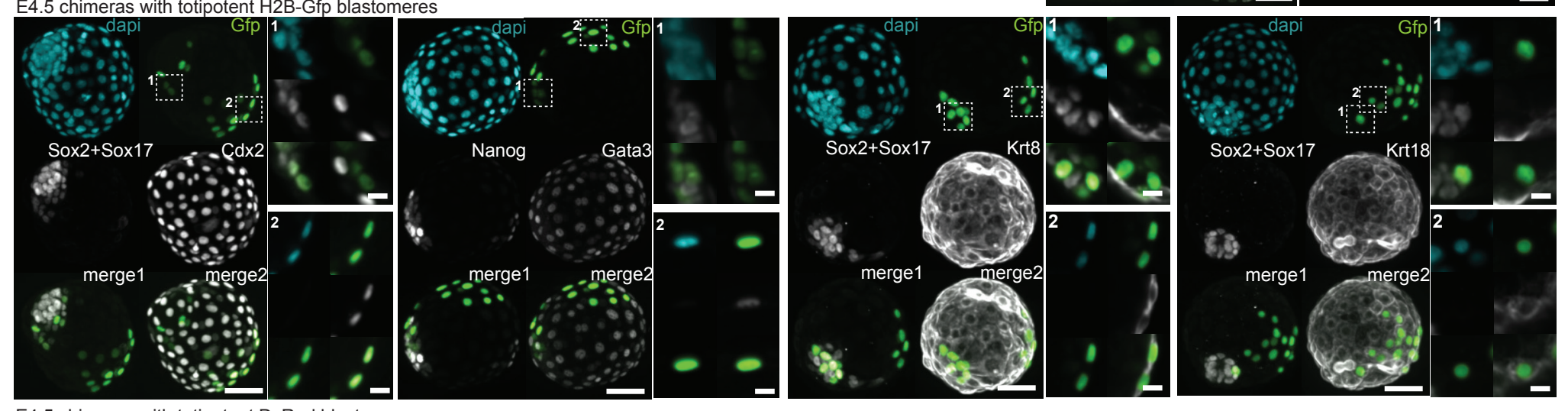

E4.5 chimeras with totipotent DsRed blastomeres
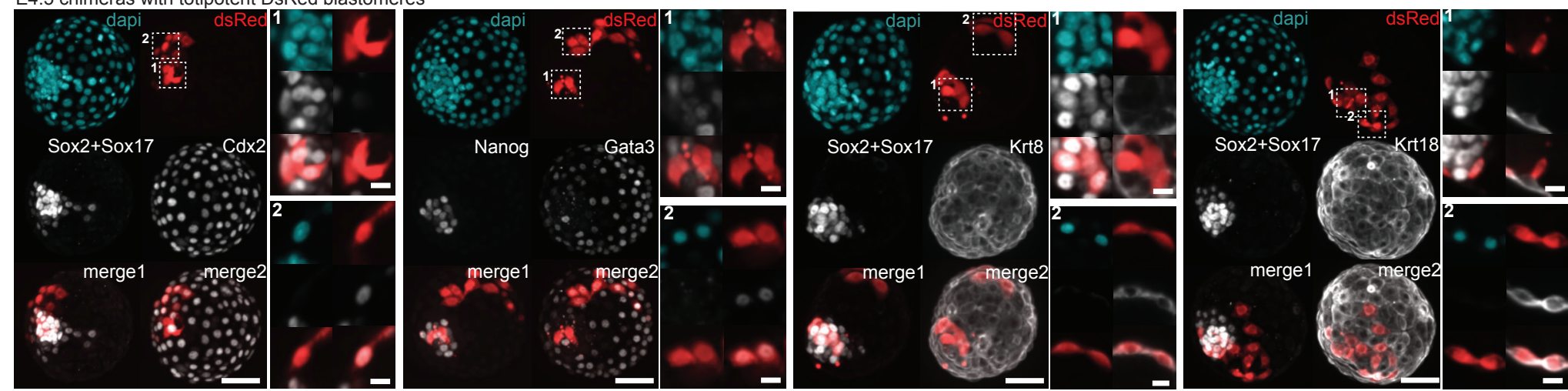
b E6.25 chimeras with totipotent H2B-Gfp blastomeres
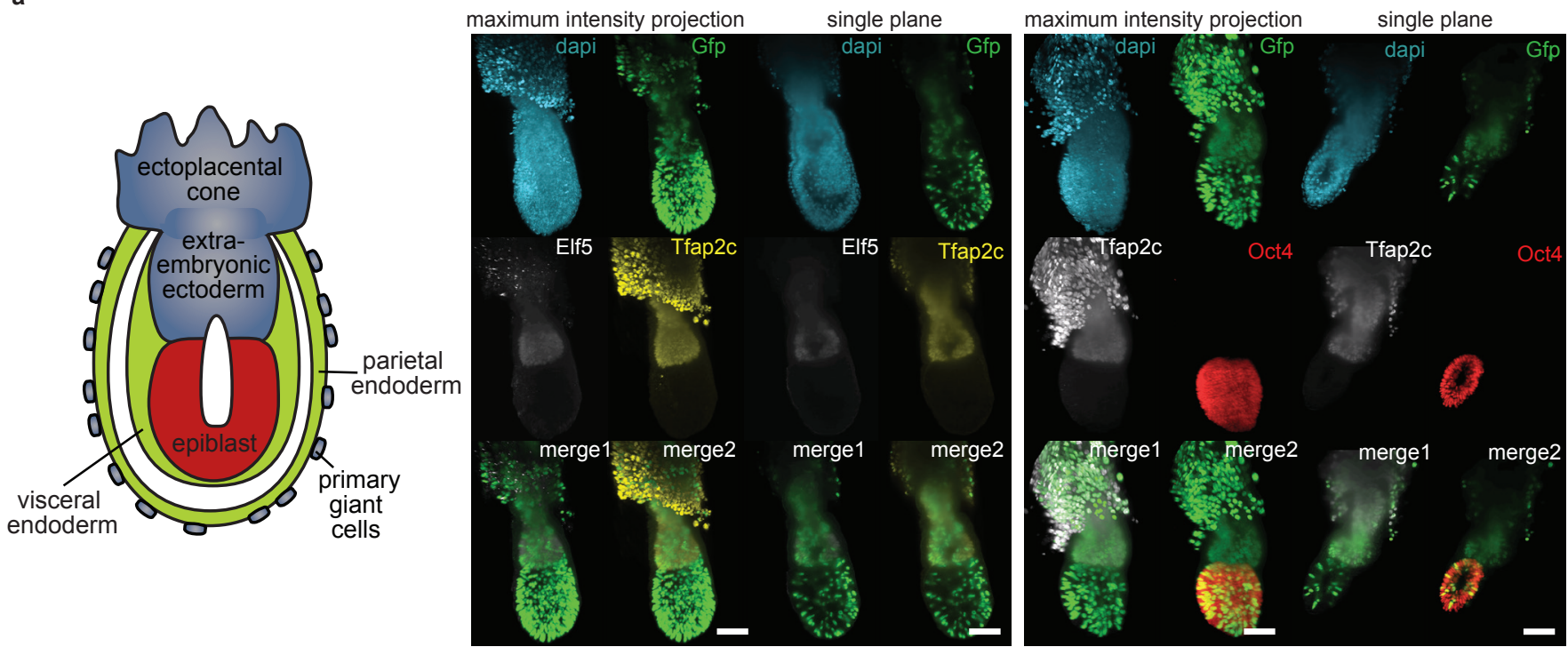

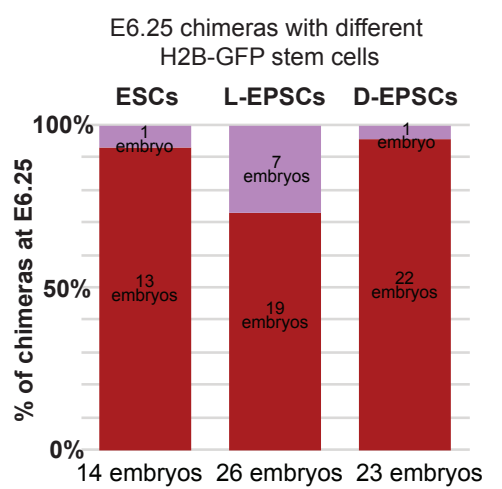

EPI +

TB position

EPI d E6.25 chimeras with H2B-Gfp L-EPSCs

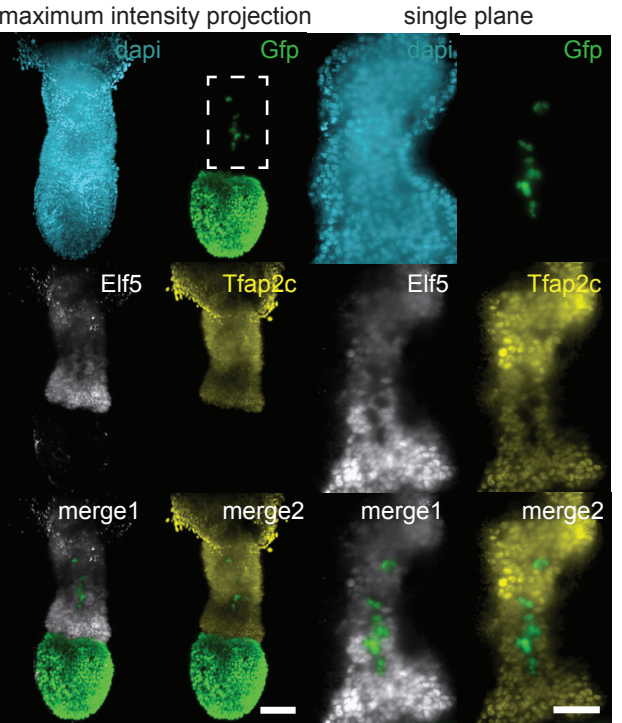

maximum intensity projection single plane

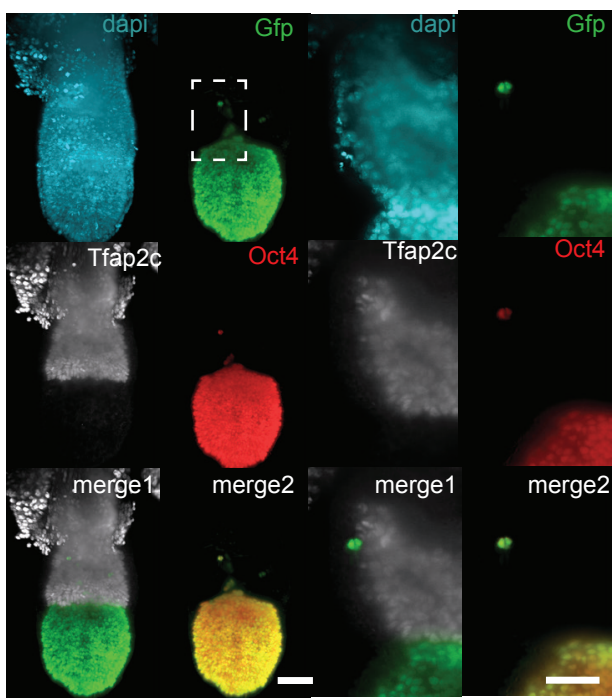


a

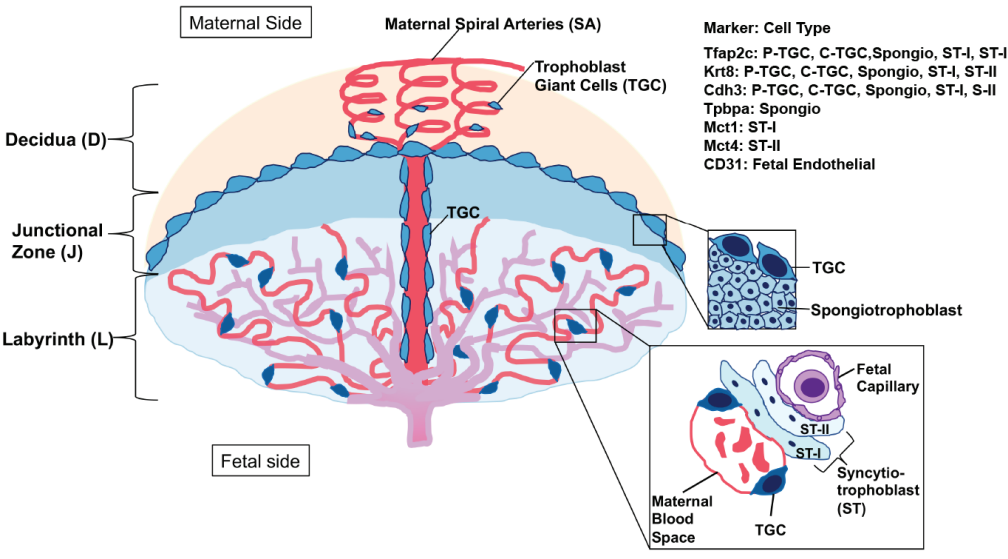

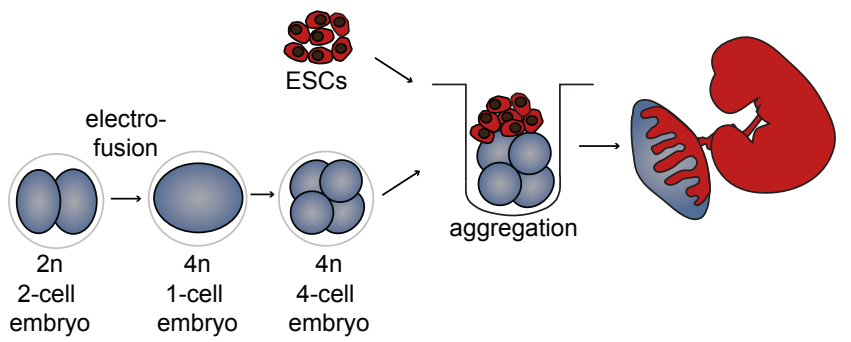

H2B-Gfp tetraploid host embryo + wild-type ESCs

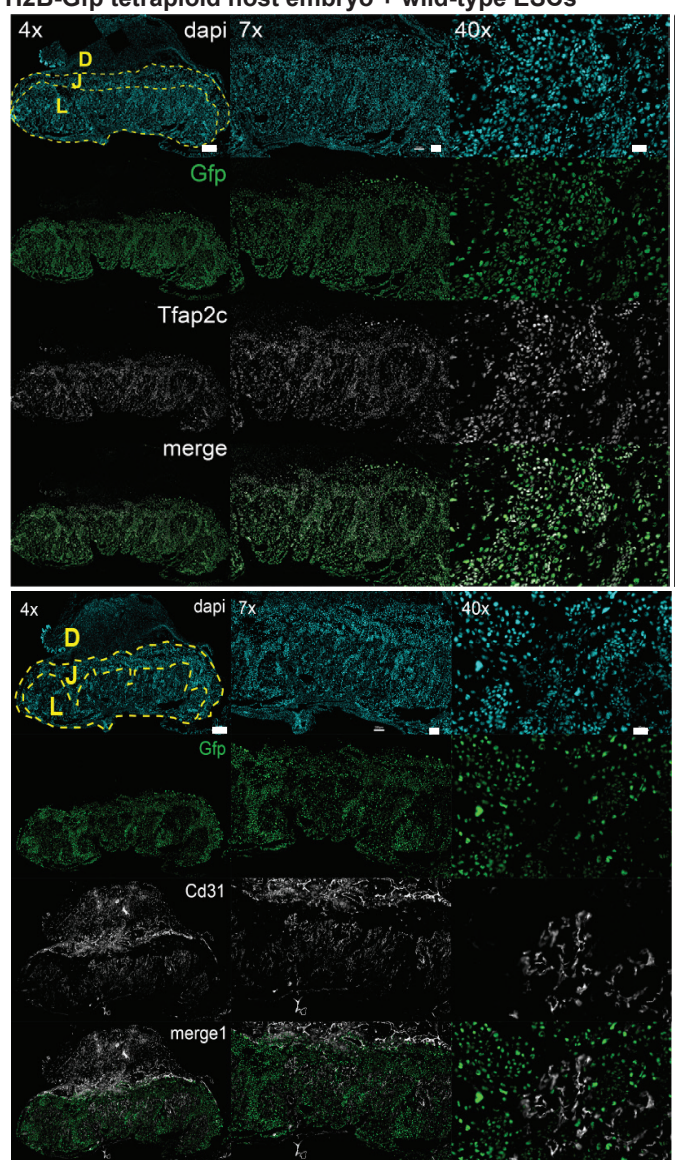

wild-type tetraploid host embryo + H2B-Gfp ESCs
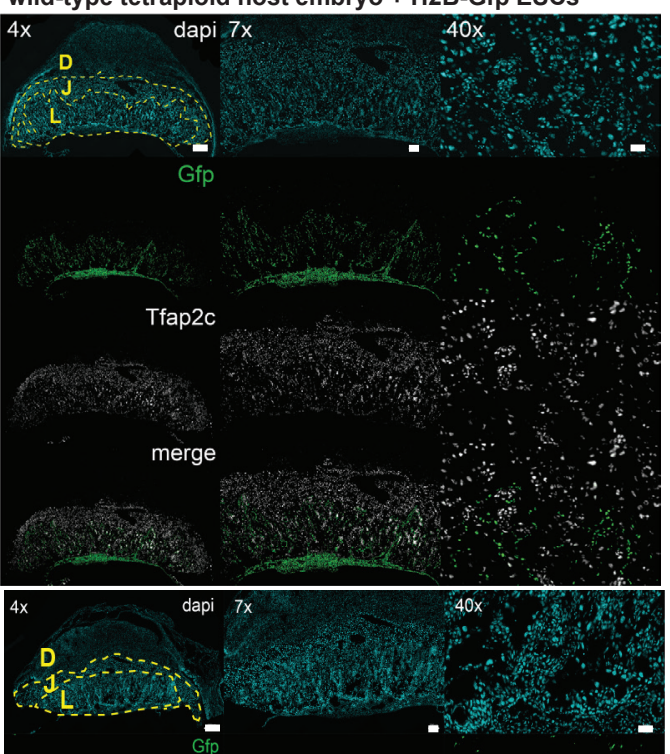

Cd3

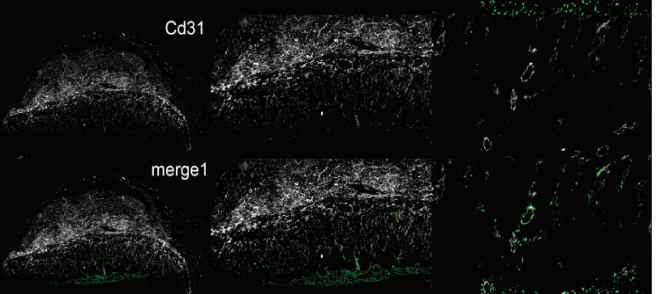

wild-type diploid host embryo + H2B-Gfp L-EPSCs

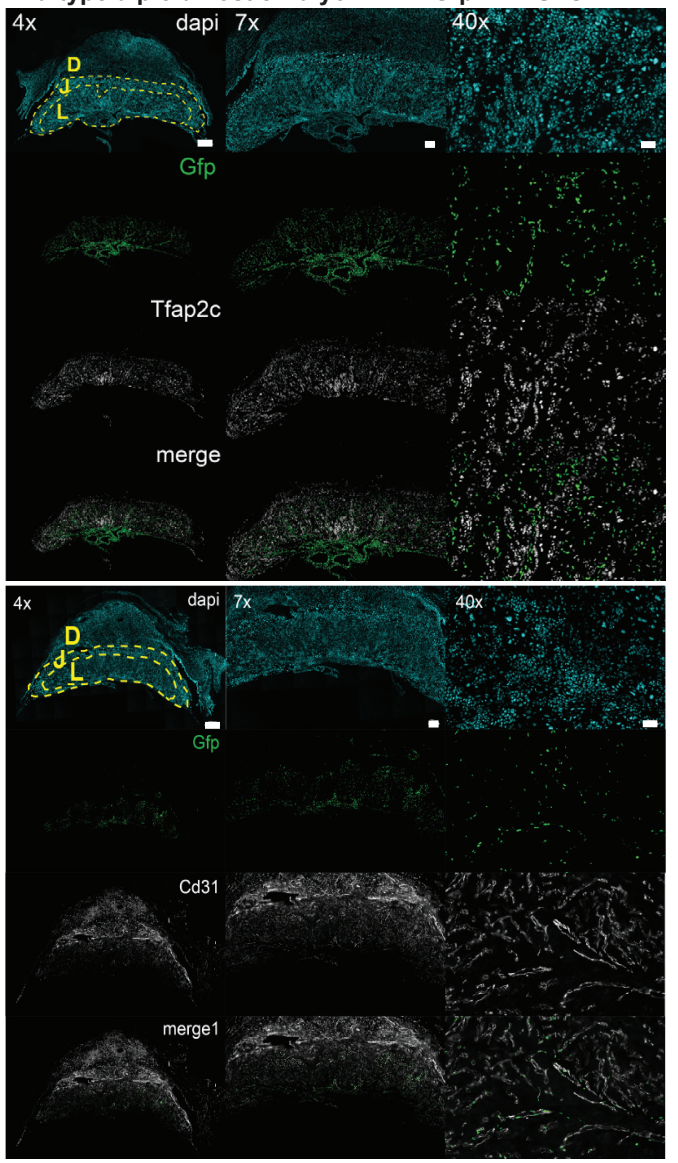




$\begin{array}{ll}\text { D-EPSC } & \text {, } \\ \text { hLIF } & \text { Ssk3 inhibitor (CHIR99021) } \\ \text { Dimethendine maleate } & \text { (anti-histamine) } \\ \text { Minocycline hydrochloride } & \text { (Parp1 inhibition) }\end{array}$

L-EPSC
hLIF
Mek inhitor (PD0325901)
Psk3 inhibitor (CHIR99021)
Jnk inhibtor (SB203580)
Src inhibitor (TCS JNK 60)
Wnt inhibitor (XAV939)
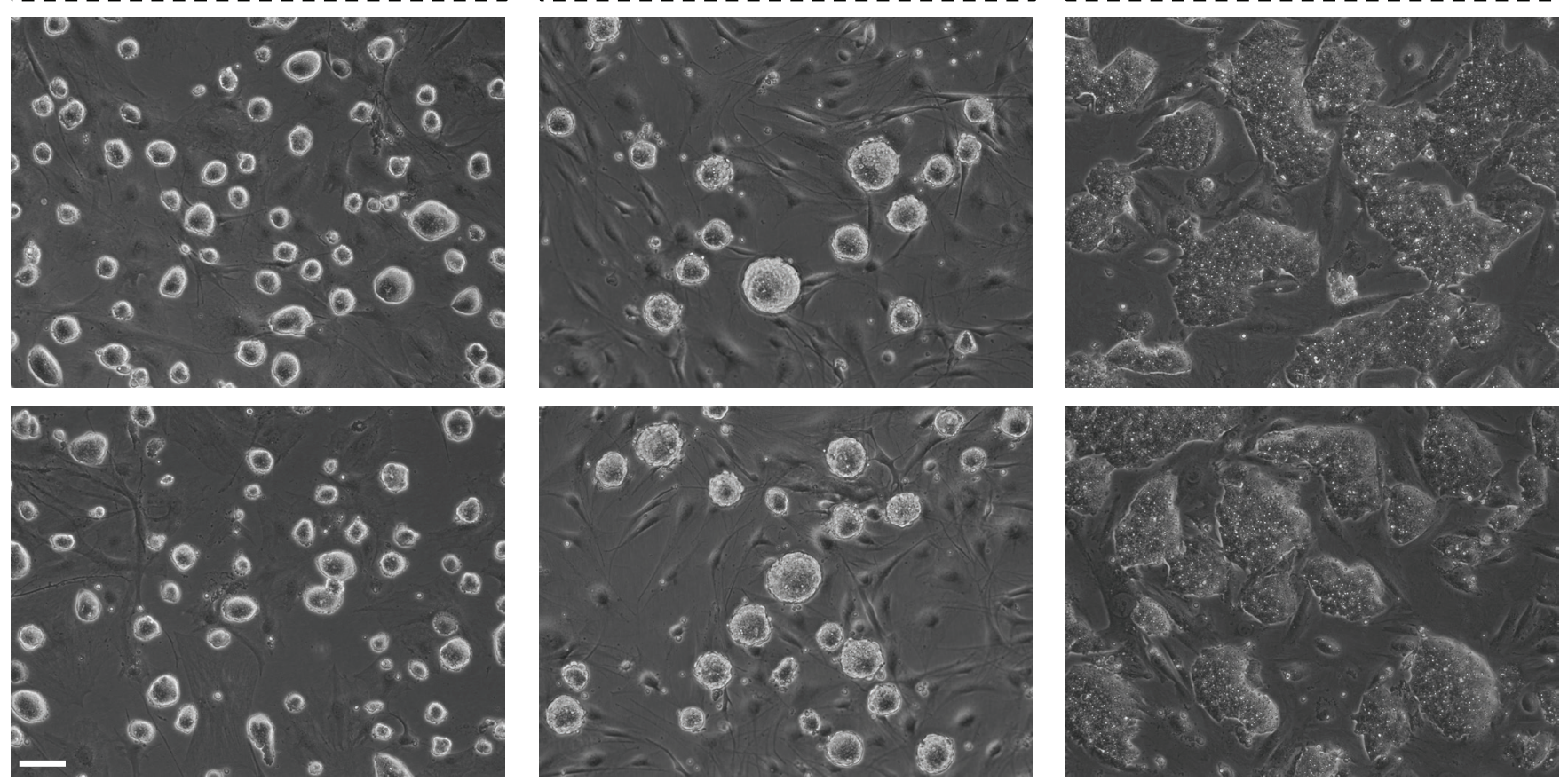


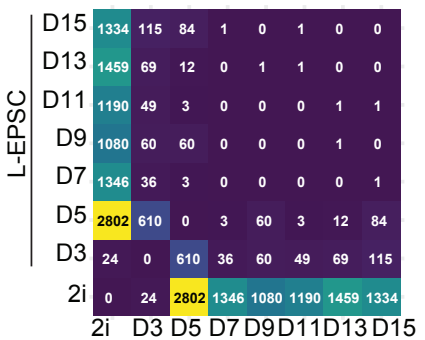
\# of DEGs $\frac{2 i}{\text { L-EPSC }}$ $v_{0} 0_{0} 0_{0} 0$ b
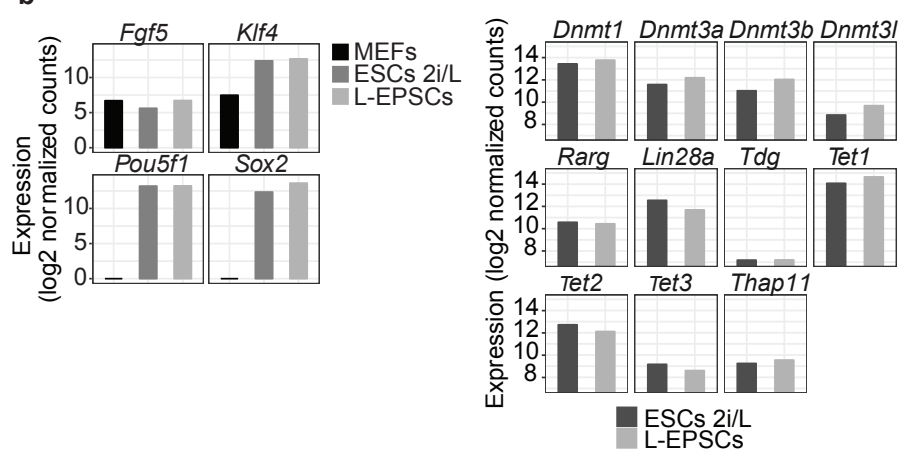

bulk L-EPSCs (Yang J. et al., 2017)

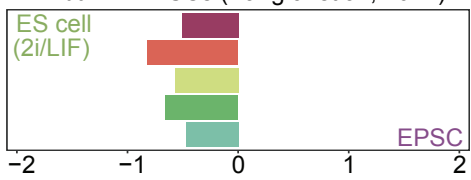

bulk L-EPSCs (this study)

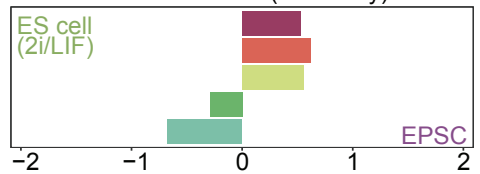

${ }^{-2}$ bulk $^{-1} \quad \stackrel{0}{0}$-EPSCs (Yang Y. et al., 2017)

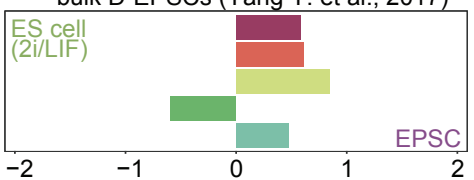

Normalized gene set enrichment score

- Zygote

Two-cell

Four-cell

Eight-cell

Sixteen-cell
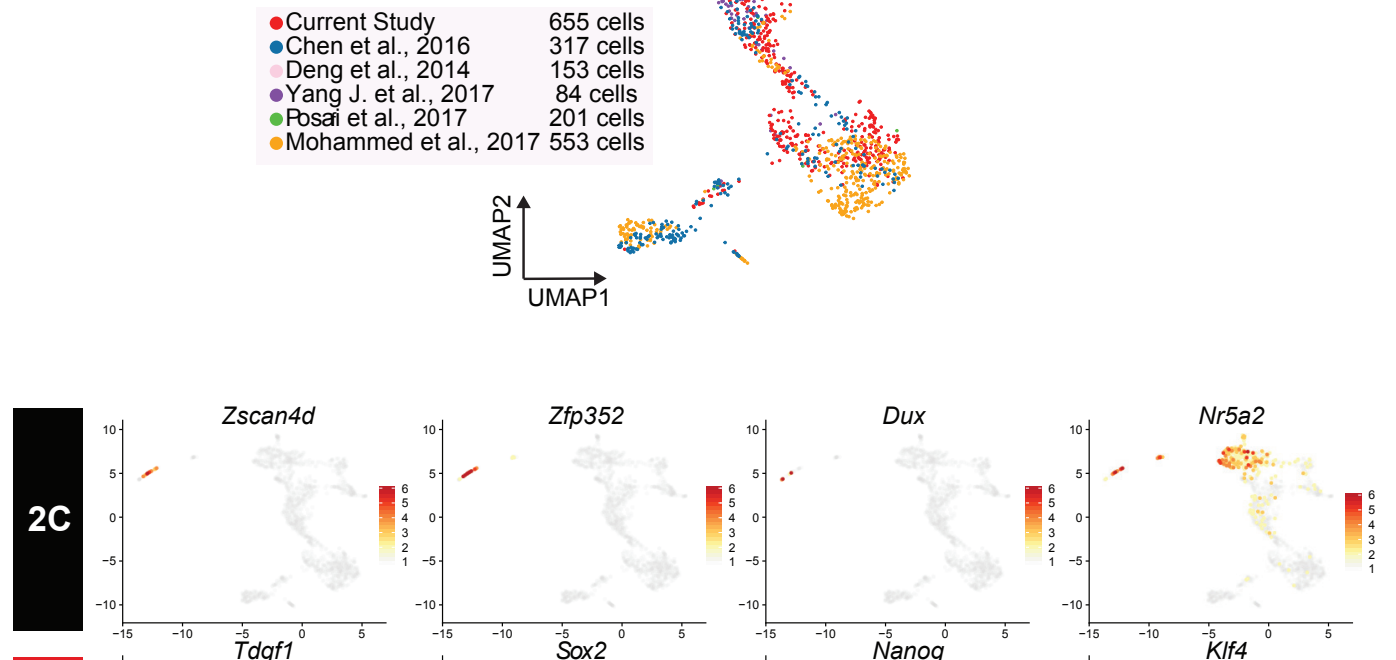

g

sc L-EPSCs (Yang J. et al., 2017)
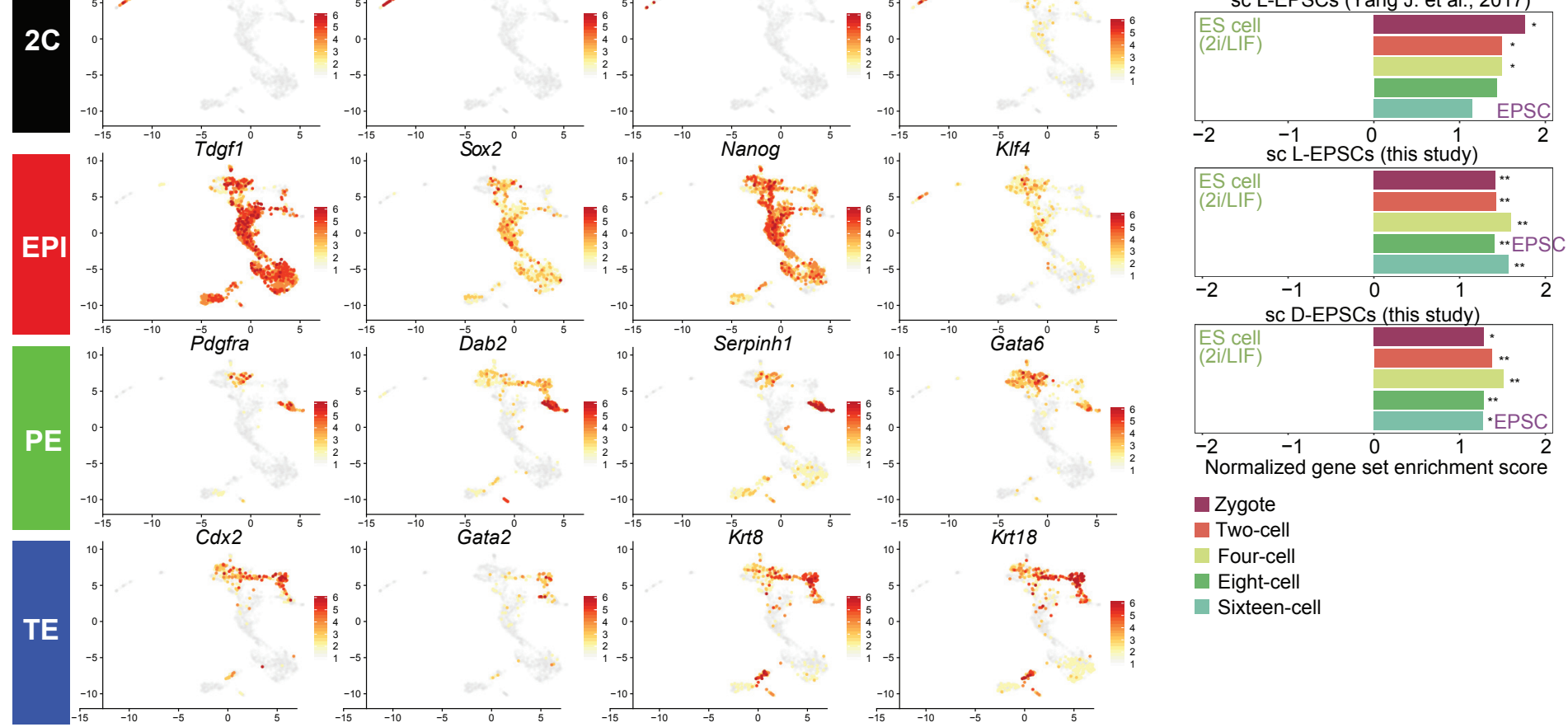

Normalized gene set enrichment score

Zygote

Two-cell

Four-cell

- Eight-cell

Sixteen-cell

h

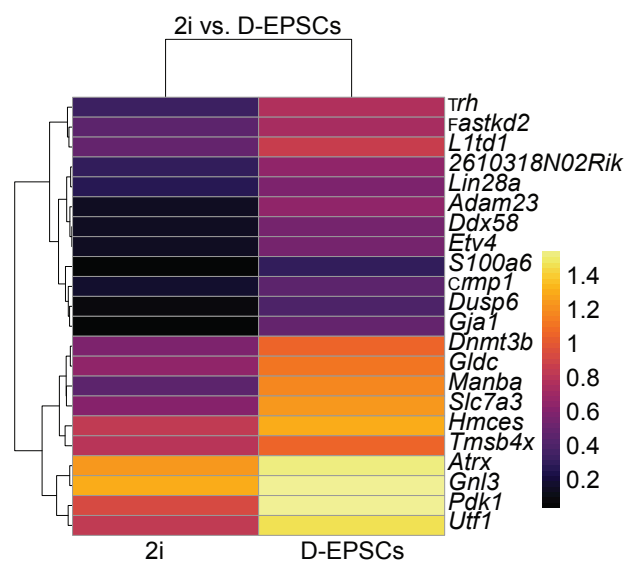

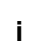

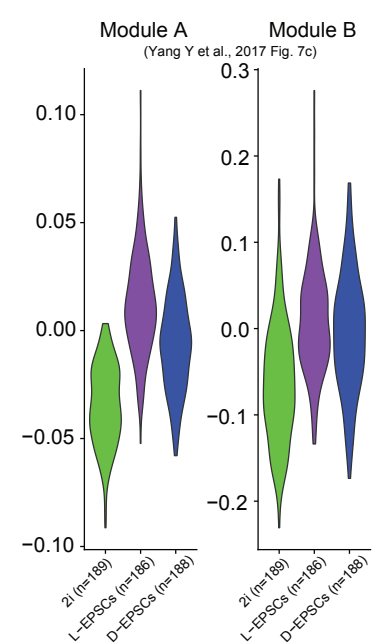




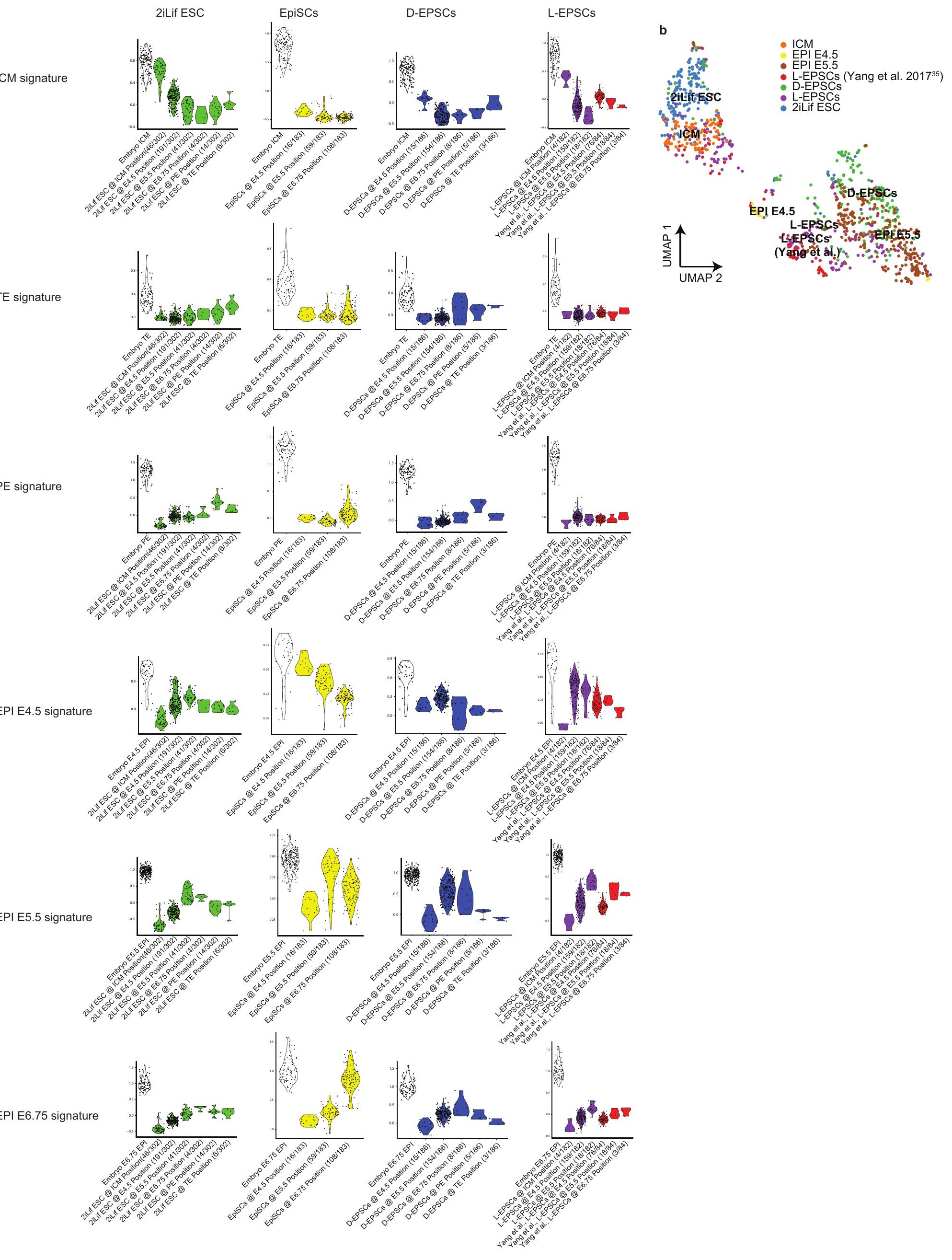



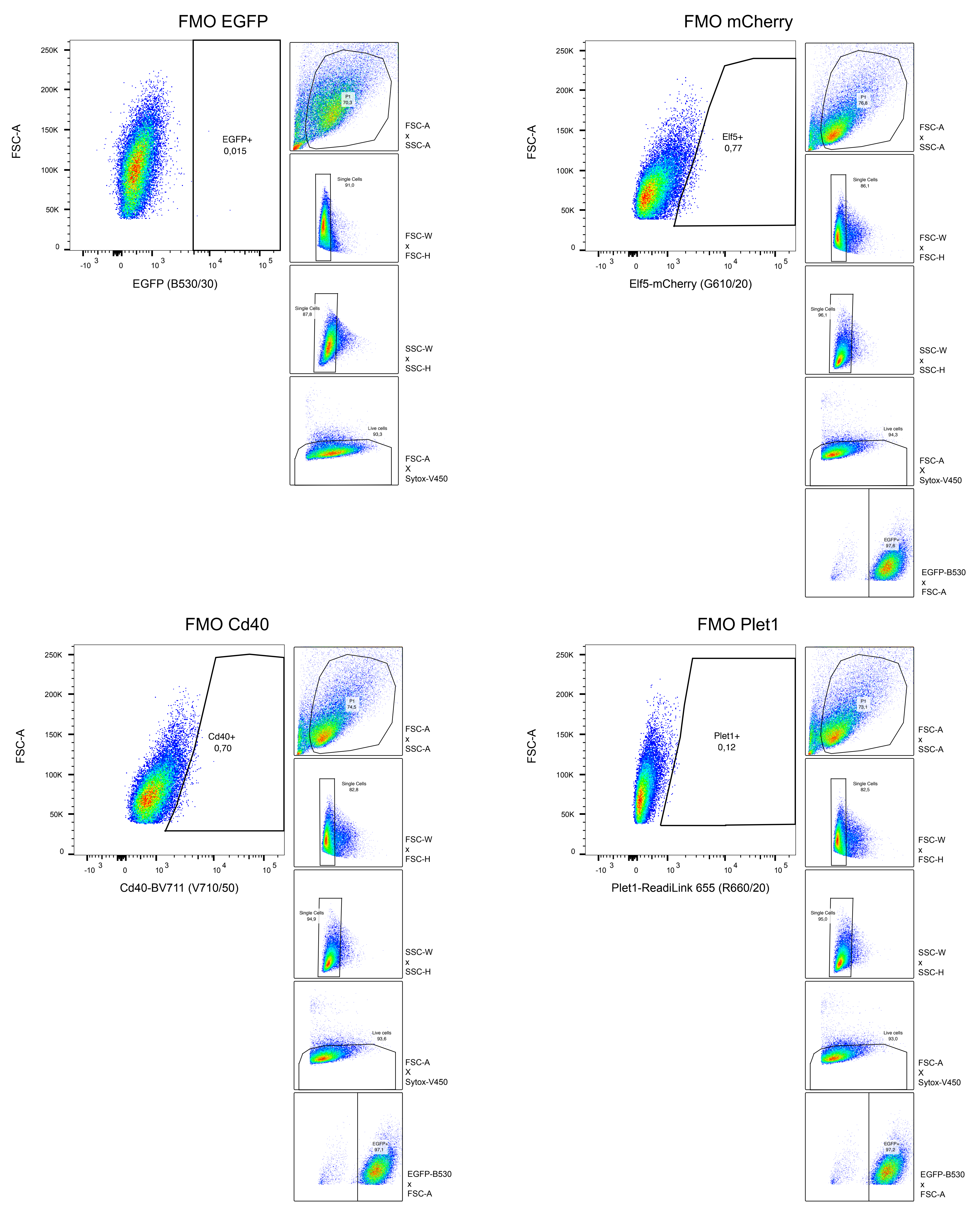

Flow cytometry gating strategy related to Figure 2 .

Displayed is an example of the gating strategy implemented to generate flow cytometry data. The data displayed uses the iCdx2 Elf5.2A.mCherry ESCs, which are eGFP+, contain the Elf5.2A.mCherry reporter, and are stained for both CD40 and Plet1. The plots are pseudo-colour plots and the $x$-axis labels state markers and fluorochromes used. Laser ( $V=$ violet; $B=$ blue; $G=$ green; $R=$ red) and filter bandpass information is also included on $x$-axis in brackets.

Ancestry plots are included to show how cells were gated: majority of cells were included in FSC/SSC gate due to size discrepancy between cells of interest and underlying feeder cells. Single cells were determined by FSC-W x FSC-H and SSC-W x SSC-H plots.

Live cells were gated using dead cell stain Sytox blue. Cells of interest were discriminated from underlying feeder cells (EGFP negative) by expression of eGFP.

Fluorescence minus one (FMO) controls were used to establish gates in order to separate positive and negative populations. 


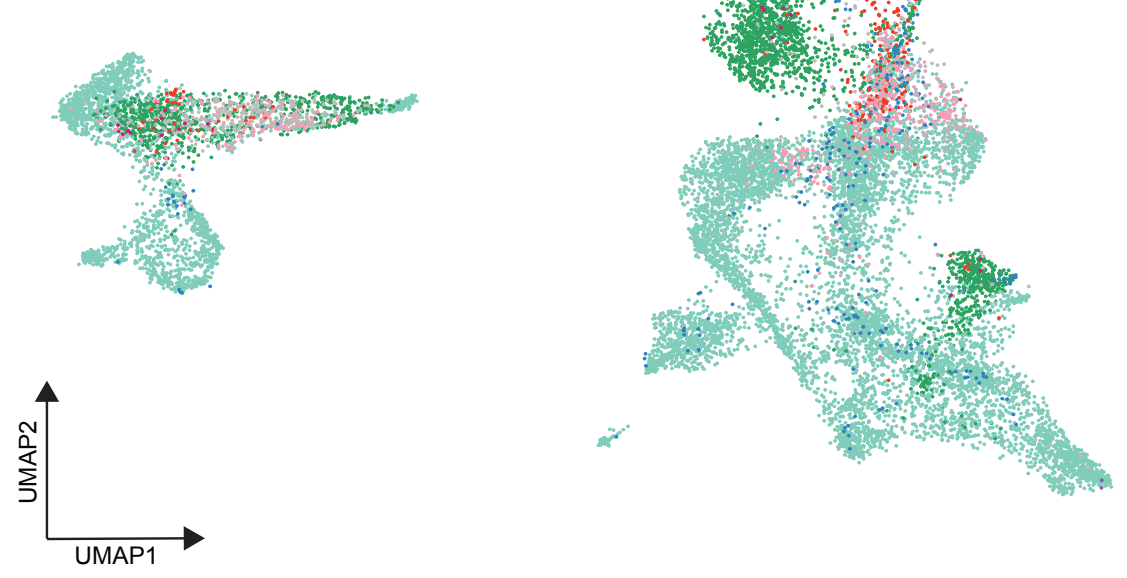

Current Study
Chen et al., 2016
Li et al., $2019^{39}$
Yang et al., $2017^{35}$
Mohammed et al., 201742
Pijuan-Sala et al., 201950
Posfai et al., $2017^{4}$
Sozen et al., $2019^{40}$

c

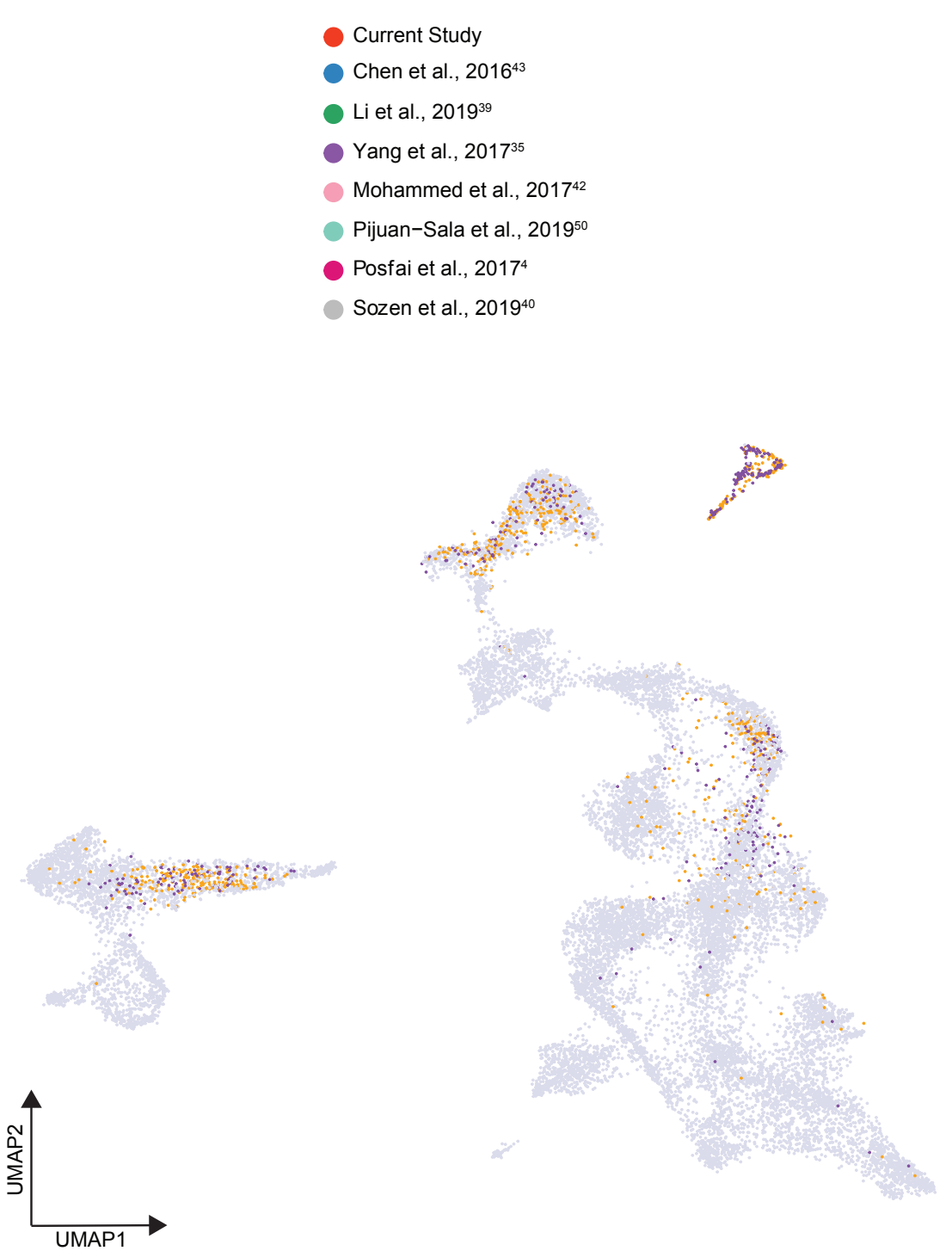

Sozen et al., $2019^{40}$ (ZG-Blastoids)

- 2iLif ZG-Blastoids

- EPSC ZG-Blastoids

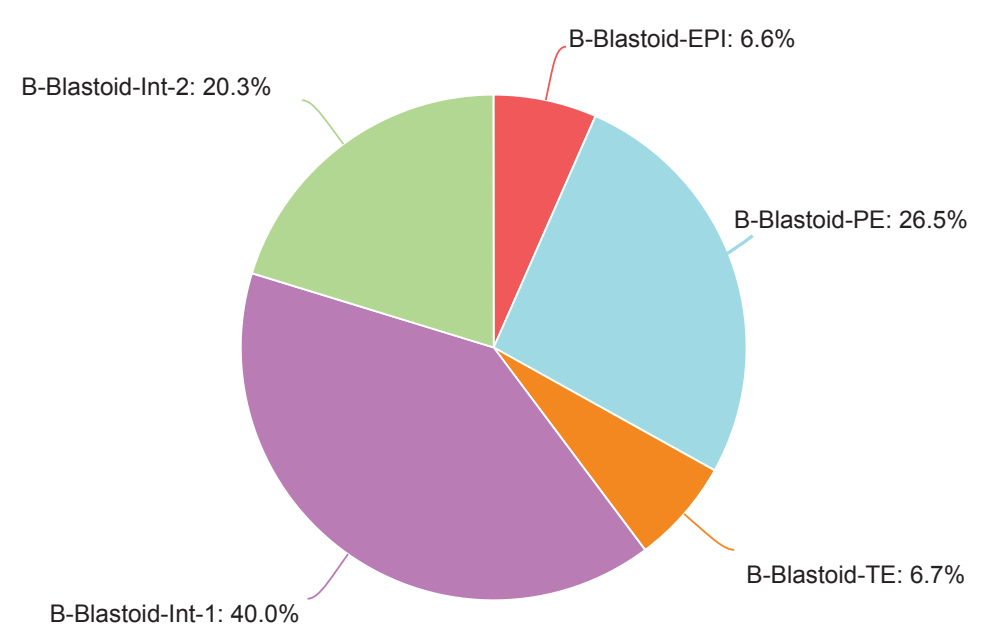

Li et al., 201939 (B-Blastoids)

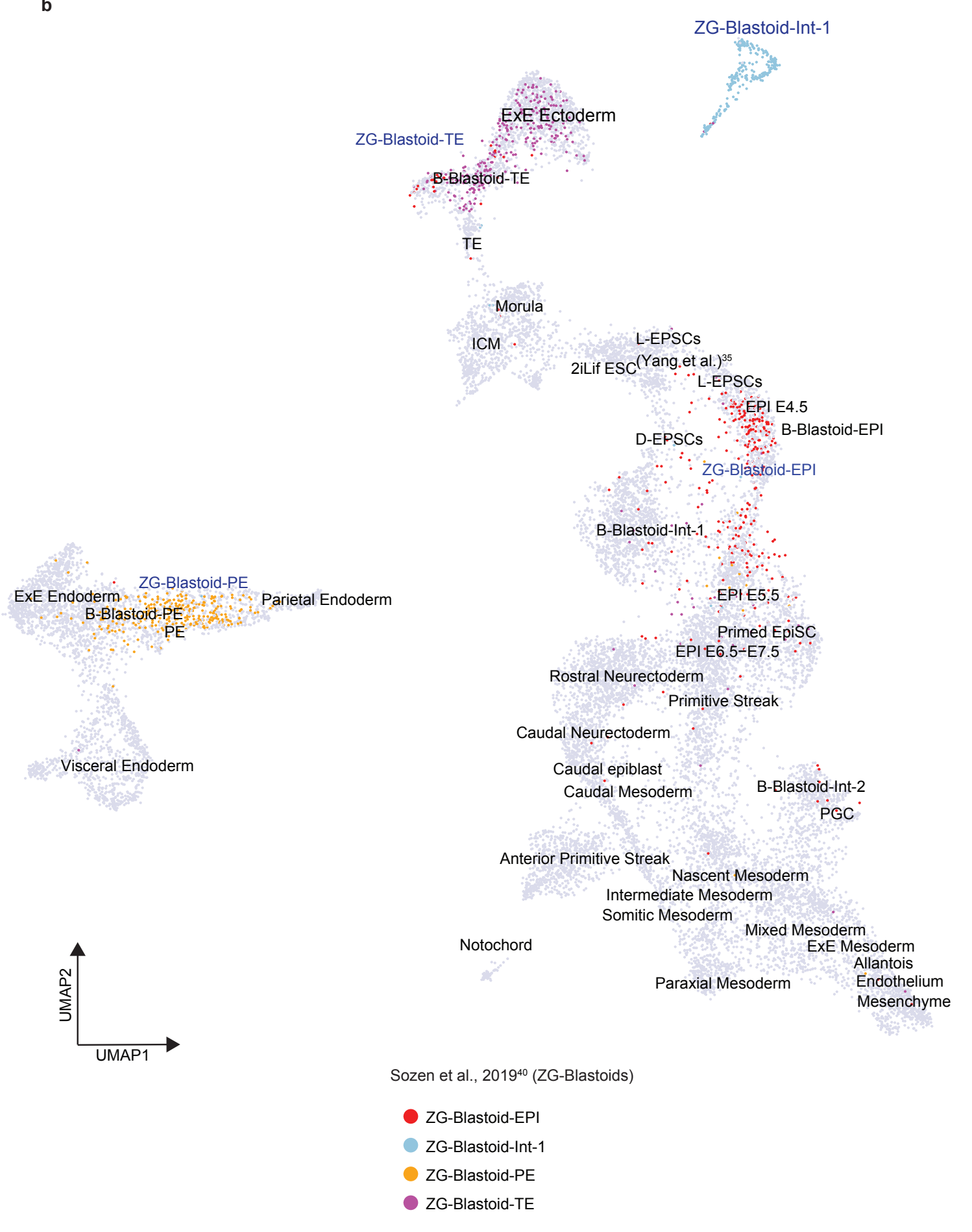

d
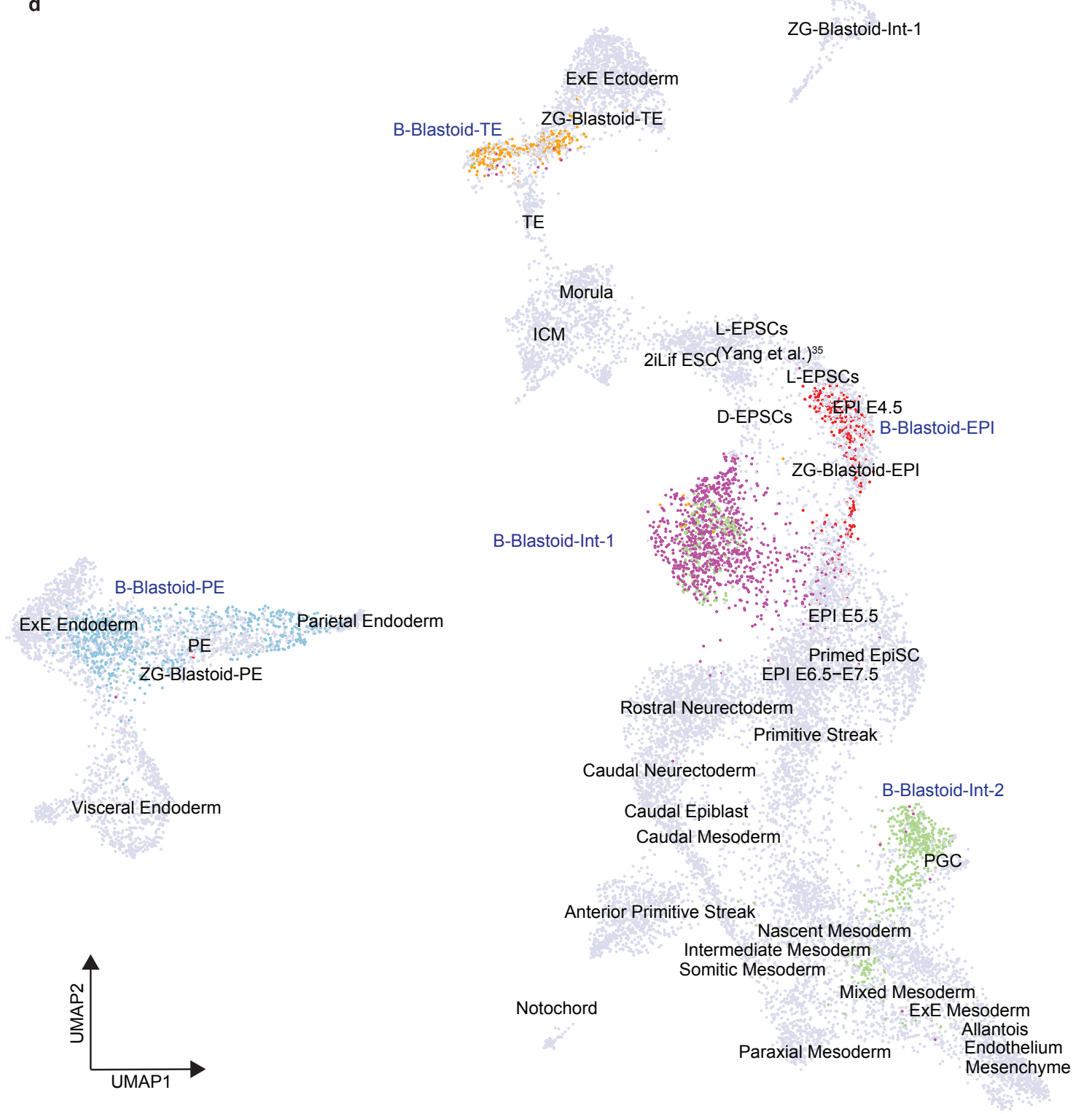
b
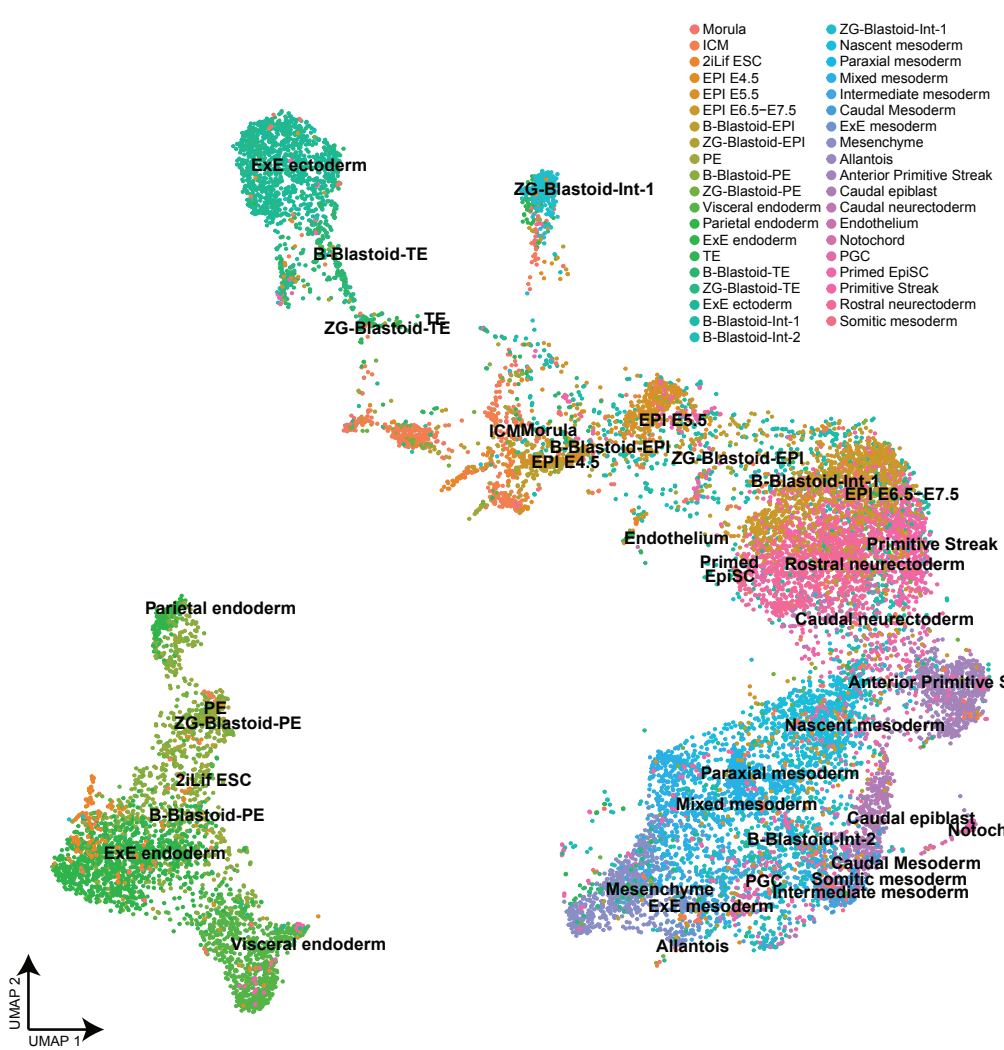

ZG-Blastoid-匯

B-Blastoid-Int-1

Rostral neurectoder
Somitic mesoderm

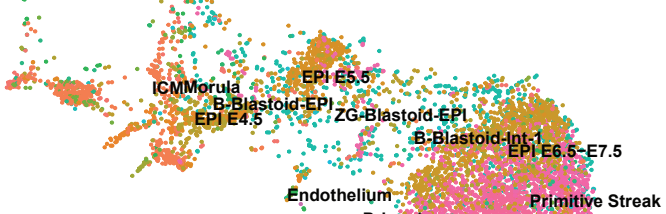
Episso Rostral neurectoderm Episcy
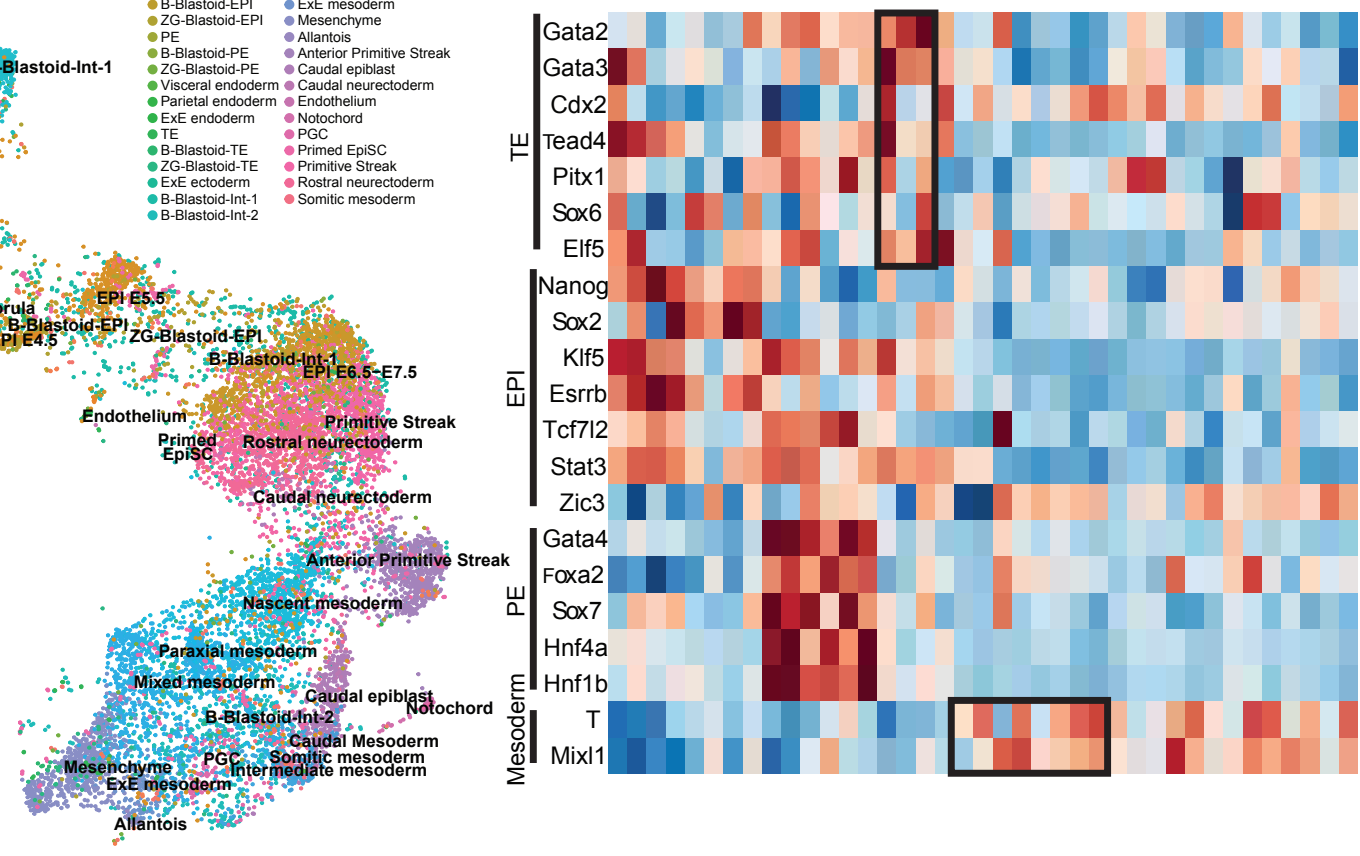

UMAP 1

c

Avg.
Expression

Nanog targets

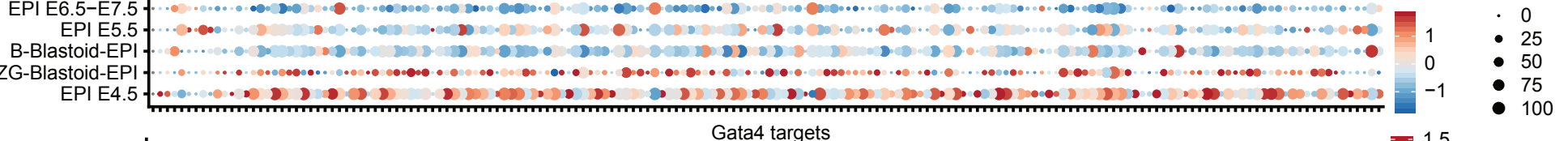

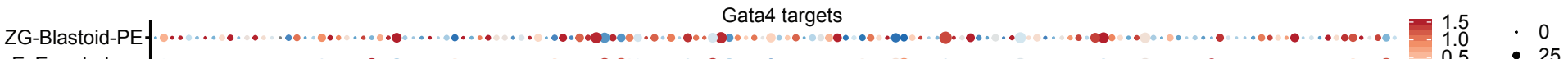

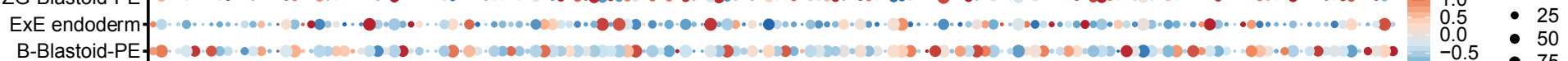

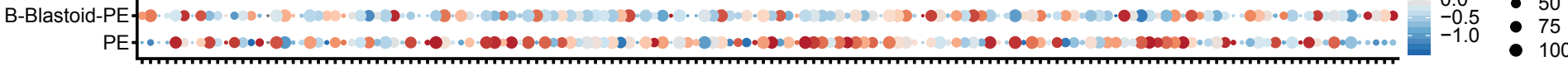

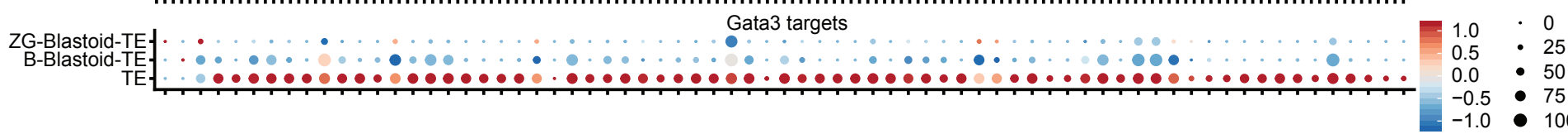

ZG-Blastoid-Int-1- . . . . . . . . T targets

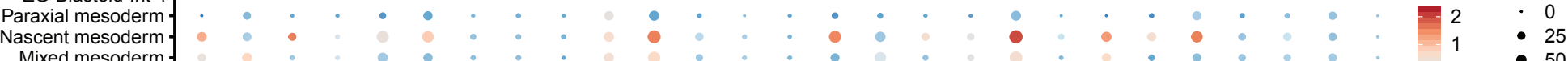
Intermediate mesoderm

B-Blastoid-Int-2

B-Blastoid-Int-1

Cdx2 targets

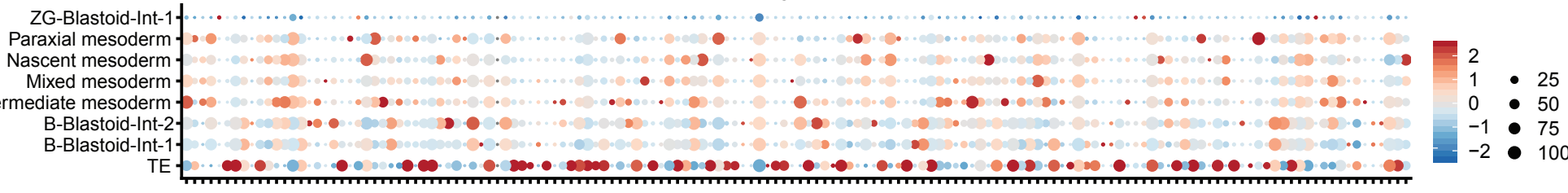


E4.5 chimeras with mScarlet-NLS cells in different culture conditions

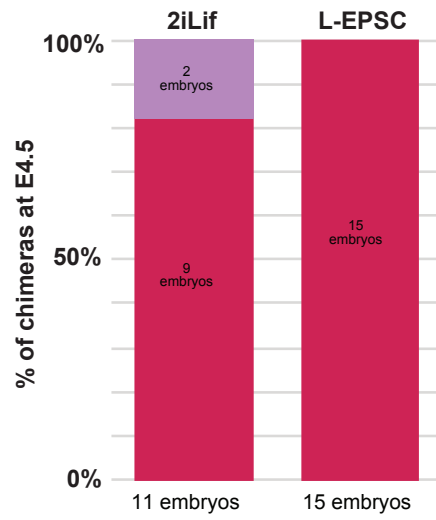

$\mathrm{EPI}+$

TB position

EP
E4.5 chimera with mScarlet-NLS L-EPSCs

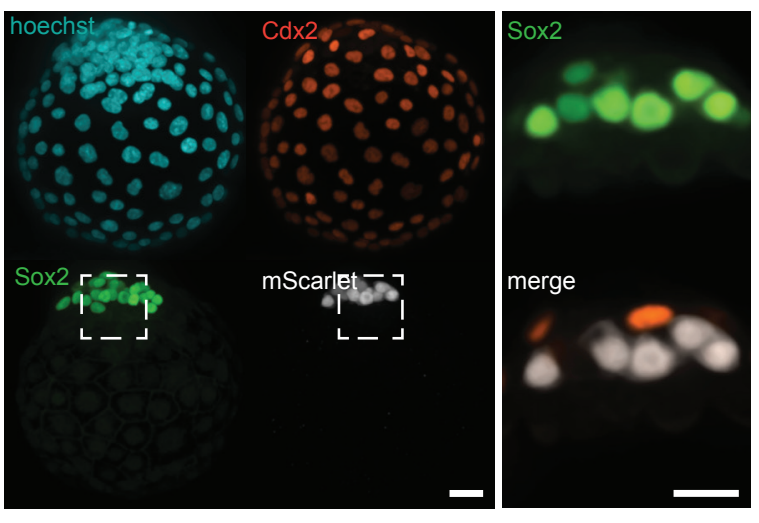

f E6.25 chimera with mScarlet-NLS L-EPSCs

E6.25 chimeras with mScarlet-NLS cells in different culture conditions

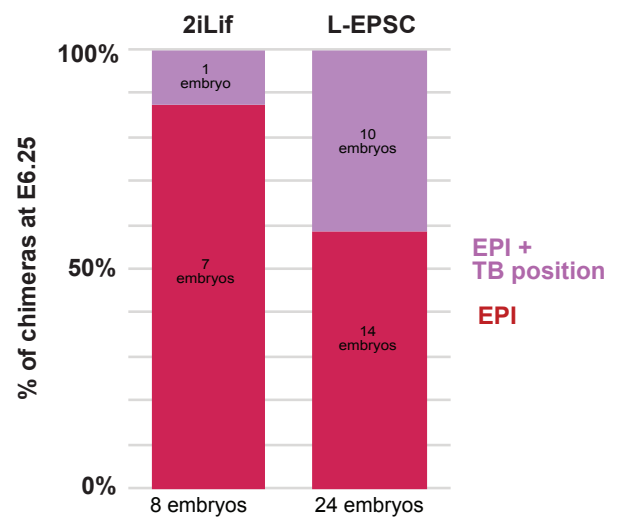

E4.5 chimeras with tdTomato cells in D-EPSC conditions

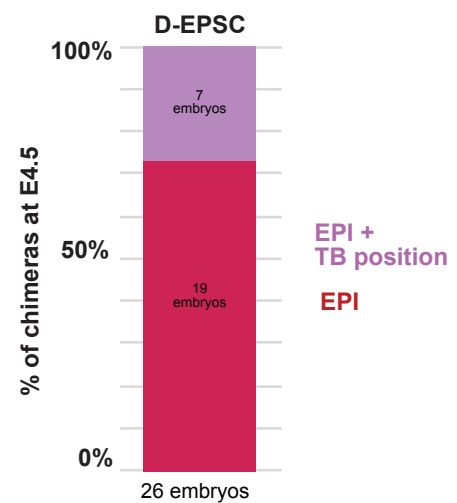

E6.25 chimeras with tdTomato cells in different culture conditions

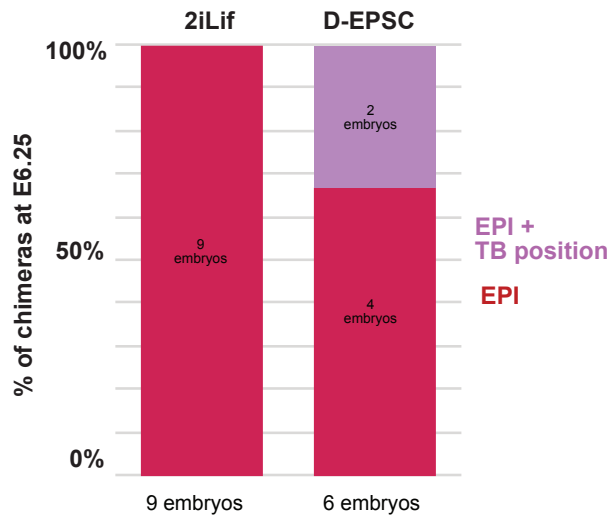

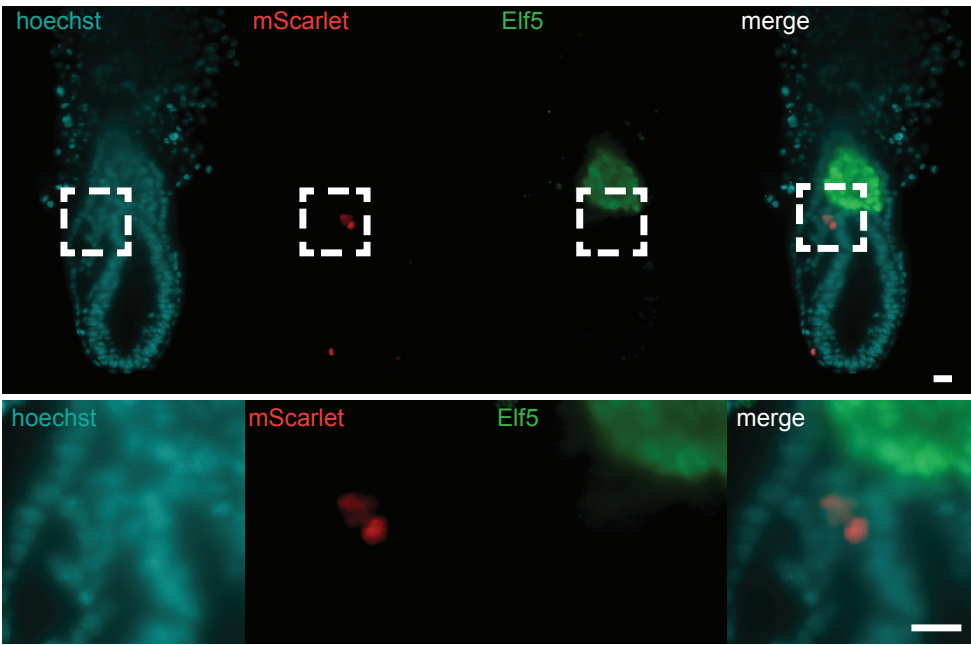

g

E4.5 chimera with tdTomato D-EPSCs
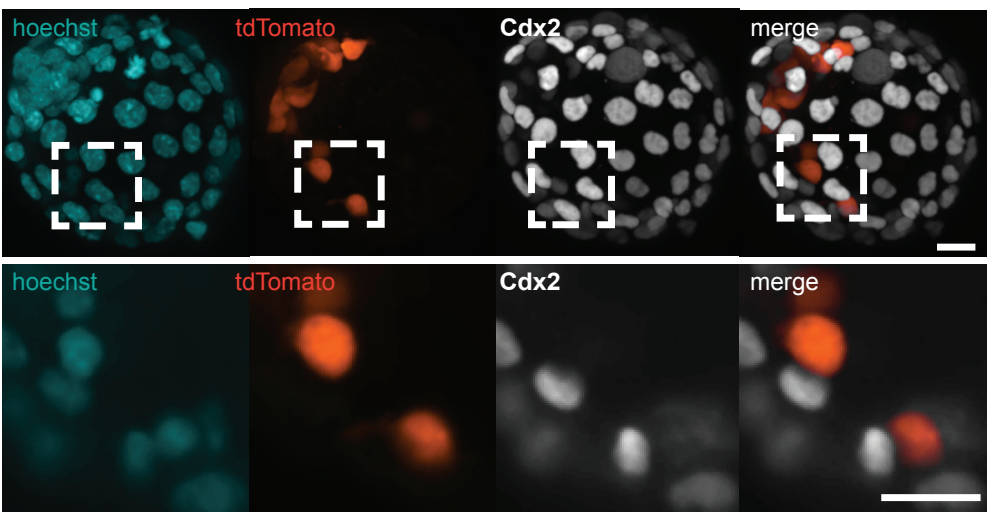

E6.25 chimera with tdTomato D-EPSCs
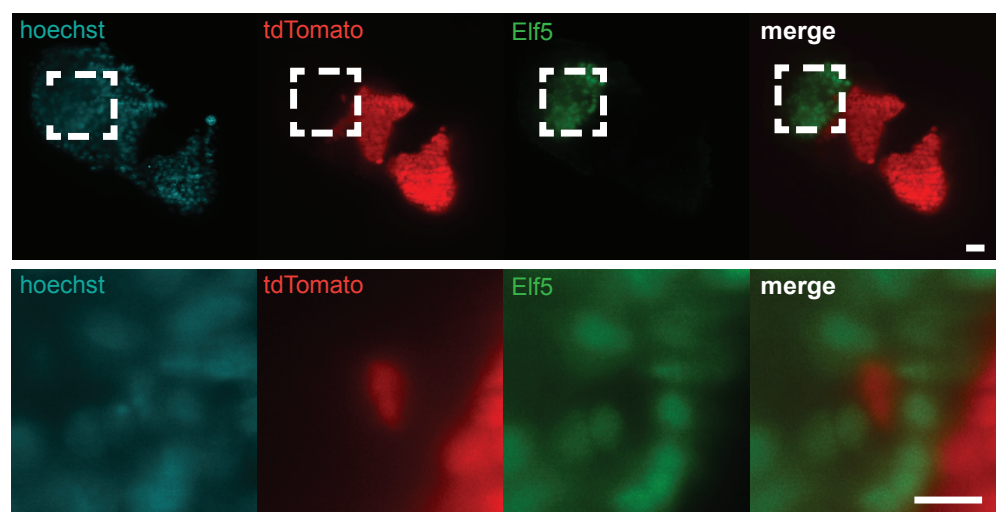

H2B-GFP tetraploid host embryo + WT ESCs

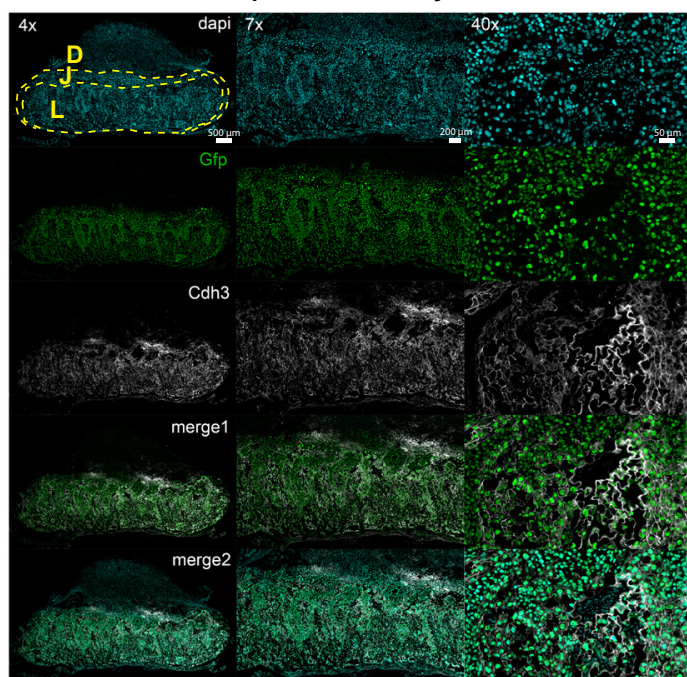

H2B-GFP tetraploid host embryo + WT ESCs

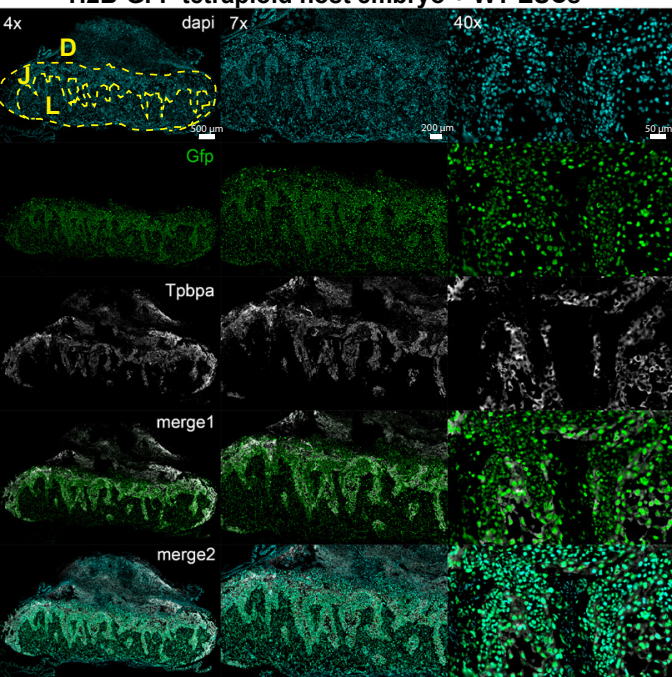

H2B-GFP tetraploid host embryo + WT ESCs

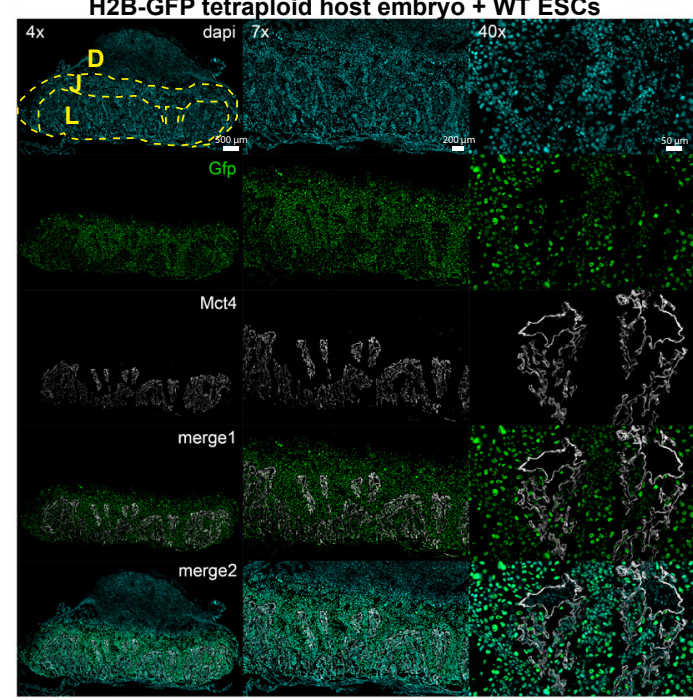

wild-type tetraploid host embryo + H2B-GFP ESCs

wild-type tetraploid host embryo + H2B-GFP L-EPSCs

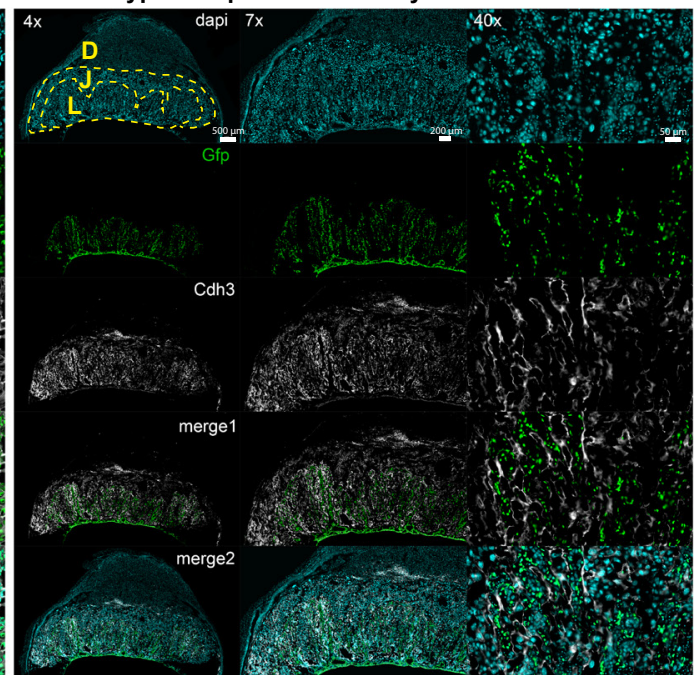

wild-type tetraploid host embryo $+\mathrm{H} 2 \mathrm{~B}$-GFP ESCs

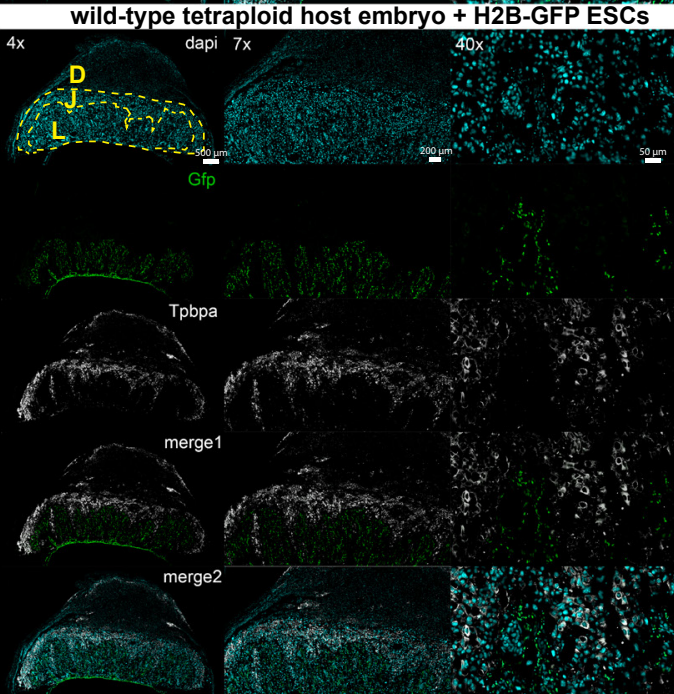

wild-type tetraploid host embryo + H2B-GFP ESCs

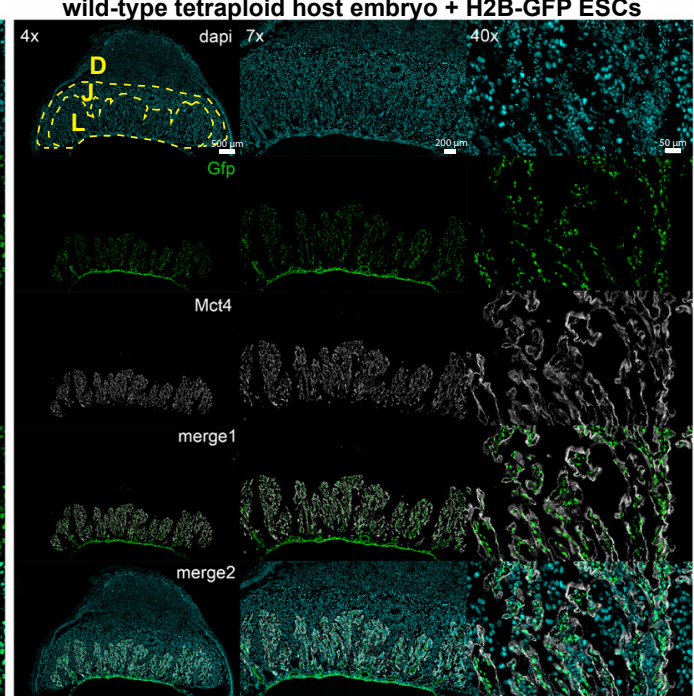

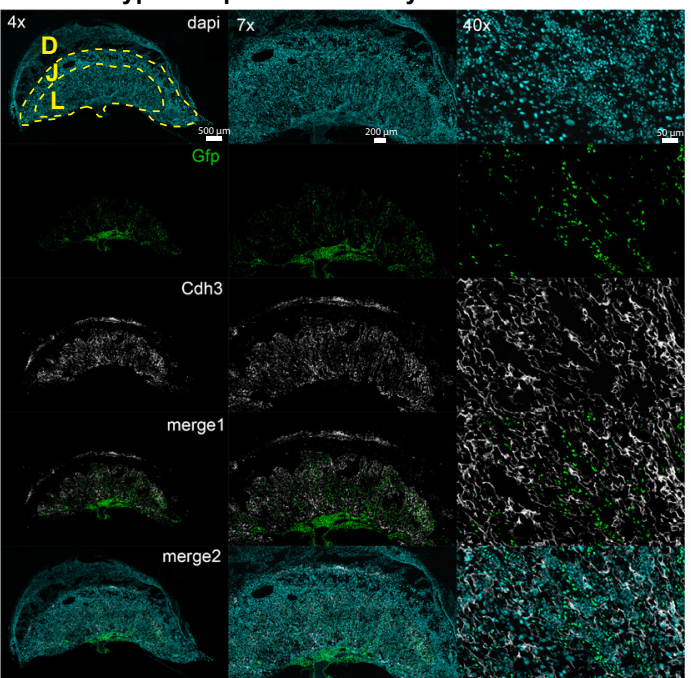

wild-type tetraploid host embryo + H2B-GFP L-EPSCs

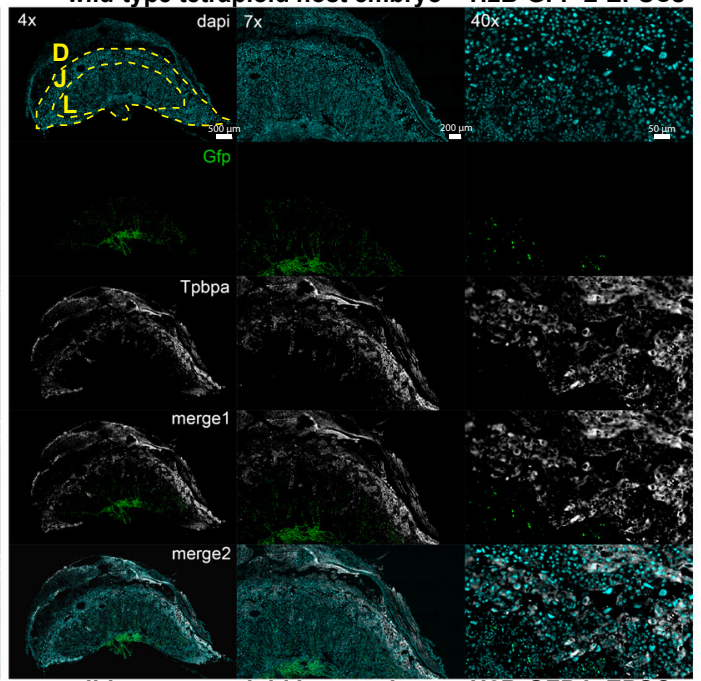

wild-type tetraploid host embryo + H2B-GFP L-EPSCs

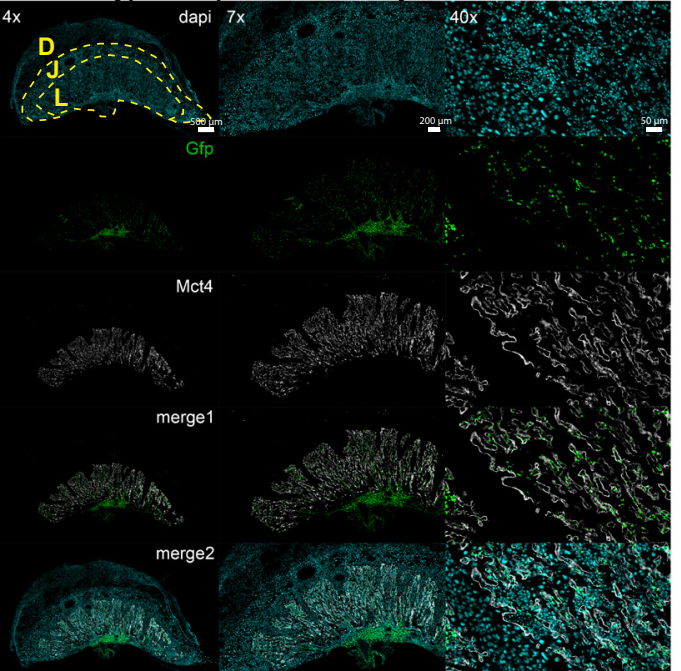


B.Blastoid TE 
B.Blastoid.Int.2 
B.Blastoid PE 
B.Blastoid.Int.1 
B.Blastoid EPI 
Primitive Streak 
EPI E6.5-E7.5 
Visceral endoderm 
ExE ectoderm 
ExE endoderm 
Rostral neurectoderm 
Mixed mesoderm 
ExE mesoderm 
Intermediate mesoderm 
Caudal epiblast 
PGC 
Mesenchyme 
Paraxial mesoderm 
Caudal neurectoderm 
Notochord 
Somitic mesoderm 
Caudal Mesoderm 
Nascent mesoderm 
Parietal endoderm 
Allantois 
Anterior Primitive Streak 
Endothelium 
EPI E5.5 
ICM 
EPI E4.5 
TE

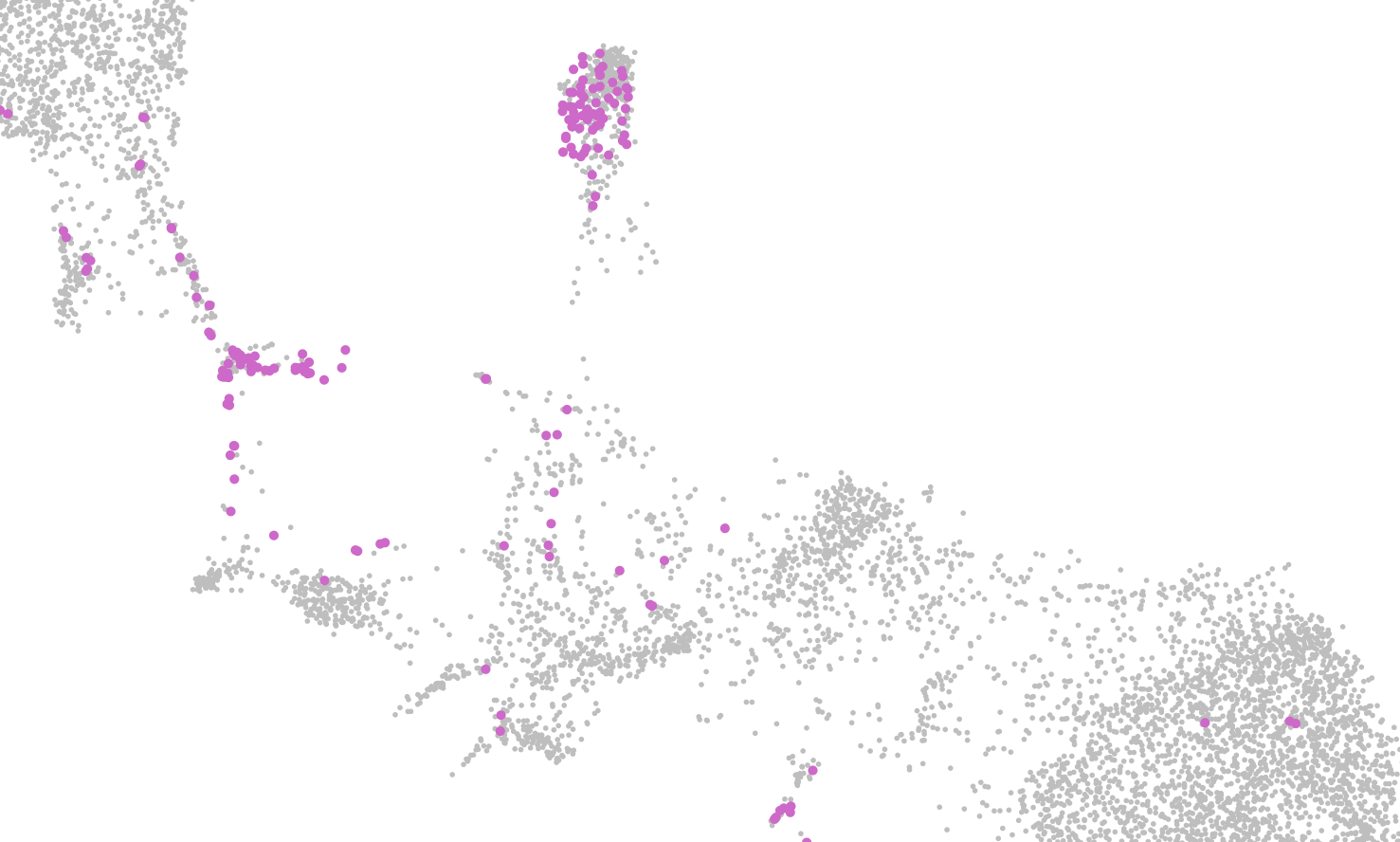


Morula 
PE 
Primed

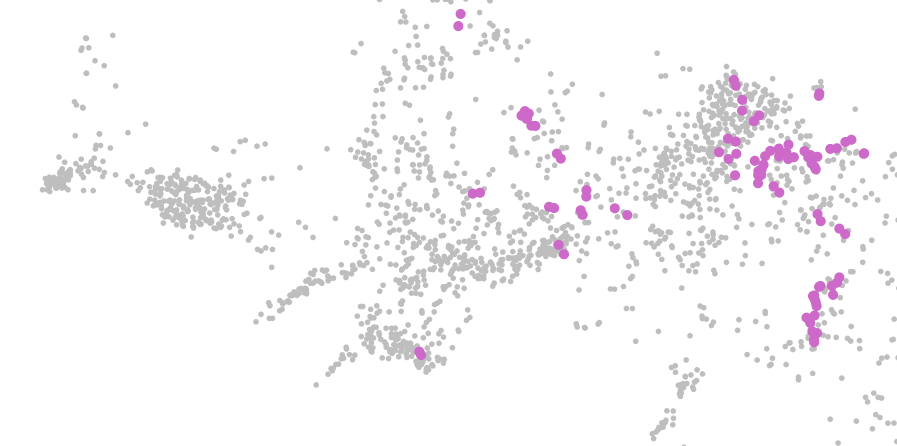


$2 i$ 
ZG.Blastoid.PE 
ZG.Blastoid.TE 
ZG.Blastoid.EPI 
ZG.Blastoid.Int.1 
Supplementary Fig. 1:

UMAP plots generated based on the activity of gene regulatory networks highlighting all individual cell types separately related to SCENIC analysis in Extended data 5. 